\title{
On the mechanisms governing plasma membrane organization - a STED-FCS investigation
}

\author{
Dissertation \\ for the award of the degree \\ "Doctor rerum naturalium" \\ Division of Mathematics and Natural Sciences \\ of the Georg-August-Universität Göttingen
}

submitted by

Débora Machado Andrade

from Aracaju, Brazil

Göttingen 2013 
Prof. Dr. Stefan W. Hell (Reviewer)

Department of Nanobiophotonics

Max Planck Institute for Biophysical Chemistry

Prof. Dr. Jörg Enderlein (Reviewer)

Georg-August-Universität Göttingen

Prof. Dr. Erwin Neher

Membrane Biophysics Department

Max Planck Institute for Biophysical Chemistry

Date of the oral examination: 06.01.2014 


\section{Declaration}

I hereby ensure that the presented thesis "On the mechanisms governing plasma membrane organization - a STED-FCS investigation" has been written independently and with no other sources and aids than quoted.

Göttingen, November $27^{\text {th }} 2013$ 


\begin{abstract}
The plasma membrane is the barrier which localizes and separates life's processes from their external environment. Long described as a free standing two-dimensional fluid mosaic, the organization of the plasma membrane remains elusive. Currently, the most prominent models proposed for its organization lack a consistent body of evidence for their support. Here, we present the first validation of one of these hypotheses: the picket fence model. Using the STED-FCS technique, we demonstrate that cortical actin networks compartmentalize the diffusion of lipids in the plasma membrane of cells. Interestingly, our results further indicate that the Arp $2 / 3$ complex is a major regulator of the cortical actin networks, since inhibiting Arp2/3 leads to specific suppression of compartmentalized diffusion of lipids. Furthermore, the extrusion of the actin cortex promoted by the introduction of myelin-basic protein networks into cells causes lipid diffusion to be modulated by these networks instead. Conclusively, we have elucidated one of the pivotal mechanisms governing the "fluidity" of the plasma membrane mosaic.
\end{abstract}

\title{
Zusammenfassung
}

Die Plasmamembran ist die Barriere, welche die fundamentalen Prozesse des Lebens lokalisiert und von ihrer externen Umgebung begrenzt. Obwohl die Plasmamembran lange Zeit als unabhängiges, zweidimensionales und flüssiges Mosaik beschrieben worden ist, bleibt ihre tatsächliche Organisation unbekannt. Den derzeit gängigen Modellen zur Beschreibung der Plasmamembranorganisation fehlt eine ausreichend experimentelle Basis. In dieser Arbeit präsentieren wir zum ersten Mal eine tatsächliche Verifikation eines dieser Modelle, nämliche des "picket fence models". Mit Hilfe der STED-FCS-Technik demonstrieren wir, dass kortische Aktinnetzwerke die Diffusion von Lipiden in der Plasmamembran von Zellen kompartmentalisieren. Unsere Versuche deuten darauf hin, dass der Arp2/3-Komplex ein Hauptregulator des kortischen Aktinnetzwerks ist, da eine Inhibition von Arp2/3 zu einer spezifischen Unterdrückung der kompartmentalisierten Lipiddiffusion führt. Zusätzlich bewirkt eine Aufösung des kortischen Aktinnetzwerks mittels der Einführung eines Myelin-Basischen Proteinnetzwerks in die Zelle, dass die Diffusion stattdessen durch dieses neue Netzwerk moduliert wird. Zusammenfassend haben wir einen der zentralen Mechanismen hinter der "Fluidität" des Plasmamembran-Mosaiks untersucht und aufgeklärt. 
Para meus pais, Ivana e Augusto

E para meus avós, Maria, Célia, Antônio e Augusto 
"E a coisa mais certa de todas as coisas

Não vale um caminho sob o sol

E o sol sobre a estrada, é o sol sobre a estrada, é o sol"

C. Veloso 


\section{Contents}

Glossary $\quad$ xi

1 Introduction 1

1.1 Unravelling the plasma membrane of cells . . . . . . . . . . . . 1

1.1.1 A brief history of plasma membrane structure . . . . . . . . . . 1

1.1.2 Plasma membrane dynamics and heterogeneity . . . . . . . . 4

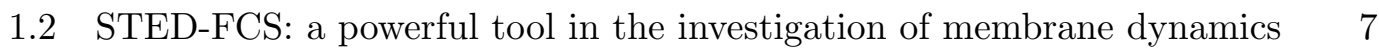

1.3 Aims of this dissertation . . . . . . . . . . . . . . . 11

2 Materials and methods $\quad 13$

2.1 STED-FCS . . . . . . . . . . . . . . . . . 13

2.1 .1 STED-FCS setup . . . . . . . . . . . . . . 13

2.1.2 Calibration of the STED-FCS nanoscope . . . . . . . . 15

2.1.3 STED-FCS cellular measurements . . . . . . . . . . . 16

2.1.4 STED-FCS analysis . . . . . . . . . . . . . . . 18

2.2 Sample preparation . . . . . . . . . . . . . . . . . . 21

2.2.1 Cell culture and transfection . . . . . . . . . . . . . 21

2.2 .2 Labelling procedures . . . . . . . . . . . . . . . . . . . . . . . . . . . . . . . . .

2.2 .3 Drug treatments . . . . . . . . . . . . . . . . . 22

2.3 Monte Carlo simulations for STED-FCS measurements . . . . . . . . . . 23

3 Lipid compartmentalization at the plasma membrane $\quad 25$

3.1 Lipid compartmentalization hypothesis . . . . . . . . . . . 2 25

3.2 Detecting lipid compartmentalized diffusion via STED-FCS . . . . . . 26

3.3 Investigation of molecular mechanisms underlying lipid compartmental-

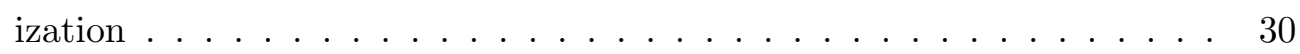

3.3.1 Cytoskeleton modulation significantly impacts lipid compartmentalized diffusion . . . . . . . . . . . . . . . . 31

3.3.2 Cholesterol depletion and myosin II inhibition do not affect lipid compartmentalized diffusion . . . . . . . . . . . . . . 34 
3.4 Discussion . . . . . . . . . . . . . . . . . . . . 34

4 Transient trapping of plasma membrane molecules $\quad 39$

4.1 Lipid diffusion in myelin sheath . . . . . . . . . . . . . . 40

4.1.1 Myelin membrane sheets: a unique biological membrane . . . . . 40

4.1.2 Myelin basic protein modulates lipid diffusion in myelin membrane sheets . . . . . . . . . . . . . . . . 42

4.1 .3 Discussion . . . . . . . . . . . . . . . . . . . 43

4.2 Nanodomain assembly of Ras proteins . . . . . . . . . . . . . . 45

4.3 What is trapping? - Nanodomains and the diffusion law . . . . . . . . 47

5 Conclusions and outlook $\quad 51$

5.1 Plasma membrane dynamics: perspectives for future research . . . . . . 53

$\begin{array}{ll}\text { References } & 55\end{array}$

$\begin{array}{lr}\text { Appendix A } & 63\end{array}$ 


\section{Glossary}

$D_{\text {app }} \quad$ apparent diffusion coefficient

FCS Fluorescence Correlation Spectroscopy

FRAP Fluorescence Recovery After Photobleaching

FWHM full width at half maximum

GalCer galactosylceramide

MBP myelin basic protein

SLBs $\quad$ supported lipid bilayers

SM sphingomyelin

SPT Single-Particle Tracking

STED Stimulated Emission Depletion

STED-FCS Stimulated Emission Depletion Fluorescence Correlation Spectroscopy 


\section{1}

\section{Introduction}

\subsection{Unravelling the plasma membrane of cells}

The plasma membrane of cells plays a main role in pivotal processes related to cell function and maintenance of life itself, such as selective permeability, chemotaxis and plasma membrane protein trafficking. Furthermore, plasma membrane dynamics are at the core of cellular signal transduction pathways. The complexity and specificity with which this structure enables this fundamental process has attracted increasing attention to the investigation of its dynamical organization in the last decades. Despite about ninety years of intense research, many of the mechanisms underlying plasma membrane organization and function still remain elusive. Many proposed models which initially enjoyed great acceptance and successfully explained experimental findings related to the plasma membrane have been eventually proved wrong or incomplete. Currently, the most accepted membrane-organizing principles are still highly debatable, indicating that the state of the art of this field is possibly still scratching the surface of the organization of a highly complex system. Its investigation constitutes one of the most fascinating scientific challenges of modern cell biology.

\subsubsection{A brief history of plasma membrane structure}

The plasma membrane of cells was first reported to be formed by a double bilayer of lipids by E. Gorter and F. Grendel in $1925^{1}$. They postulated that the amphiphilic nature of lipids requires a bilayer structure in order to be stabilized in membranes in an aqueous environment. In this construct, in order to keep the lipids' hydrophobic tails away from the water that is both inside and outside of the cells, the lipids must be arranged so that the polar groups form the outside of such a bilayer, with the tails protected in the middle (Fig. 1.1 A). In order to test this hypothesis, they performed a very elegant experiment aiming to quantify the amount of lipids per area in the plasma 


\section{INTRODUCTION}

membrane. They extracted lipids from the plasma membrane of mammalian red blood cells with acetone, and compared the area occupied by a monolayer formed by the extracted lipids with the total area of red blood cells used in the experiment. The measured ratio between the correspondent lipid monolayer and the total cell surface area was precisely 2:1. Although Gorter and Grendel's estimation of amount of extracted lipids and calculation of the surface area of eritrocytes was later reported to be inaccurate to some extent ${ }^{2}$, this experiment set the foundations for intense subsequent research in this field, and furthermore became a remarkable example of the power of biochemical first principles applied to biology.

Further development in the theory of plasma membrane structure came in 1935, when J.F. Danielli and H. Davson incorporated new findings on membrane tension and plasma membrane protein content, as well as the concept of membrane permeability and pore theory, into the lipid bilayer model proposed by Gorter and Grendel. As a result, a new concept of the plasma membrane structure emerged ${ }^{3}$ : Danielli and Davson proposed that the plasma membrane comprised a lipid bilayer onto which a monolayer of globular proteins was adsorbed onto both sides (Fig.1.1 B). In the membrane model proposed by Danielli and Davson, membrane proteins were assumed to be globular due to the then recent discovery of the pepsin globular structure by J.D. Bernal and D. Crowfoot $^{4}{ }^{\text {a }}$

The advent of electron microscopy set a milestone in membrane research: for the first time, biological membranes could be imaged directly. In the late 1950's, a new paradigm for the organization of biological membranes was set by J.D. Robertson, a pioneering electron microscopist ${ }^{5}$. Besides allowing the visualization of the plasma membrane itself, Robertson's micrographs showed that many other structures inside the cell were also enclosed by membranes. His images showed the structures of all membranes in the cell to be inherently similar, and this unique nature of biological membranes led him to put forward his concept of the "unit membrane". In fact, his model implied more than the ubiquity of biological membranes. With a very bold and non-orthodox model, Robertson hypothesized that the membrane was continuous within a cell, with the plasma membrane being connected to all other internal membranes. ${ }^{\mathrm{b}}$

Despite its initial popularity, the unit membrane model proposed by Robertson ${ }^{5}$ was soon discarded. New developments in fixation techniques for electron microscopy during the 1960's rendered images which discouraged the hypothesis of a continuum between the plasma membrane and the membranes enclosing organelles. Further knowledge

\footnotetext{
${ }^{\text {a }}$ Those were the very early days of protein structure determination, and in fact pepsin was one of the first proteins to be crystallized. The vast and prominent subsequent work on X-ray crystallography by Crowfoot brought a wealth of knowledge of protein and lipid structures, which eventually rendered her the Nobel Prize in Chemistry in 1964.

b Otherwise, Robertson's unit membrane model kept the main features of Danielli and Davson's model, i.e., the plasma membrane was assumed to be formed by a monolayer of proteins adsorbed onto both sides of a lipid bilayer.
} 
A

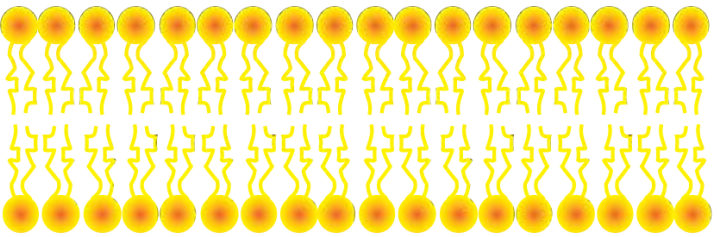

B

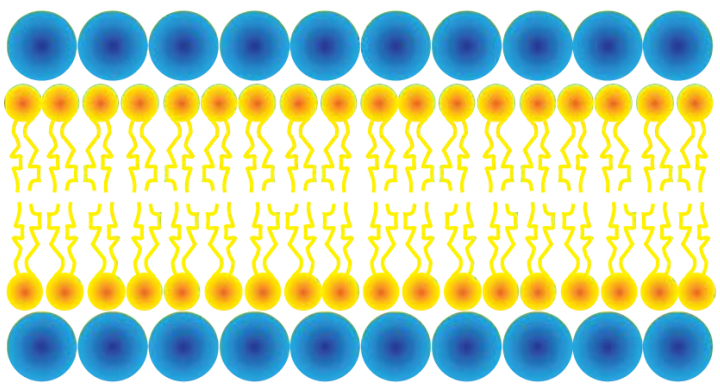

C

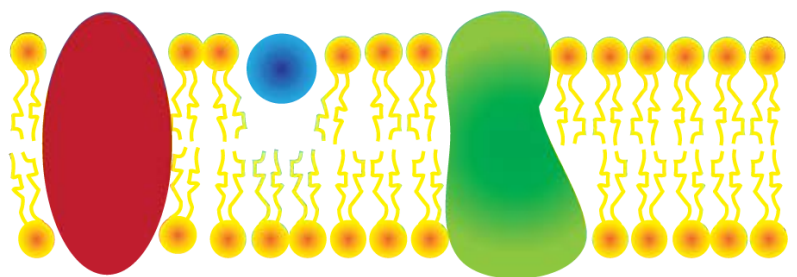

Figure 1.1: Three models for the architecture of the plasma membrane - (A) The lipid bilayer structure originally proposed by Gorter and Grendel. (B) The DanielliDavson model, featuring a layer of globular proteins adsorbed onto both sides of the lipid bilayer. (C) The Singer-Nicolson model (fluid mosaic model), featuring both peripheral and integral proteins embedded in the lipid bilayer. 


\section{INTRODUCTION}

about the plasma membrane and its constituents emerged from a wealth of studies based on electron microscopy and biochemical techniques, and was unified by S.J. Singer and G.L. Nicolson in 1972 in a new model for the plasma membrane architecture, called the "fluid mosaic" model ${ }^{6}$. This model defines the plasma membrane as a mosaic whose matrix is the lipid bilayer, where proteins of different kinds are embedded (Fig.1.1 C). In this model, lipids and proteins are not static, but are assumed to diffuse, hence the "fluidity" of the fluid mosaic model.

Many aspects contribute to the substantial novelty introduced by the fluid mosaic model in the field of membrane research. By discarding the hypothesis of two protein layers sandwiching a lipid bilayer and postulating instead that proteins are embedded in the lipid bilayer, this model reconciled the protein content in biological membranes with its thickness as determined by electron microscopy (between 7.5 and $9.0 \mathrm{~nm}$, according to the images then available). In addition, biological membranes were for the first time considered to be a dynamic system, with all membrane constituents postulated to undergo Brownian diffusion. The diffusion of membrane proteins was first shown in 1970 by L.D. Frye and M. Edidin, who used immunofluorescence to demonstrate that after fusion of a mouse and a human cell, their specific membrane proteins were gradually intermixed ${ }^{7}$. This experiment is one of the pillars in the foundation of the fluid mosaic model. The ubiquitous dynamical character of all membrane constituents was suggested to be connected to fundamental processes of membrane function. Furthermore, for the first time, this model accounted for the thermodynamic stability of membrane proteins and lipids, in which proteins were considered to be largely heterogeneous in molecular weight and form of attachment to the lipid bilayer ${ }^{6}$.

\subsubsection{Plasma membrane dynamics and heterogeneity}

The discovery that plasma membrane molecules are not static, but in fact undergo perpetual diffusion, initiated a whole new field of research. Importantly, the plasma membrane of cells is responsible for controlling the traffic of molecules into and out of the cell, as well as being the ultimate interface where cell signalling takes place. In fact, the first work on signal transduction ${ }^{8}$ coincided in time with the first work on membrane dynamics ${ }^{7}$, both dating from the early 70 's. The dynamics of membrane constituents, assumed to play a fundamental role in membrane function, emerged then as an important phenomenon whose understanding could bring a wealth of knowledge to numerous fields of cell biology.

The first theoretical framework for the diffusion of membrane constituents came in 1975, when P.G. Saffman and M. Delbrück considered the Brownian diffusion equations proposed by A. Einstein ${ }^{9}$ under the boundary conditions imposed by the plasma membrane structure ${ }^{10}$ (as defined by the fluid mosaic model). Saffman and Delbrück concluded that diffusion in a highly viscous two-dimensional medium (the lipid bilayer) 
surrounded by a low viscous medium (water) promotes diffusion that only weakly (logarithmically) depends on the radius of the molecule. A corollary from this theory is that proteins would be expected to diffuse with approximately the same diffusion coefficient, regardless of their size, and further that oligomerization of proteins would not impact diffusion significantly.

Numerous biophysical techniques using fluorescent probes or gold particles were conceived in the following years, pursuing the investigation of dynamics and interactions of cellular molecules. Fluorescence Correlation Spectroscopy (FCS) ${ }^{11}$, Fluorescence Recovery After Photobleaching (FRAP) ${ }^{12}$, Single-Particle Tracking (SPT) ${ }^{13,14}$ and optical tweezers ${ }^{15}$ are examples of these microscopy-based techniques that would later change the paradigms of the fluid mosaic model. In particular, studies based on FRAP, SPT and optical tweezers in the 80s and 90s rendered inaccurate the notion that proteins undergo Brownian diffusion in the plasma membrane ${ }^{15-17}$, which was one of the main assumptions of the fluid mosaic model ${ }^{6}$. Instead, diffusion of proteins was observed to be highly heterogeneous.

Gradually, the heterogeneity of lipid distribution throughout the plasma membrane also became a more plausible hypothesis than the homogeneous lipid bilayer postulated by the fluid mosaic model. The first evidence for lipid segregation came from the discovery that different types of lipids partition differently into the outer and inner leaflets of the double bilayer of the plasma membrane ${ }^{18}$. Furthermore, epithelial cells were found to be polarized, i.e. their apical membrane features different lipid composition from the basal membrane ${ }^{19}$. In 1997, K. Simons and E. Ikonen put those findings in a broader context of lipid self-assembly, putting forward a new construct for plasma membrane organization, whose underlying principle is the self-association of specific lipids into stable platforms ${ }^{20}$. They coined the term "lipid rafts" to denote these platforms, postulated to be regions of the plasma membrane highly enriched in sphingolipids, cholesterol and lipid-anchored proteins. In lipid rafts, lipids are organized in liquid ordered phase $\left(\mathrm{L}_{o}\right)$, which is characterized by a high degree of acyl-chain ordering $^{21}$. Conversely, membrane lipids out of rafts are organized in liquid disordered phase $\left(\mathrm{L}_{d}\right)$. According to the model introduced by Simons and Ikonen, lipid rafts are assumed to have specific protein selectivity, increasing the probability of interaction of non-abundant proteins and receptors, with lipid self-assembly being the driving force for heterogeneity of distribution of membrane molecules.

Because the lipid rafts hypothesis could successfully describe a potential mechanism for the most important cell membrane functions by proposing an elegant model for membrane sorting, it has been enjoying vast acceptance by the scientific community since its formulation, becoming one of the most important paradigms of modern cell biology ${ }^{22}$. In fact, functions attributed to lipid rafts have spanned the manifold functions ascribed to the plasma membrane, with several connections between these 


\section{INTRODUCTION}

lipid platforms and diseases been postulated ${ }^{23,24}$. Nevertheless, the very existence of lipid rafts is still debatable ${ }^{23,25}$. The foundations for the lipid rafts hypothesis relied on experiments with detergent extraction of biological membranes, whose conclusions were drawn into question by several studies ${ }^{25}$. Although recent advances in biophysical techniques have later allowed a more compelling body of evidence ${ }^{21,22}$, more conclusive proofs, such as the direct visualization of lipid rafts, as well as the determination of their characteristic lifetime, are still missing.

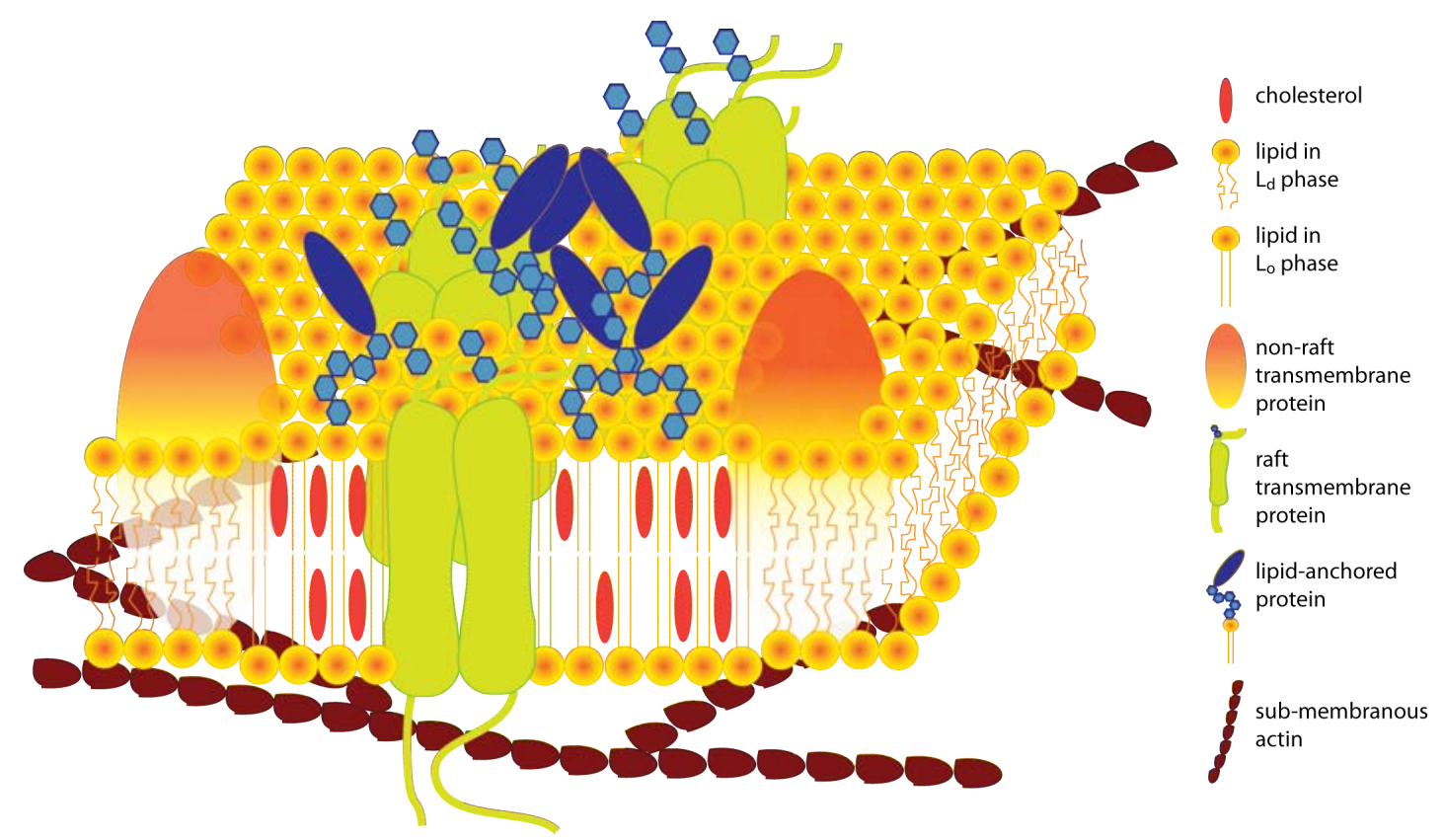

Figure 1.2: Combination of the two currently most prominent membraneorganizing principles: lipid rafts and pickets and fences - Lipids in the raft phase are in the liquid-ordered phase, whereas lipids out of the raft are in the liquid-disordered phase. Different types of membrane proteins are postulated to have different affinities to lipid rafts. The sub-membranous cortical actin partially compartmentalize proteins and lipids, including rafts.

In addition to the lipid rafts hypothesis, yet another major organizing principle has been put forward to explain the dynamics of membrane molecules: the picket fence model, proposed by A. Kusumi ${ }^{26}$. This model hypothesizes that the sub-membranous actin cortex hinders the diffusion of transmembrane proteins, which tend to anchor to the underlying actin filaments. These transmembrane proteins in turn constrain the diffusion of other proteins and lipids, effectively compartmentalizing the plasma membrane. This model was proposed in view of SPT studies on membrane receptors ${ }^{16}$ and lipids ${ }^{27}$, which revealed compartmentalized diffusion on the surface of the plasma membrane. Kusumi coined the term "hop diffusion" to denote this diffusion pattern. Compartmentalization of membrane constituents was postulated to be implicated, for 
example, in localized signalling ${ }^{28}$. However, this model too remains controversial ${ }^{29}$. While the notion of protein diffusion being constrained by the actin cytoskeleton seems to be indisputable ${ }^{30}$, all supporting evidence for that lipids undergo compartmentalized diffusion was so far provided exclusively by SPT experiments, in which artefact-prone probes were used ${ }^{31}$.

Currently, lipid rafts and pickets fence are the most prominent models proposed for the organization of the plasma membrane. These models are not mutually exclusive (Fig. 1.2), and in fact can be unified in a hierarchical scheme that confers different organizing principles to the different spatial scales under consideration ${ }^{32}$. Furthermore, yet other mechanisms of molecular organization at the plasma membrane have been proposed. One such proposal suggested that nanoclusters of lipid-anchored proteins and signalling proteins are first assembled via interaction with dynamic actin filaments dissociated from the actin cytoskeleton mesh and free to diffuse throughout the membrane cortex $^{33}$. This sorting mechanism would facilitate nanocluster assembling of membrane proteins by using the same strategy which is attributed to lipid rafts: preferential interactions of proteins to specific molecules increase their probability of interaction among themselves, thereby facilitating nanoclustering.

In summary, research carried out in the last decades has revealed the plasma membrane to be a profoundly complex dynamical system. Continuous improvement of biophysical techniques have brought important insights into this field, but more sophisticated methods are yet required to answer the yet unresolved questions. In particular, limitations in spatio-temporal resolution of the available methods and limitations in the available labelling strategies for probing membrane molecules still prevent the solution of the most pressing questions. In the following section, a powerful technique with great potential to shed light on important unsolved problems in membrane dynamics is introduced.

\subsection{STED-FCS: a powerful tool in the investigation of membrane dynamics}

Due to the wave nature of light, conventional fluorescence microscopy is limited in resolution by the diffraction of the converged beam that forms the focal spot. This diffraction limit was first put forward by E.K. Abbe, who calculated the resolution limit of the microscope to be ${ }^{34}$ :

$$
d=\frac{\lambda}{2 \mathrm{NA}}
$$

where $d$ stands for the achievable resolution by a microscope, $\lambda$ is the wavelength of light used for illumination of the sample and $N A$ denotes the numerical aperture of the microscope. 


\section{INTRODUCTION}

Having become a standard law in optical physics, Abbe's equation was reformulated in 1994, when S.W. Hell proposed a new physical concept in fluorescence microscopy which effectively renders this technique diffraction-unlimited: Stimulated Emission Depletion (STED) ${ }^{35}$. In this construct, fluorophores within the diffraction-limited focus become distinguishable by inducing them to transiently assume distinct fluorescent states $^{36,37}$. The excitation beam causes the molecules within the diffraction-limited spot to be sent to a fluorescent state $\left(\mathrm{S}_{1}\right)$. Subsequent STED illumination de-excites a fraction of the molecules, which return to the non-fluorescent ground state $\left(\mathrm{S}_{0}\right)$. Typically, a doughnut-shaped beam is used for inducing this stimulated emission. Thus, the collected fluorescence originates solely from within a central area much smaller than the diffraction limit. The photons emitted by stimulated emission can be separated by appropriate filters from fluorescence, by de-exciting at a wavelength sufficiently larger than the emission peak of the fluorophore (Fig.1.3 A). The effective resolution achievable by this technique depends in principle only on the intensity of the STED beam at the focal plane ${ }^{38}$ :

$$
d_{\mathrm{STED}}=\frac{d_{\mathrm{conf}}}{\sqrt{1+\frac{I_{\mathrm{STED}}}{I_{\mathrm{s}}}}}
$$

where $d_{\text {STED }}$ stands for the resolution achievable by a STED microscope, $d_{\text {conf }}$ is the diffraction-limited resolution of a confocal microscope, exciting at a given wavelength, $I_{\mathrm{STED}}$ is the STED beam intensity at the focal plane and $I_{\mathrm{S}}$ is a saturation intensity, dependent on the fluorophore and on the STED wavelength.

STED microscopy, being the first concept to break the diffraction limit ${ }^{39}$, has significantly broadened the applicability of fluorescence imaging to the life sciences in the last years ${ }^{40-43}$. However, the applications of STED are not limited to imaging. This ingenious concept will find applications whenever overcoming the diffraction limit in fluorescence techniques is necessary or useful. A beautiful example that illustrates this idea is the method of Stimulated Emission Depletion Fluorescence Correlation Spectroscopy (STED-FCS), which emerges as a powerful technique to assess dynamics of membrane molecules on the nanoscale ${ }^{44-46}$. By combining FCS and STED microscopy, this technique is able to resolve membrane dynamics with unprecedented resolution ${ }^{44,47,48}$.

STED-FCS shares the same theoretical principles of FCS. The first realization of FCS occured in 1972, with the seminal work of D. Magde, E. Elson and W.W. Webb ${ }^{11}$. Nonetheless, its underlying probabilistic principles trace back to the development of stochastic theory in the beginning of the 20th century. The following excerpt from the review "Stochastic Problems in Physics and Astronomy" from 1943, by the prominent physicist S. Chandrasekhar, illustrates that ${ }^{49}$ :

"Suppose we observe by means of an ultramicroscope a small well-defined element of 
A

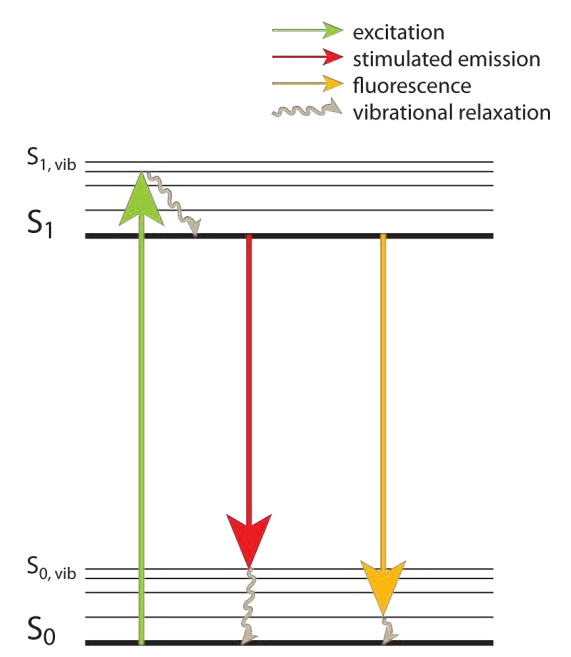

B

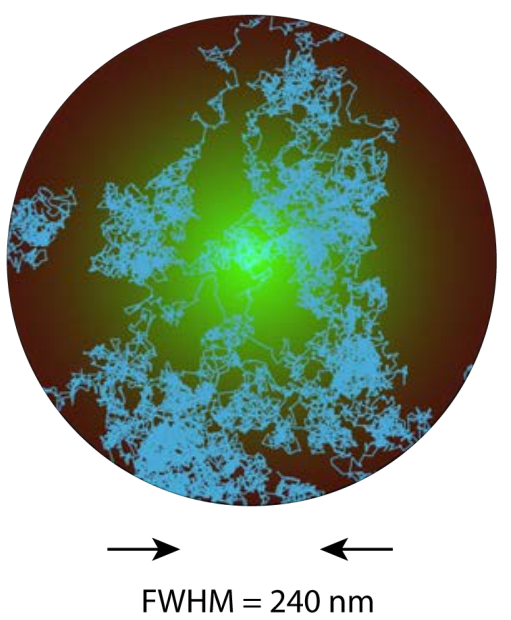

C
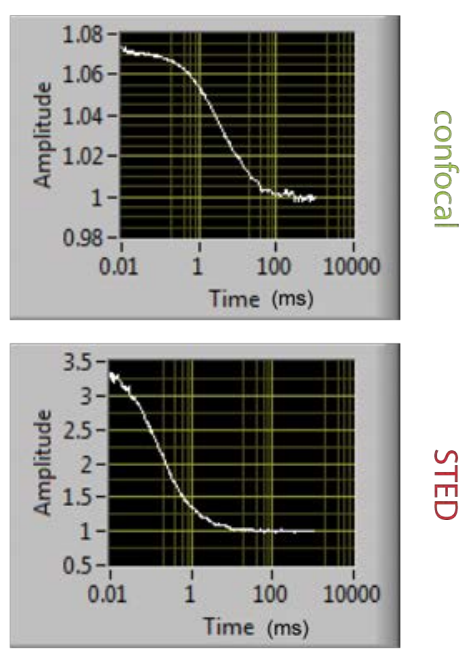

管

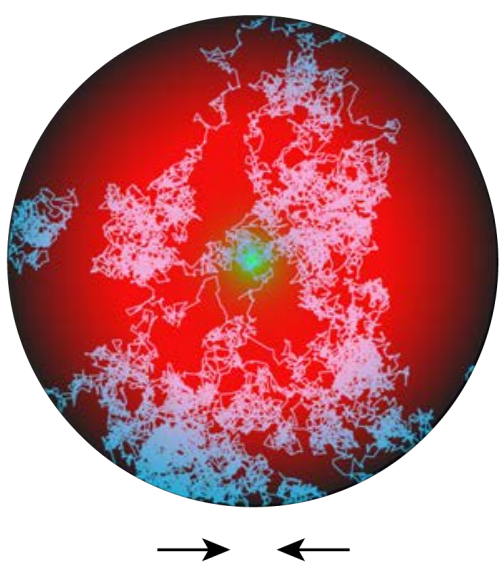

$\mathrm{FWHM}<40 \mathrm{~nm}$

Figure 1.3: STED-FCS assesses lipid dynamics on the nanoscale - (A) Jablonski diagram illustrating the photophysics of stimulated emission depletion. Horizontal lines represent the energy levels of the fluorophore. After excitation to a higher electronic state (e.g. $\mathrm{S}_{1}$ ), fluorescence is a spontaneous transition that brings the fluorophore back to the ground state $\left(\mathrm{S}_{0}\right)$, usually to a low vibrational level. Stimulated emission is typically implemented with light of a longer wavelength, that induces transition to one of the higher vibrational states. In this way, experimental separation between fluorescence and STED beams is achieved by appropriate spectral filters. (B) A doughnut-shaped STED beam (red) shuts down fluorescence in the periphery of the confocal spot area (green), allowing diffraction-unlimited FCS. In the experiments described in this work, the diameter of the observation area, or full width at half maximum (FWHM), varied between $240 \mathrm{~nm}$ down to $<40 \mathrm{~nm}$. (C) Experimental correlation curves for confocal and STED recordings. The amplitude of the autocorrelation function, which is inversely proportional to the temporal average of the number of fluorescent molecules in the focal spot, exemplifies the decrease in the effective observation area for STED recordings. 


\section{INTRODUCTION}

volume of a colloidal solution and count the number of particles in the element at definite intervals of time $\tau, 2 \tau, 3 \tau$, etc., and record them consecutively. We shall further suppose that the interval $\tau$ between successive observations is not large. Then the number which is observed on any particular occasion will be correlated in a definite manner with what was observed on the immediately preceding occasion. This correlation will depend on a variety of physical factors including the viscosity of the medium: thus it is clear from general considerations that the more viscous the surrounding medium the greater will be the correlation in the numbers counted on successive occasions."

Surprisingly, Chandrasekhar's visionary construct defines the basic principles of FCS with great accuracy. Or more precisely, it defines the principles of STED-FCS, which is a diffraction-unlimited technique, by employing an "ultramiscroscope" in its experimental realization, i.e. the STED microscope. In STED-FCS, photon counts stemming from fluorophore-labelled molecules diffusing through the focal spot are correlated in time according to the formula ${ }^{50}$ :

$$
G(\tau)=\frac{\langle\delta F(t) \delta F(t+\tau)\rangle}{\langle F(t)\rangle^{2}}
$$

where $G(\tau)$ is the autocorrelation function, $F(t)$ stands for fluorescence counts at a time $t$ and $\delta F$ stands for fluctuations of the fluorescence counts with respect to the average $\langle F(t)\rangle$. The autocorrelation function is a measure of the self-similarity of the distribution of molecules in the focal volume over the time. This function provides information about physical-chemical processes undergone by the fluorescent molecules in the relevant time scales. For example, characteristic dark state transitions, binding reactions and diffusion rates are typical processes assessed by the analysis of the autocorrelation function $^{51}$.

STED-FCS takes advantage of the improved lateral resolution provided by STED in order to investigate diffusion of molecules on the plasma membrane on the nanoscale. Since the lateral resolution of a STED microscope is correlated to the STED beam intensity at the focal plane (Eq. (1.2)), STED-FCS allows the assessment of diffusion in a tunable, diffraction-unlimited observation area (Fig.1.3 B,C). This represents an exceptional breakthrough for spot-variation FCS, a method that discerns diffusion patterns based on the relation among FCS recordings at different sizes of the observation area $^{52,53}$. In this context, STED-FCS permits the realization of spot-variation FCS in areas comparable in size to the putative lipid rafts and cytoskeleton-based submembranous compartments, supposedly the two major structures responsible for membrane organization. 


\subsection{Aims of this dissertation}

This work aims to elucidate the validity of the picket fence model, which hypothesizes that cortical actin networks form compartments that partially segregate lipids and proteins in the plasma membrane. This model, currently one of the most important organizing principles for the plasma membrane, has been the subject of debate for many years due to the fact that limitations in spatio-temporal resolution of the available methods to probe membrane dynamics have precluded its validation.

In order to achieve this goal, we used STED-FCS, a diffraction-unlimited technique which allows a systematic probing of molecular diffusion with high spatio-temporal resolution. With spatial resolution below $40 \mathrm{~nm}$, STED-FCS is able to resolve lipid diffusion within the putative membrane compartments ${ }^{28,54}$.

This dissertation is organized as follows. In chapter 2 , the methods and materials utilized in this research are detailed. Chapter 3 presents the contentious aspects of the picket fence hypothesis, and shows how we, for the first time, validate that model. Additionally, we determine the underlying cellular mechanisms that allow lipid compartmentalization in the plasma membrane. Chapter 4 introduces the investigation of lipid diffusion in myelin membrane sheets and demonstrate that in this special membrane, the underlying meshwork of myelin basic protein modulates lipid diffusion. Furthermore, nanoclustering of Ras signalling proteins is observed. In chapter 5 the final conclusions of this work are made, and an outlook for further developments is presented. 
1. INTRODUCTION 


\section{2}

\section{Materials and methods}

\section{$2.1 \quad$ STED-FCS}

STED-FCS was the method here utilized to investigate membrane dynamics. The STED-FCS setup and its calibration procedure, as well as details about cellular measurements are described in the following.

\subsubsection{STED-FCS setup}

The STED nanoscope (Fig. 2.1) was based on a home-built confocal microscope setup equipped with a $640 \mathrm{~nm}$ laser ( 100 ps pulse width, LDH-D-C-640, PicoQuant) for excitation of the fluorescent label with a repetition rate of $45 \mathrm{MHz}$. The STED beam was provided by a mode-locked Titanium:Sapphire laser system (Chameleon, Coherent Inc.) operating at $780 \mathrm{~nm}$ with a repetition rate of $90 \mathrm{MHz}$. The time interval between the pulses of both lasers was adjusted using a home-built electronic delay unit, where the STED pulses served as the trigger master. The STED laser pulses were stretched from 200 fs to a pulse length of approximately 180 ps using four $30 \mathrm{~cm}$ optical SF6 glass rods and a 125m-long polarization maintaining single-mode fiber (OZ Optics).

The laser beams were spatially overlaid and the fluorescence light filtered by appropriate (dichroic) filters (AHF Analysentechnik). The doughnut-shaped focal spot of the STED beam featuring a central intensity zero was produced by introducing a phase-modifying plate (RPC Photonics) into the beam path, imprinting on the wave front a helical phase $\operatorname{ramp} \exp (\mathrm{i} \varphi)$ with $0 \leq \varphi \leq 2 \pi$. The STED beam, initially Gaussian shaped, acquires a toroidal conformation after being converged by the objective lens. That is due to the fact that the phase retardation imprinted by the phase plate on the wave front causes destructive interference along the optical axis upon convergence of the beam. A $\lambda / 4$-plate (B. Halle) ensured circular polarization of the STED and excitation beams. Fluorescence excitation and collection was realized using an 


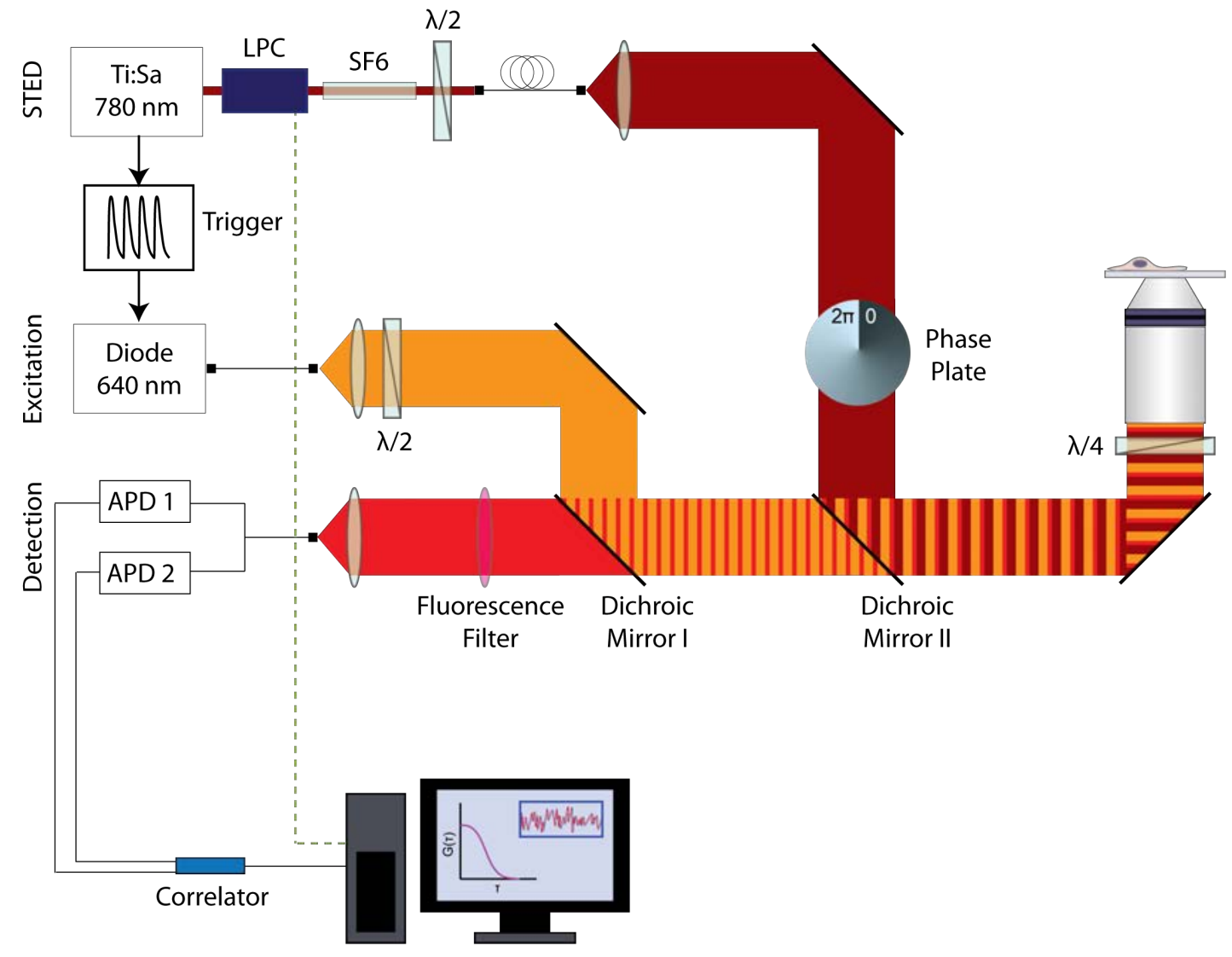

Figure 2.1: Schematics of the STED-FCS setup - The STED nanoscope used in the experiments reported in this thesis comprises one diode laser used for excitation of the fluorescent molecules and a Ti:Sa laser used for STED. Both excitation and STED beams, after being collimated, are combined by appropriate dichroic mirrors. The emitted fluorescence is filtered by a bandpass filter and split onto two avalanche photo diodes (APDs). The cross-correlation between the signals originated by the APDs is done by a correlator module. During the experiment, the STED beam power is varied by a softwarecontrolled laser power control (LPC) (Brockton Electro-Optics). The combination of $\lambda / 2$ and $\lambda / 4$ plates facilitates circular polarization for the excitation and STED beams. 
oil immersion objective (APON 60x, NA $=1.49$, Olympus). A confocal pinhole was provided by coupling the fluorescence onto a multi-mode fiber (Fiber Optic Network Technology). Additionally, this fiber splits the signal 50:50, which was then detected by two single-photon counting modules (avalanche photo diode SPCM-AQR-13-FC, Perkin Elmer Optoelectronics) and the recorded fluorescence counts were further processed by a hardware correlator card (Flex02-01D, Correlator.com). The intensity distribution of the excitation and STED light were measured by scanning a scattering gold bead of $80 \mathrm{~nm}$ in diameter (gold colloid, EMGC80, BBinternational) using a non-confocal detector (MP 963 Photon Counting Module, Perkin Elmer).

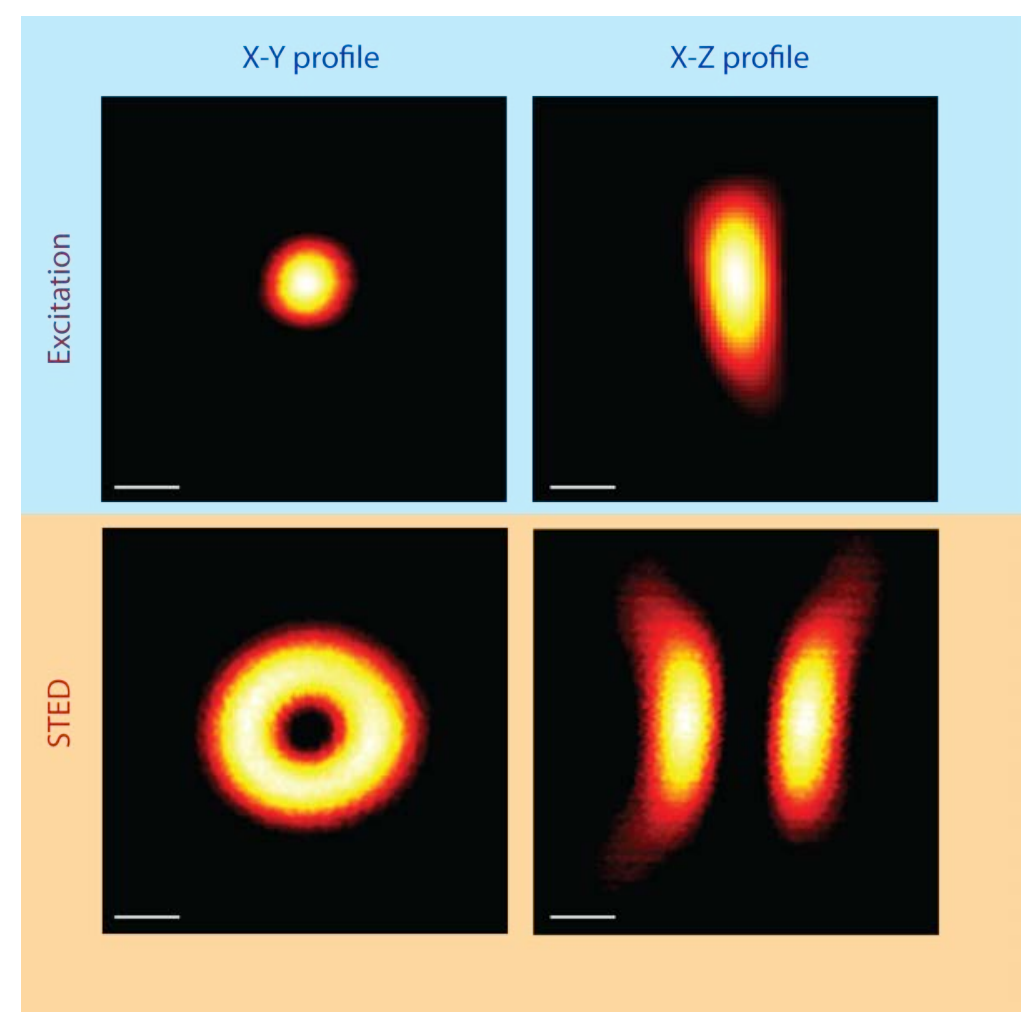

Figure 2.2: Experimental intensity distributions of excitation and STED beams - A colloidal gold particle was used to scatter both excitation and STED beams, allowing the visualization of their intensity distributions. This procedure facilitates the alignment of the STED setup, which consists of spatially overlaying both beams, co-centered, and ensuring the symmetry of their intensity distributions. Scale bars are $250 \mathrm{~nm}$

\subsubsection{Calibration of the STED-FCS nanoscope}

The laser powers $P$ were measured at the back focal plane of the objective. Together with the full width at half maximum (FWHM) of the focal laser intensity distribution, they allow the calculation of the time-averaged intensity $I=P /\left[\pi(F W H M / 2)^{2}\right]$. Cal- 


\section{MATERIALS AND METHODS}

ibration of the diameter $d\left(P_{\mathrm{STED}}\right)$ of the effective focal fluorescence spots formed by a certain STED power $P_{\text {STED }}$ was performed by STED-FCS measurements of fluorescent lipid analogues in supported lipid bilayers (SLBs), for that such lipid bilayers provide a two-dimensional free diffusing system of molecules, labelled with the same fluorophores used in our cellular experiments. The confocal FWHM being determined by fluorescent beads $\left(d\left(P_{\text {STED }}=0\right) \sim 240 \mathrm{~nm}\right)$, the other effective diameters $d\left(P_{\text {STED }} \neq 0\right)$ can be calculated by performing STED-FCS measurements on supported lipid bilayers and using the relation:

$$
\frac{d\left(P_{\mathrm{STED}}=0\right)}{d\left(P_{\mathrm{STED}}\right)}=\sqrt{\frac{t_{D\left(P_{\mathrm{STED}}=0\right)}}{t_{D\left(P_{\mathrm{STED}}\right)}}}
$$

where $t_{D}$ stands for the transient times correspondent to each given $P_{\mathrm{STED}}$. The relation above stems from the fact that the lipids in SLBs undergo two-dimensional free diffusion, so that the diffusion time scales proportionally with the diffusion area.

The SLBs were prepared based on a procedure described in detail elsewhere ${ }^{55}$. The lipid DOPC (1,2-dioleoyl-sn-glycero-3-phosphocholine, Avanti) and the fluorescent lipid analogue (DPPE-Atto647N, Atto-Tec) were mixed in organic solvents (Chloroform/MeOH 1:1) at a lipid concentration of $\sim 1 \mathrm{mg} / \mathrm{ml}$. The ratio of labelled lipids per non-labelled ones was approximately 1:10,000. $50 \mu \mathrm{l}$ of such solution were dropped onto a piranha-cleaned (Femto-RF, Diener Electronic) standard microscope cover glass (diameter $22 \mathrm{~mm}$, no. 1.5 thickness) and spin-coated at $60 \mathrm{~Hz}$ for about one minute. The cover glass was then placed in a microscopy chamber and subsequently the dry thin lipid film was rehydrated with $500 \mu \mathrm{l}$ buffer solution (150 mM NaCl, $10 \mathrm{mM}$ HEPES). Such bilayers were stable for several hours.

\subsubsection{STED-FCS cellular measurements}

We assessed the dynamics of fluorescent labelled molecules by placing the focused cocentered excitation and STED beams on random positions at the plasma membrane. Near the edge of cells, lipid diffusion is probed concurrently in the apical and basal membranes (Fig. 2.3 A). That happens because the effective observation volume is decreased only laterally by the STED beam, remaining approximately $700 \mathrm{~nm}$ long in the axial direction (for excitation wavelength of $640 \mathrm{~nm}$ ), encompassing both membranes when they are close enough. In contrast, in the cell body of NRK cells, the measurements were performed at the basal membrane, since the bulky cell body of these cells features a separation between apical and basal membranes of $\sim 2$ to $7 \mu \mathrm{m}$, which ensures STED-FCS experiments to probe only the basal membrane (Fig. 2.3 B).

Measurements were taken at room temperature and completed before any significant morphological changes in the cell could occur. All measurements were carried out during $10 \mathrm{~s}$, providing a correlation time two orders of magnitude longer than the 
A
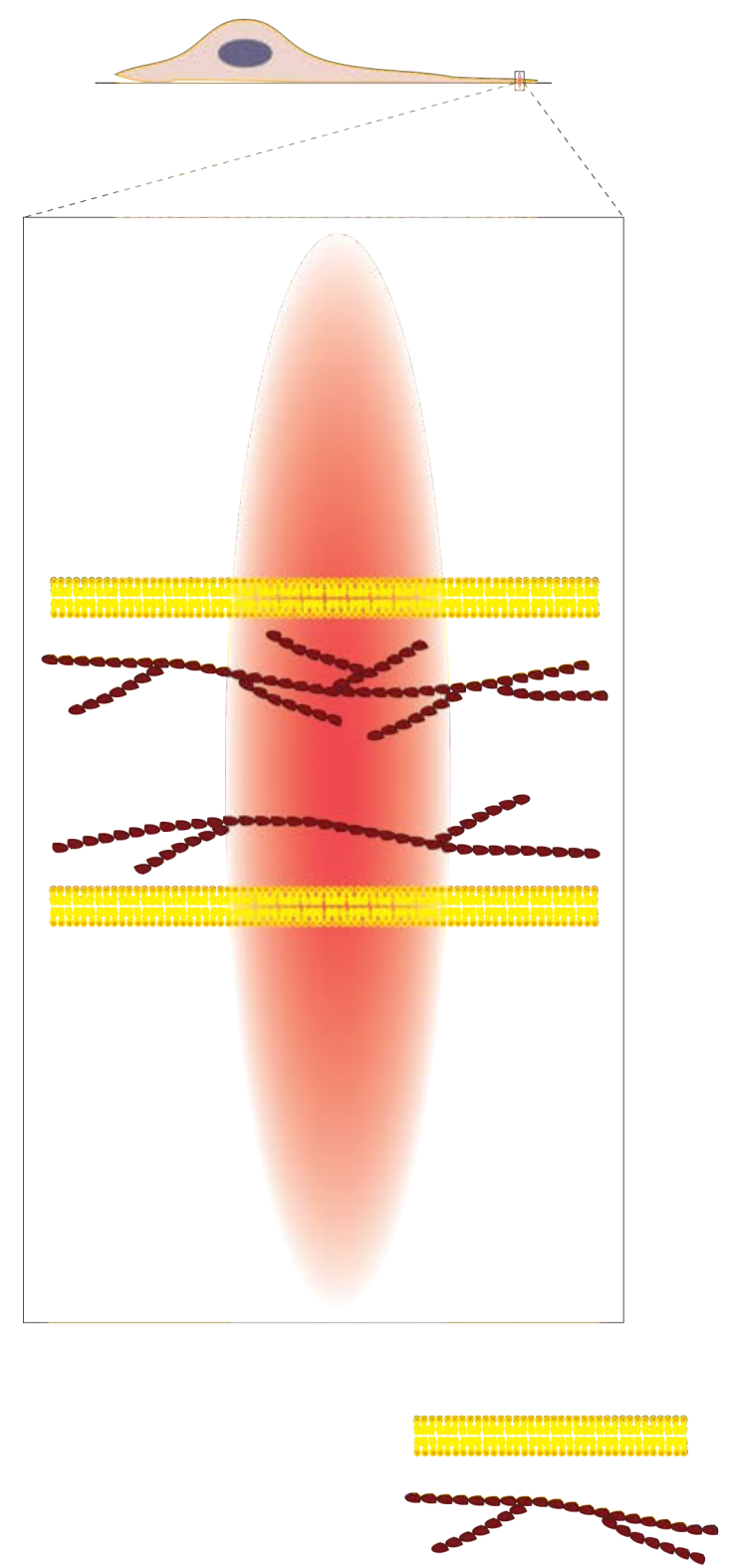

B

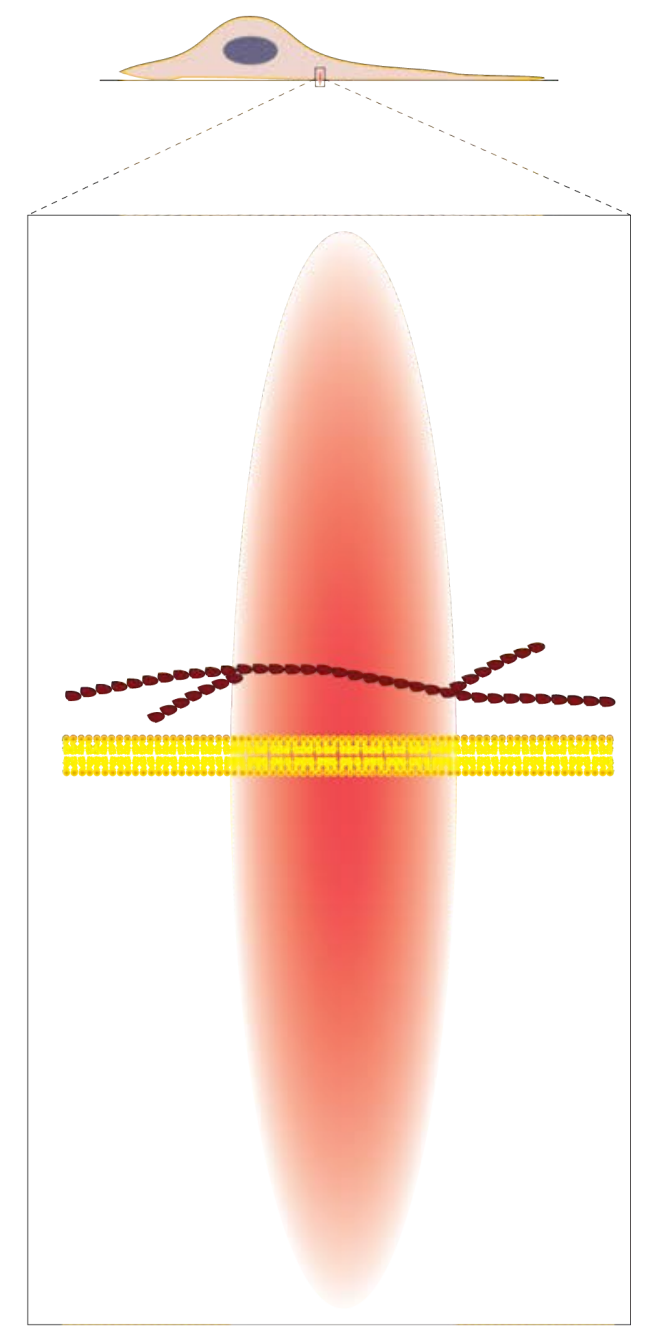

lipid bilayer

cortical actin

Figure 2.3: Schematics showing two different ways of probing lipid diffusion with STED-FCS - (A) Near the edge of a cell, the distance between apical and basal membranes is in the order of a few hundred nanometers ${ }^{56}$. In STED-FCS experiments, the focal volume is reduced only in the x-y plane (diameter varying from 240 to $40 \mathrm{~nm}$ ), rendering its $\mathrm{z}$ dimension unaltered $(\sim 640 \mathrm{~nm})$. That implies that in lamellipodia, or close to the cell edge, STED-FCS probes lipid diffusion in both membranes at the same time, averaging them. (B) Conversely, when the separation between the membranes is in the micrometer range (as it is the case in the cell body of many cells), STED-FCS ensures to probe only the basal membrane. 


\section{MATERIALS AND METHODS}

typical transient time of the labelled lipids through the confocal observation area. For each cell analysed and for each observation spot diameter d (given as the full width at half maximum (FWHM)), the calculated apparent diffusion coefficient resulted from the average of at least 5 and up to 8 repetitions of the STED-FCS measurements at the given cell. Correlation data were recorded with lipid concentrations resulting in a temporal average of particle number $\mathrm{N} \sim 0.3$ to 2 fluorescent particles for the highest $\mathrm{STED}$ power and $\mathrm{N} \sim 10$ to 30 fluorescent particles for confocal recording.

\subsubsection{STED-FCS analysis}

In FCS, the fluorescence signal, after being autocorrelated as described in Eq.(1.3), is fitted to a model which properly accounts for the physical nature of the dynamics under investigation and for the photophysics of the fluorescent label. We have used the dye Atto $647 \mathrm{~N}$ (Atto-Tec) as a label in all experiments to probe lipid diffusion. Therefore, experiments on lipid diffusion were fitted with the following model ${ }^{44}$ :

$$
G(\tau)=1+\frac{1}{N} G_{D}(\tau) G_{D S 1}(\tau) G_{D S 2}(\tau)
$$

where

$$
G_{D}(\tau)=\frac{1}{1+\left(\frac{\tau}{t_{D}}\right)^{\alpha}}
$$

describes two-dimensional, one-component diffusion dynamics and the terms

$$
G_{D S 1(2)}(\tau)=1+\frac{B_{1(2)}}{1-B_{1(2)}} \exp \left(\frac{-\tau}{t_{D S 1(2)}}\right)
$$

stand for the population of two dark states of the labelled molecule. Here, one of the dark states is the triplet state, the other being an additional dark state, characteristic of Atto647N-labelled lipids ${ }^{44}$. In the equations above, $\tau$ is the time of correlation, $N$ stands for the temporal average of the number of molecules in the focal spot, $t_{D}$ denotes the average transit time of the fluorescent molecules through the focal spot, $\alpha$ represents an anomalous diffusion exponent and $B_{1(2)}$ stand for the equilibrium fraction of molecules in the correspondent dark state, with $t_{D S 1(2)}$ being their characteristic correlation time. Diffusion was considered to be two-dimensional for that in the spatioscale relevant for STED-FCS measurements $(<240 \mathrm{~nm})$, the plasma membrane can be regarded as a two-dimensional sample.

For free diffusion, $\alpha$ equals 1 . Nevertheless, this equality does not hold for nonBrownian (anomalous) diffusion, due to the fact that for anomalous diffusion, the mean square displacement $\langle r\rangle^{2}$ of the molecule's diffusion is not linear with time $t$, i.e.

$$
\langle r\rangle^{2}=4 D_{a p p} t^{\alpha}
$$


where $D_{a p p}$ is an apparent diffusion coefficient.

Differently, for the investigation of diffusion of the Ras protein in the plasma membrane, another labelling strategy was employed. In that case, SNAP-tagged H-rasG12V proteins expressed in BHK cells were fluorescently labelled with the Silicon-containing rhodamine $(\mathrm{SiR})$ dye ${ }^{57}$. Because the binding of the dye to the SNAP-tagged protein does not occur in $100 \%$ of the cases, unbound dye may still remain on the surface of the plasma membrane. For this reason, correlation data from experiments on Ras diffusion were fitted with a model of 2-component diffusion, where one of the components undergoes free diffusion (the unbound dye) while the second component diffuses anomalously (the SNAP-tagged H-rasG12V):

$$
G(\tau)=1+\frac{1}{N} G_{D}(\tau) G_{D S}(\tau)
$$

where

$$
G_{D}(\tau)=\frac{C}{1+\left(\frac{\tau}{t_{D 1}}\right)}+\frac{(1-C)}{1+\left(\frac{\tau}{t_{D 2}}\right)^{\alpha}}
$$

describes two-dimensional, two-component diffusion dynamics and the term

$$
G_{D S}(\tau)=1+\frac{B}{1-B} \exp \left(\frac{-\tau}{t_{D S}}\right)
$$

stands for the population of the triplet state of the SiR dye. Above, $C$ is the fraction of dye molecules which are unbound, $t_{D 1}$ and $t_{D 2}$ are the average transit times of molecules through the focal spot for unbound and bound dye molecules, respectively. For the SiR dye, no population of further dark states was observed, reason why only one dark state (the triplet state) was included in the model.

Concerning experiments on lipid diffusion, all correlation data were fitted with Eqs. (2.2-2.4), where the amplitudes and characteristic times of the dark states were prefixed parameters, since they depend only on the labelled lipid and its environment, being independent of cell type or pre-treatments applied to the cells. Similarly, the amplitude and characteristic time of the triplet state were pre-fixed parameters in the analysis of Ras diffusion. Specifically, the fitted values of $t_{D}$, along with the diameter of the observation area, $d$, obtained through the calibration of the setup, allow for the calculation of the apparent diffusion coefficient $\left(D_{a p p}\right)$ :

$$
D_{a p p}=\frac{d^{2}}{8 t_{D} \ln 2}
$$

When the apparent diffusion coefficient is plotted as a function of the observation area, or analogously, of the full width at half maximum, three distinct diffusion patterns can be discerned by STED-FCS, via the so called "diffusion law": free dif- 
A
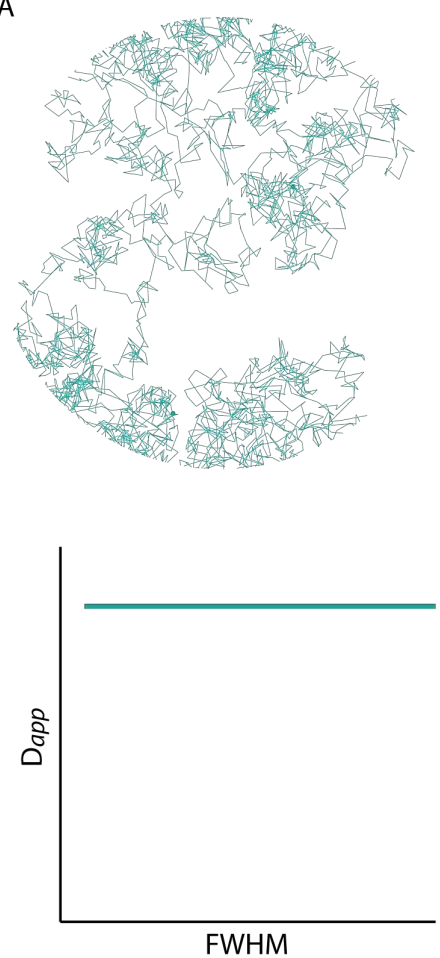

B
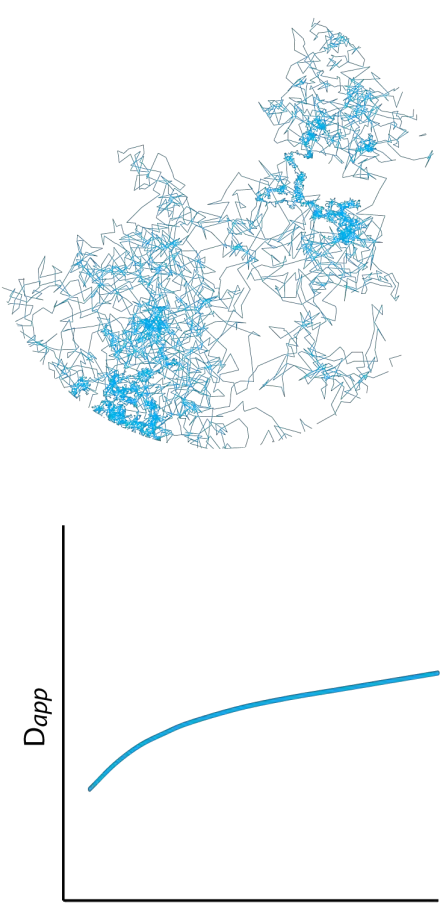

FWHM
C
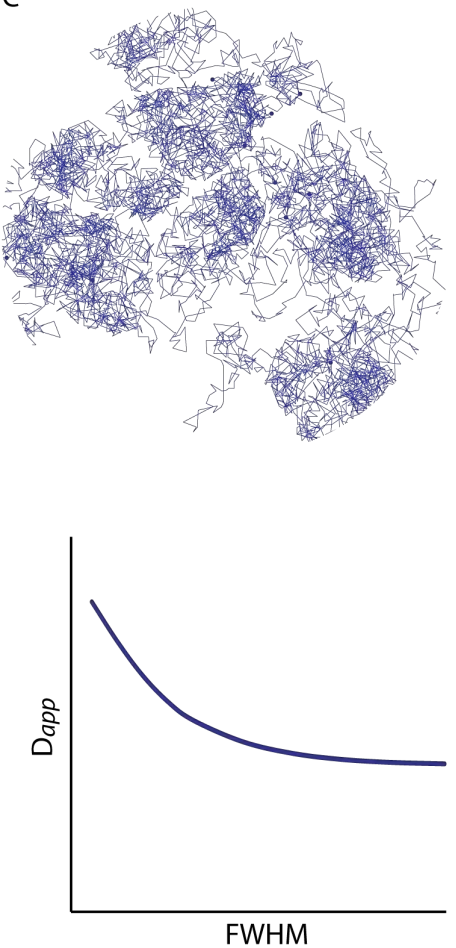

Figure 2.4: Three distinct two-dimensional diffusion patterns, discernible by STED-FCS via the diffusion law - (A) Free (Brownian) diffusion, characterized by constant $D_{a p p}$, independent of the observation diameter. (B) Trapping diffusion, marked by a decrease in $D_{a p p}$ as the FWHM is decreased (C) Compartmentalized diffusion, characterized by an increase in $D_{a p p}$ as the FWHM is decreased. 
fusion, trapping diffusion and compartmentalized (or hop) diffusion ${ }^{48,52,53}$ (Fig.2.4). Free (Brownian) diffusion is analogous to a two-dimensional random walk. Its diffusion law is characterized by constant $D_{a p p}$, that is independent of the observation diameter (FWHM). Trapping diffusion is marked by trapping events, when the diffusing molecule is transiently confined to small regions where diffusion is hindered. One of the possible physical constructs that represent this motion is a two-dimensional random walk with mobile centers of short-range, weak attraction, where the mobility of the attraction centers is much lower than the mobility of the diffusing particles. This diffusion pattern can be resolved by STED-FCS via its characteristic diffusion law, marked by a decrease in $D_{a p p}$ as the FWHM is decreased ${ }^{52}$. Compartmentalized diffusion is analogous to a two-dimensional random walk with partially reflecting barriers. In this diffusion pattern, $D_{a p p}$ increases as the FWHM is decreased, for that in this instance less barriers constrain the diffusion of molecules. Trapping and compartmentalized diffusion are two examples of anomalous diffusion.

\subsection{Sample preparation}

\subsubsection{Cell culture and transfection}

IA32, NRK, IA32 2xKD, BHK and PtK2 cells were seeded on standard glass coverslips (diameter $18 \mathrm{~mm}$, no. 1.5 thickness) to a confluence of about $60 \%$ and grown at $37^{\circ} \mathrm{C}$ in a water-saturated atmosphere of $5 \% \mathrm{CO}_{2}$ in air. Primary cultures of oligodendrocytes were prepared from postnatal day 1 mouse brains. The oligodendroglial progenitor cells were grown for 7-10 days on an astrocyte layer, followed by the shake and subsequent cultivation on the polylysine-coated glass coverslips in high-glucose Dulbecco's modified Eagle's media (DMEM), complemented with bovine serum, penicillin/streptomycin, glutamax, sodium pyruvate, triiodothyronine, L-thyroxine and B-27 supplement.

In order to transfect cultured PtK2 cells with MBP-GFP-TM construct, TransIT (Mirus Bio LLC) was used, following manufacturer instructions. Samples featuring $75 \%$ confluence were preferably taken.

BHK cells were transfected using JetPRIME transfection reagent (Polyplus - transfection) with the following plasmids: pmGFP-H-rasG12V, pmGFP-H-rasG12V-R169/ K170A or pmGFP-H-rasG12V-R128/135A. Plasmids were transfected alone or together with plasmid pcDNA3-asGal-1 in the case of depletion, or with plasmids pmRFP-Gal-1 or pcDNA3-Gal-1 in the case of overexpression.

\subsubsection{Labelling procedures}

The following labelling procedures were used in the experiments here-forth reported:

Incorporation of fluorescent lipid analogues into the plasma membrane 


\section{MATERIALS AND METHODS}

for STED-FCS measurements: To enable incorporation into cellular membranes, lipids of interest were primarily coupled to bovine serum albumin. Lipids were first reconstituted in chloroform/methanol solution, for storage in fractionated aliquots. In order to have the lipids BSA-coupled, chloroform/methanol was aspired from one aliquot, containing $75 \mathrm{nmol}$ of lipid analogues. They were then redissolved in $10 \mu \mathrm{l}$ of absolute ethanol and vortexed vigorously. $1 \mathrm{ml}$ of de-fatted BSA (in Dulbecco's Modified Eagle Medium DMEM without phenol-red, buffered with $10 \mathrm{mM}$ HEPES) was added in equimolar concentration to the lipids. Solution was centrifuged at high speed for $3 \mathrm{~min}$ to remove residual clumps. Supernatant was stored at $+8^{\circ} \mathrm{C}$. In order to incorporate BSA-coupled lipid analogues into cellular membranes, cells were first washed with 10 mM HEPES-buffered DMEM medium without phenol-red. Next, BSA-coupled lipid analogues, diluted in HDMEM, were added in appropriate concentrations onto cells. After incubation with the labelling solution for $30 \mathrm{~min}$ at $+4^{\circ} \mathrm{C}$, cells were briefly washed with HDMEM. All lipid analogues used in our experiment were labelled with the fluorescent lipophilic organic dye Atto647N (excitation max at $645 \mathrm{~nm}$, emission $\max$ at $670 \mathrm{~nm} ;$ Atto-Tec) ${ }^{44}$.

Labelling of MBP, actin and plasma membrane in oligodendrocytes and PtK2 cells: To analyse the subcellular localization of the proteins and lipids of interest in primary oligodendrocytes and PtK2 cells we performed immunocytochemistry. The following antibodies were used in this study: rabbit polyclonal anti-MBP (DakoCytomat, Carpinteria, CA, USA), phalloidin coupled to Rhodamine dye and WGA coupled to Alexa-488 (Invitrogen). Secondary antibodies were purchased from Dianova.

Labelling of F-actin in IA32, IA32 2xKD and NRK cells: Cells were seeded out on glass coverslips (no. 1.5 thickness) and allowed to adhere and spread for $20 \mathrm{~h}$ after which cells were washed 3x in PBS, and fixed in 4\% formaldehyde in PBS for 10 min. Fixed cells were subsequently washed $3 \mathrm{x}$ in PBS, and permeabilized with $0.1 \%$ Triton X-100 in PBS for 5 min. The cells were then blocked for non-specific binding by treatment in 1\% BSA in PBS for 10 min, washed 3x in PBS, and labelled with Abberior STAR 635 phalloidin (1 unit in PBS with 1\% BSA) for 10 min. Labelled cells were washed $3 \mathrm{x}$ in PBS and mounted for imaging in Mowiol mounting media (6 g glycerol,

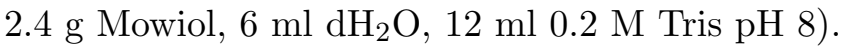

\subsubsection{Drug treatments}

The following drug treatments were performed in some of the experiments presented in chapter 3:

Cholesterol Oxidase: The cells were treated with $1 \mathrm{U} / \mathrm{ml}$ Streptomyces spec. COase (Sigma-Aldrich) in HDMEM (and washed afterwards in HDMEM) for $30 \mathrm{~min}$ under culture conditions. Treatment was performed before the insertion of the fluorescent lipid analogues into the plasma membrane. 
Blebbistatin: Treatment with $15 \mu \mathrm{M}$ Blebbistatin (EMD Millipore) in HDMEM (and washed afterwards in HDMEM) for $2 \mathrm{~h}$ under culture conditions.

Latrunculin B: Treatment with $1 \mu \mathrm{M}(100 \mathrm{nM})$ Latrunculin B (Sigma-Aldrich) in HDMEM (and washed afterwards in HDMEM) for 15 min under culture conditions was performed for IA32 cells (NRK cells). NRK cells were found to be more sensitive to this drug treatment than IA32 cells, in a way that for these cells reduction of Latrunculin B concentration to $100 \mathrm{nM}$ produced similar results to $1 \mu \mathrm{M}$. Treatment was performed before the insertion of the fluorescent lipid analogues into the plasma membrane.

CK-666: Treatment with $100 \mu \mathrm{M}$ CK-666 (EMD Millipore) in HDMEM (and washed afterwards in HDMEM) for $4 \mathrm{~h}$ under culture conditions. Treatment was performed before the insertion of the fluorescent lipid analogues into the plasma membrane. In addition, insertion of fluorescent lipid analogues and STED-FCS measurements were carried out in $100 \mu \mathrm{M}$ CK-666 in HDMEM.

\subsection{Monte Carlo simulations for STED-FCS measurements}

Via custom written routines in Matlab, we performed Monte Carlo simulations to generate fluorescence time traces of 2-dimensional diffusion of fluorescent molecules diffusing through an observation spot with a Gaussian-shaped fluorescence detection profile. The diffusion area comprised randomly sized compartments. Within a compartment the molecules were assumed to diffuse freely while crossing from one compartment to another was only possible with a given "hopping probability" $\left(\mathrm{P}_{\text {hop }}\right)$. This was implemented in the following way: If free diffusion with diffusion constant $\mathrm{D}_{\text {free }}$ would have led the lipid to cross the compartment boundary, the effective transposition would have taken place in only a fraction of such cases (being defined by $\mathrm{P}_{h o p}$ ) while in all other cases the molecule would be reflected back to the original compartment. The simulation area was a circle of $3 \mu \mathrm{m}$ diameter and the compartmentalization of this area was implemented as a Voronoi mesh on a random distribution of seed points. We defined the square root of the average compartment area as the average compartment size $(L)$. The average compartment size, the hopping probability and the free diffusion coefficient completely describe our simulation model. In most cases a simulation placed 100 independent molecules in the simulation area and took a time span of $200 \mathrm{~s}$ with $20 \mu \mathrm{s}$ time steps.

The simulated fluorescence time traces were auto-correlated and the correlation curves were fitted to the aforementioned two-dimensional diffusion model. The transient time $t_{D}$ was converted to an apparent diffusion coefficient using $D_{a p p}=d^{2} /\left(8 t_{D} \ln (2)\right)$ with diameter $d$ of the observation spot given as the FWHM. Fitting of the experimentally measured $D_{a p p}(d)$ dependence was achieved by performing simulations on iteratively finer spaced grids based on the minimization of the squared distance to the 
measured values. After reaching an approximate accuracy of $1 \times 10^{-10} \mathrm{~cm}^{2} / \mathrm{s}$ in $\mathrm{D}_{\text {free }}$, 0.01 in $\mathrm{P}_{\text {hop }}$ and $5 \mathrm{~nm}$ in $L$, the program stopped running the iterations and displayed the most pertinent set of fitted parameters achieved. 


\section{3}

\section{Lipid compartmentalization at the plasma membrane}

In the last three decades, many important discoveries have changed our view of the plasma membrane structure ${ }^{15,22,30,33,44,58}$. In this context, the cytoskeleton has emerged as a key modulator of the lateral diffusion of membrane proteins. The analogous cytoskeleton-dependent lipid diffusion has been proposed ${ }^{26}$, but this remains a highly debatable concept ${ }^{29}$. However, here we report the observation of cytoskeleton-modulated compartmentalized diffusion of phospholipids in the plasma membrane of living cells attained using a fluorescent lipid analogue in combination with STED-FCS. Compartmentalized diffusion was in particular found to be dependent on the F-actin branching nucleator Arp2/3. These findings provide solid evidence for that the Arp2/3-branched actin cytoskeleton plays a pivotal role in the dynamical architecture of the plasma membrane, potentially regulating its fundamental processes.

\subsection{Lipid compartmentalization hypothesis}

The fluid mosaic model for biological membranes proposed by Singer and Nicolson in $1972^{6}$ is a milestone in membrane research. Nevertheless, novel methods for probing dynamics of molecular structures developed in the last decades have brought a wealth of biological insight that contradicts the Singer-Nicholson model, particularly in the case of the plasma membrane ${ }^{58}$. For example, Brownian diffusion of proteins and lipids, being one of the main assumptions of that model, has been shown to be largely inaccurate $^{15,22,30,33,44,47,58}$. A variety of experimental techniques have shown that the lateral motion of proteins in the plasma membrane is constrained by different mechanisms, many of them being ultimately connected to the cortical actin cytoskeleton ${ }^{15,30,52,59}$. In addition, SPT experiments utilizing gold particles or quantum dots (QDs) have further suggested that even phospholipid diffusion in the outer leaflet of the plasma 


\section{LIPID COMPARTMENTALIZATION AT THE PLASMA MEMBRANE}

membrane is constrained ${ }^{27,60}$, presumably by the cortical actin cytoskeleton.

In view of these findings, which suggest the structure and organization of the plasma membrane to be strongly coupled to the cortical actin cytoskeleton, the picket-fence model was proposed ${ }^{26}$. This model hypothesizes that the actin filaments underlying the plasma membrane act as barriers that constrain the diffusion of transmembrane proteins. The agglomeration of such proteins, anchored to actin filaments, would in turn constrain the diffusion of other proteins and of lipids, creating thus cytoskeletonbased compartments throughout the plasma membrane (Fig.3.1). The notion that the cytoskeleton plays a main role in diffusion through plasma membrane and in plasma membrane function finds resonance in important findings in membrane research. Studies performed on model membranes bound to a layer of actin mesh showed that diffusion of lipids and proteins was hindered by the mesh, and that such reduction in mobility was correlated to the actin density ${ }^{61}$. Nanoclusters of numerous membrane-associated proteins were reported to be colocalized with actin ${ }^{62}$ and assembled via interaction with dynamic actin filaments at the cortex ${ }^{33}$. Finally, the cytoskeleton was found to be directly responsible for controlling the activity of structures like mechanosensitive ion channels ${ }^{63}$.

Nevertheless, the picket-fence model has encountered several obstacles for its full acceptance $^{29,31,64}$. While compartmentalized diffusion of membrane proteins in living cells was observed by different methods, the same has not been confirmed for phospholipids, which was thus far only supported by SPT experiments ${ }^{27,60}$. In these experiments, gold particles ${ }^{27}$ and QDs $^{60}$ were so far employed in order to reach the required temporal resolution. Such probes are artefact prone due to their prominent size (orders of magnitude bigger than the lipids themselves), and due to the difficulty in validation of their valence towards the target molecule, inducing possible oligomerization ${ }^{31,64}$. In addition, the validity of SPT reports on compartmentalized diffusion was drawn into question by a study showing that the irregularity of plasma membrane topography can induce apparent compartmentalized diffusion, as observed by $\mathrm{SPT}^{65}$.

\subsection{Detecting lipid compartmentalized diffusion via STED- FCS}

In order to elucidate the dilemma regarding lipid compartmentalized diffusion (also called hop diffusion), we have applied STED-FCS to probe the diffusion of a phospholipid analogue labelled with a small organic dye (Fig.3.3) in living cells, at high temporal and spatial resolution. STED-FCS allows a systematic probing of molecular diffusion with spatial resolution ranging from diffraction-limited $240 \mathrm{~nm}$ to below 40 $\mathrm{nm}$, a range that is comparable in size to the postulated actin cytoskeleton mediated compartments $^{28,54}$. 

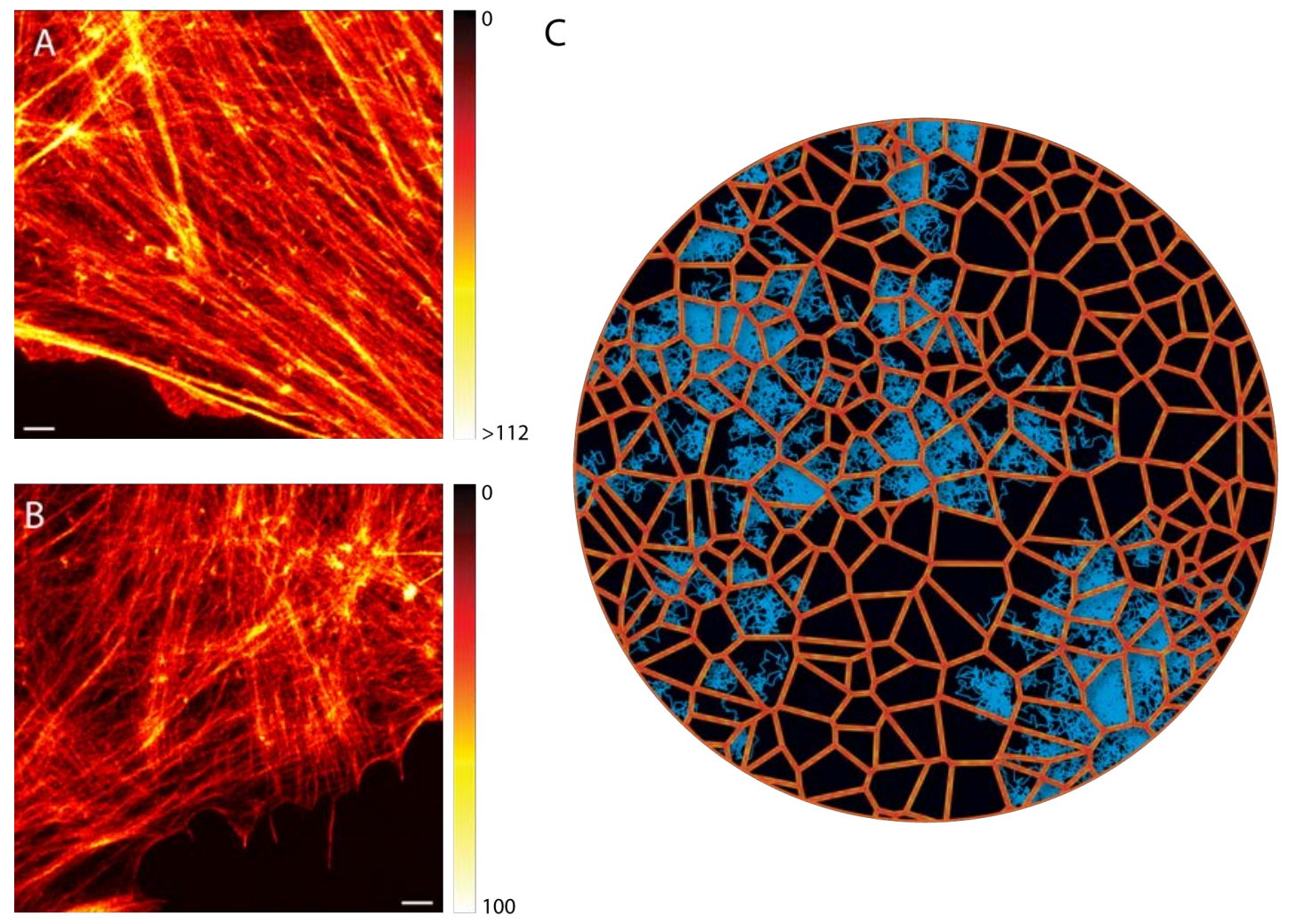
00

Figure 3.1: Plasma membrane compartmentalization hypothesis - (A, B) Representative STED images of F-actin labelled with Abberior STAR 635 phalloidin in NRK and IA32 cells, respectively. Scale bar is $1 \mu \mathrm{m}$. (C) Schematics showing branched networks that partially confine two-dimensional diffusion of molecules. Within compartments molecules are assumed to diffuse freely, and in the event of hitting the boundaries of a compartment, transposition to the adjacent compartment occurs with a certain hopping probability. 


\section{LIPID COMPARTMENTALIZATION AT THE PLASMA MEMBRANE}
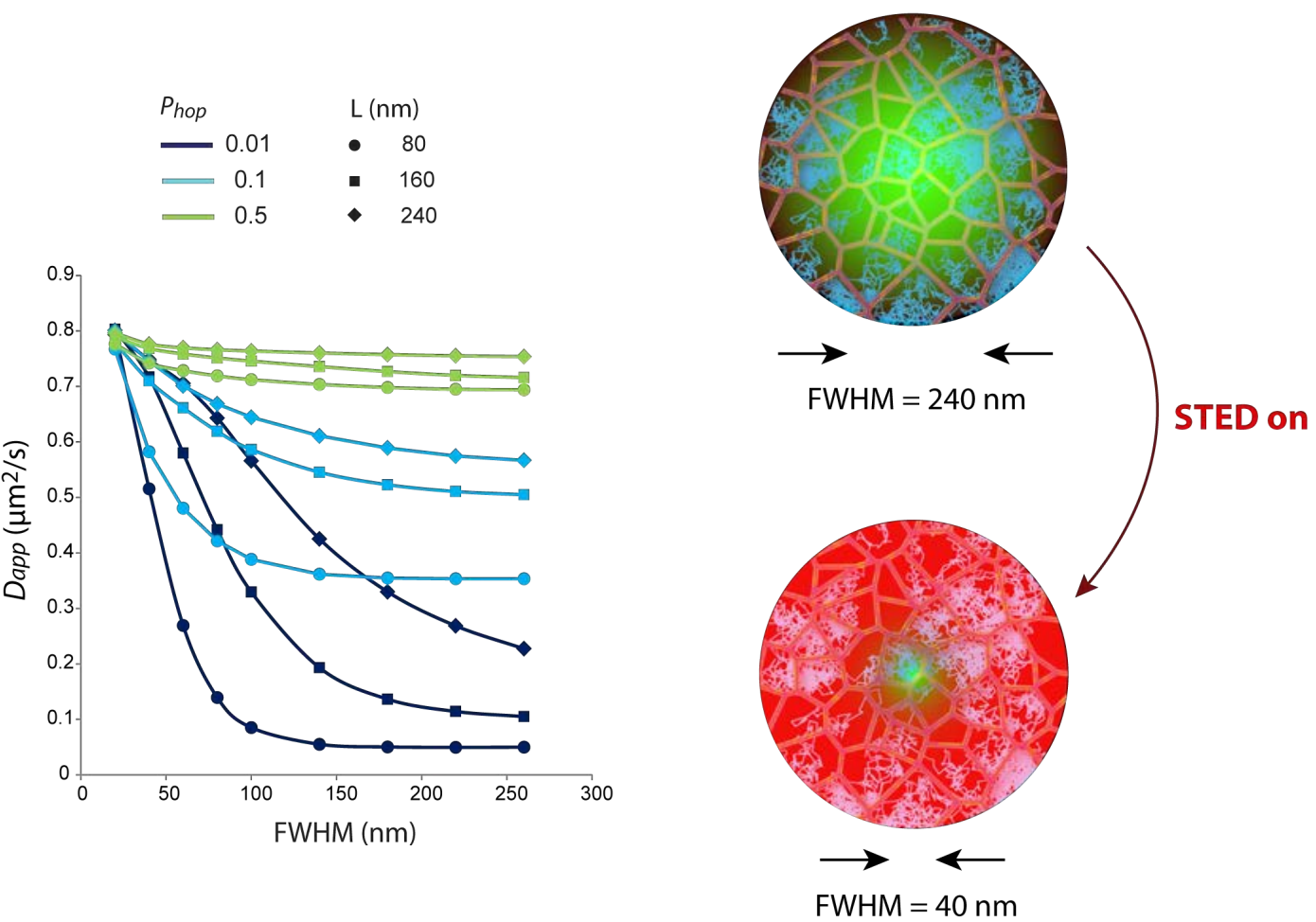

Figure 3.2: In-silico STED-FCS experiments - Simulations show characteristic dependencies of the apparent diffusion coefficient $D_{a p p}$ on the full width at half maximum (FWHM) of the observation spot, assuming a model for compartmentalized diffusion as depicted in Fig.3.1 C. As the FWHM is decreased, the apparent diffusion coefficient increases. Characteristic compartment size $(L)$, free diffusion coefficient $\left(\mathrm{D}_{\text {free }}\right)$ and hopping probability $\left(\mathrm{P}_{\text {hop }}\right)$ define the diffusion model. As it can be observed, only strong confinement (small $\mathrm{P}_{\text {hop }}$ ) renders clear patterns of compartmentalized diffusion. 


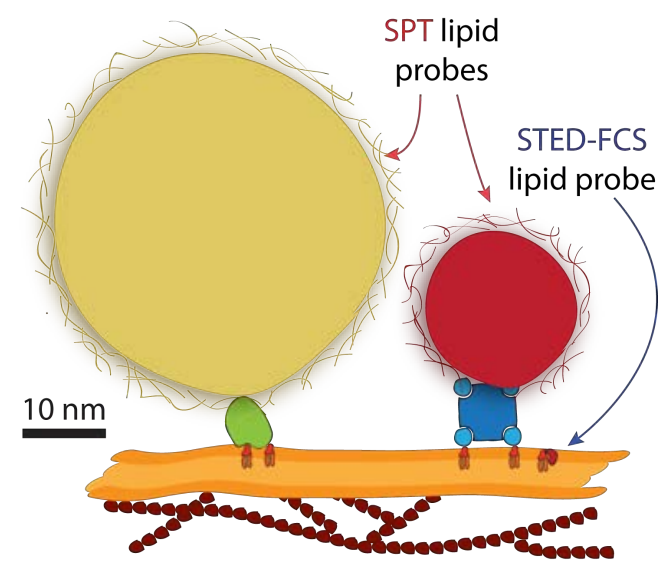

Figure 3.3: Lipid probes used in SPT and STED-FCS experiments - Schematics of lipid probes used in SPT and in STED-FCS, put in perspective. Gold particle ( $\sim 40$ $\mathrm{nm}$ in diameter) linked to lipid by Fab antibody and QD ( $\sim 20 \mathrm{~nm}$ in diameter) linked to lipid via streptavidin, as often used in SPT. Both probes are functionalized via polymer coating, which further enhances their effective size. Possible oligomerization induced by SPT probes is illustrated for the QD. STED-FCS lipid probe stands for a fluorescent lipid analogue ( $\sim 1 \mathrm{~nm}$ in diameter). Sketch with courtesy of Mathias Clausen, (University of Oxford)

Validating the method, we first performed in-silico STED-FCS experiments of diffusion within a heterogeneously compartmentalized lattice with a characteristic average compartment length $L$. Within compartments molecules were assumed to diffuse freely with a diffusion coefficient $\mathrm{D}_{\text {free }}$, while transposing compartment boundaries was possible with a certain "hopping probability" $\mathrm{P}_{\text {hop }}$. These experiments show that the compartment barriers slow down diffusion for observation diameters much bigger than the compartments themselves. The reason for this is the increasing number of barriers the probe has to transpose while diffusing through the focal spot. As the observation diameter is decreased, the apparent diffusion coefficient significantly increases because in this instance free diffusion inside the compartment is probed (Fig.3.2). Specifically for small $\mathrm{P}_{h o p}$, i.e. strong confinement, compartmentalized diffusion can be clearly distinguished from free diffusion, which is characterized by a constant diffusion coefficient that is independent of the FWHM.

While compartmentalized diffusion of fluorescent phospholipid analogues was not observed in initial STED-FCS studies in live PtK2 cells ${ }^{44,47}$, we now report for the first time that it becomes evident via STED-FCS in two live cell types, NRK cells and in IA32 cells (Ink4a/Arf (-/-) mouse fibroblasts) ${ }^{66}$ (Fig.3.4). In this study, we used the phospholipid di-palmitoyl-phosphoethanolamine (DPPE) labelled with Atto647 $\mathrm{N}^{44,47}$. Prominent compartmentalized diffusion of lipids has previously been reported by SPT studies $^{27,60}$ in these cell types, but those results had never been validated by any other 


\section{LIPID COMPARTMENTALIZATION AT THE PLASMA MEMBRANE}

technique or by the use of a less invasive probe, remaining thus far debatable.
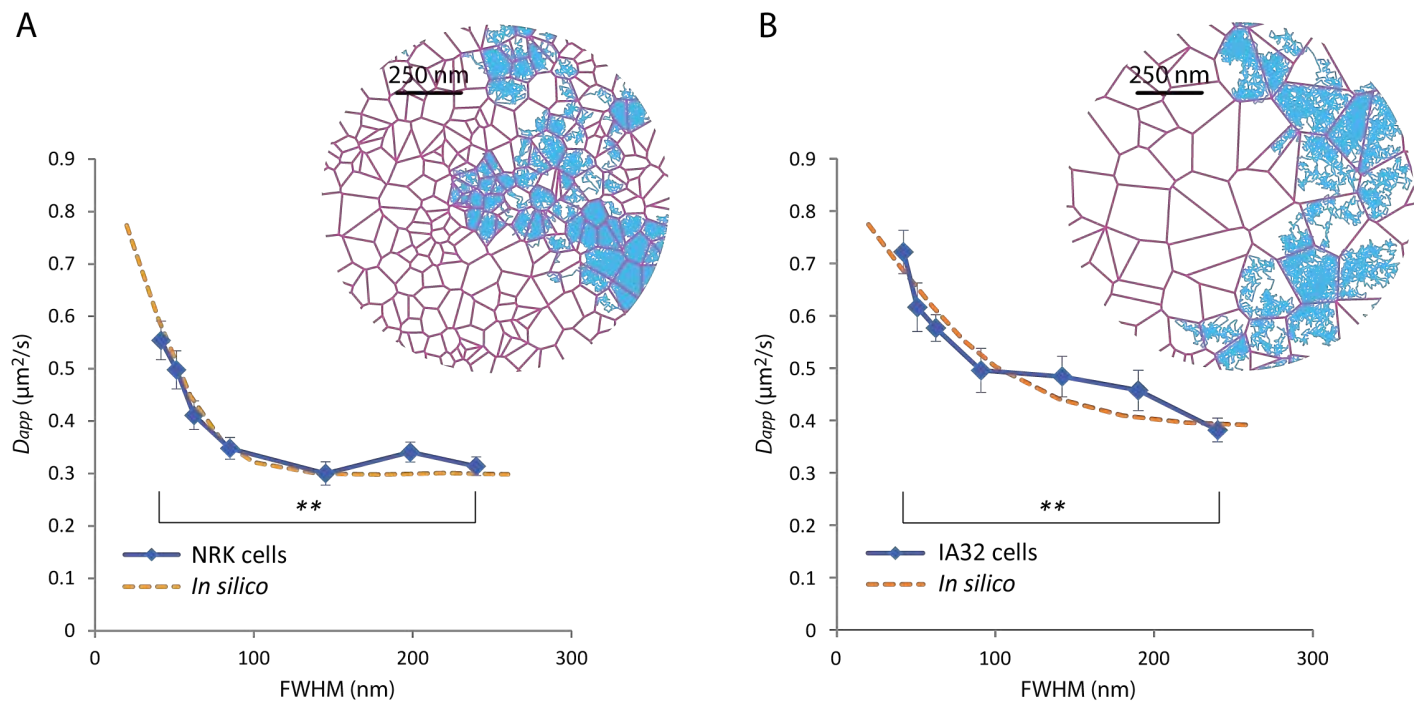

Figure 3.4: Experimental observation of lipid compartmentalized diffusion by STED-FCS - (A, B) $D_{a p p}(\mathrm{FWHM})$ dependencies for DPPE-Atto647N diffusion in NRK and IA32 cells, respectively. Clear compartmentalized diffusion patterns are observed. ${ }^{\star \star} P$ $<0.01$ (unpaired $t$ test). Error bars are standard error of the mean. In $\mathrm{A}, n=32$ cells; in $\mathrm{b}, n=33$ cells. Fitting of the experimental data resulted for both cell types $\mathrm{D}_{\text {free }}=$ $0.8 \mu \mathrm{m}^{2} / \mathrm{s}$ and $\mathrm{P}_{h o p}=0.1$. The characteristic compartment size was found to be $80 \mathrm{~nm}$ for NRK and $150 \mathrm{~nm}$ for IA32 cells. Insets: representative voronoi lattices (red) relative to the correspondent compartment sizes as well as simulated diffusion trajectories (blue) correspondent to the fitted parameters. Scale bars: $250 \mathrm{~nm}$.

These STED-FCS experiments resulted in a clear pattern of compartmentalized diffusion as indicated by a significant increase of $D_{a p p}$ towards smaller observation diameters. Monte Carlo simulations were used to fit the experimental data to a model of free diffusion constrained by a heterogeneously compartmentalized lattice. The fitting parameters that statistically best described the data were for both cell types $\mathrm{D}_{\text {free }}=$ $0.8 \mu \mathrm{m}^{2} / \mathrm{s}$ and $\mathrm{P}_{h o p}=0.1$, and $L=80 \mathrm{~nm}$ for NRK cells and $L=150 \mathrm{~nm}$ for IA32 cells.

\subsection{Investigation of molecular mechanisms underlying lipid compartmentalization}

In order to assess the underlying causes for compartmentalized diffusion, we have systematically performed STED-FCS experiments on IA32 and NRK cells where the actin cytoskeleton was modulated, membrane cholesterol was depleted, or myosin II activity 
was inhibited. Actin cytoskeleton modulation was achieved by treating cells with either Latrunculin B (latB) or CK-666. These drugs perturb the actin cytoskeleton through different mechanisms: while latB prevents the polymerization of all types of F-actin networks by sequestering G-actin, CK-666 is a recently discovered ${ }^{67}$ small molecule that interferes with the dynamic cortical F-actin networks by specific inhibition of the Arp2/3 complex, the actin nucleator that enables the creation of branched actin networks ${ }^{68}$.

\subsubsection{Cytoskeleton modulation significantly impacts lipid compart- mentalized diffusion}

Cells treated with latB showed faster DPPE diffusion, markedly NRK cells (Fig.3.5 A, C). Compartmentalized diffusion was still observed, although not strictly statistically significant at confidence level 95\% (Fig 3.5 B, E, and Appendix). Contrastingly, cells treated with CK-666 showed remarkably faster DPPE diffusion, where the diffusion coefficient assumed approximately a constant value, independent of the observation diameter (Fig.3.5 A, B, C, E), which is indicative of free diffusion.

In order to further investigate the effects of Arp2/3 depletion in compartmentalized lipid diffusion, we performed STED-FCS experiments on IA32 MEFs that had been additionally depleted of p34Arc and Arp2 by use of a lentiviral shRNA knockdown system (IA32 2xKD cells) ${ }^{66}$. The resulting dependence of $D_{a p p}(\mathrm{FWHM})$ is compatible with free diffusion (Fig.3.5 D), reinforcing that Arp2/3 depletion suppresses compartmentalized diffusion. We have observed that DPPE diffusion in 2xKD is slower than in CK-666-treated IA32 cells (Fig.3.5 E), possibly due to reduced membrane tension and increased curvature in $2 \mathrm{xKD}$ cells, although this remains to be proven. In fact, these two different Arp2/3 depletion approaches render distinct conformations of the F-actin cytoskeleton and overall cell shape (Fig.3.6).

In order to assess possible differences between lipid diffusion in the apical and basal membrane of cells, due to the distinct conformations of the apical and basal sub-membranous actin cytoskeleton ${ }^{56}$, we performed STED-FCS measurements in the basal membrane under the cell body of NRK cells. These measurements resulted in the observation of faster and slightly less compartmentalized diffusion, as compared to the measurements encompassing both membranes near the cell edge (Fig.3.7). This is consistent with a lower cortical actin density at the basal membrane, as compared to the apical membrane, and/or to a lower cortical actin density in the cell body, as compared to the edge of the cell ${ }^{56}$. 


\section{LIPID COMPARTMENTALIZATION AT THE PLASMA MEMBRANE}
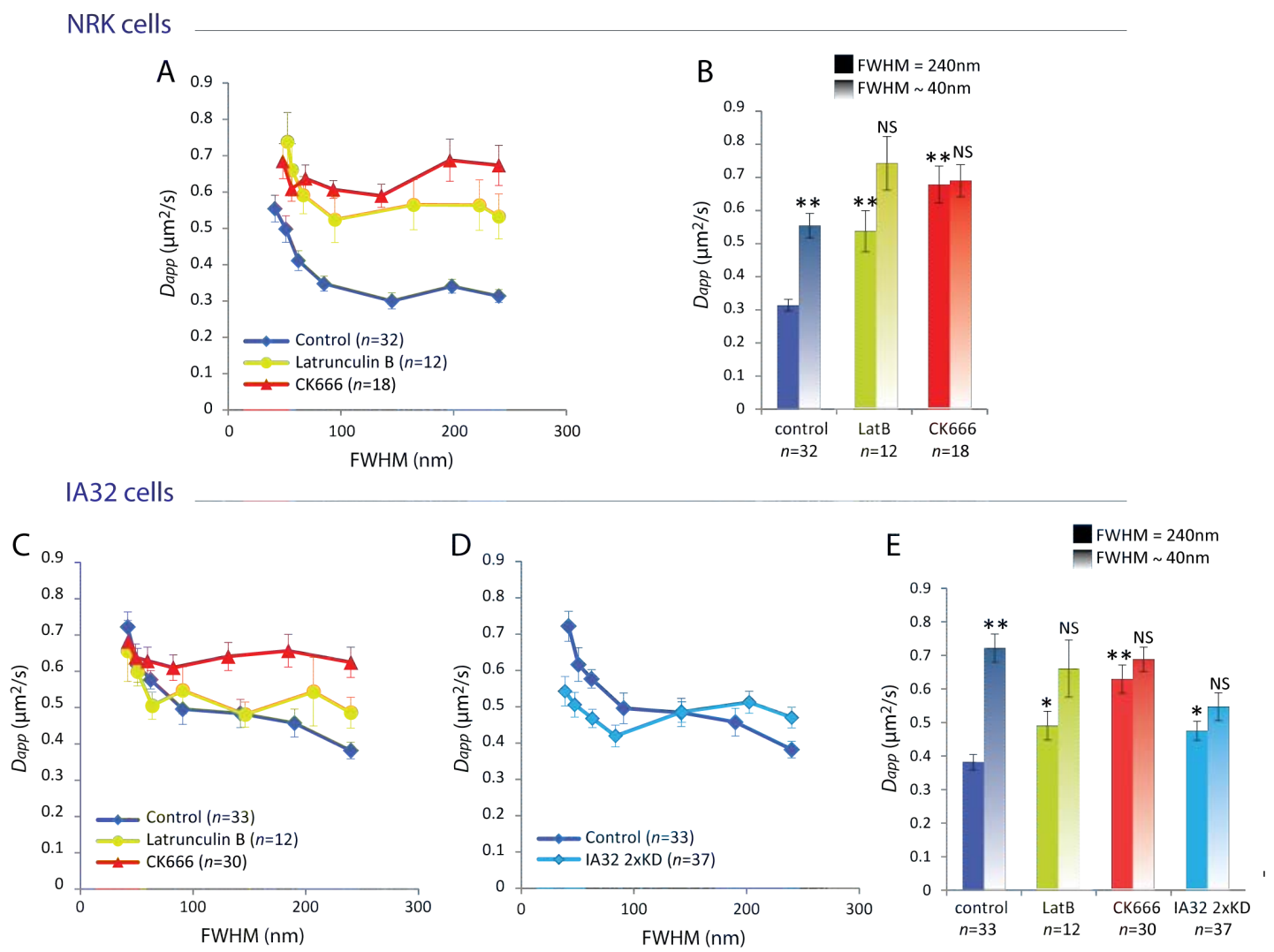

Figure 3.5: Cytoskeleton depletion effect on lipid diffusion in NRK and IA32 cells - (A) Effect on lipid diffusion of Latrunculin B and CK-666 treatment in NRK cells. (B) Summary of the data in A. (C) Effect on lipid diffusion of Latrunculin B and CK666 treatment in IA32 cells. (D) Comparison of DPPE diffusion patterns, as observed by STED-FCS, between IA32 and IA32 2xKD cells. (E) Summary of the data in C and D. ${ }^{\star \star} P$ $<0.01,{ }^{\star} P<0.05$, NS, not significant (two-tailed unpaired $t$ test). Error bars are standard error of the mean. Results of the statistical test on top of the columns representing FWHM $\sim 40 \mathrm{~nm}$ refer to comparison with the precedent column $(\mathrm{FWHM}=240 \mathrm{~nm})$. Results of the statistical test on top of the columns representing FWHM $=240 \mathrm{~nm}$ refer to comparison with the control $(\mathrm{FWHM}=240 \mathrm{~nm})$. $n$ stands for the number of cells. 


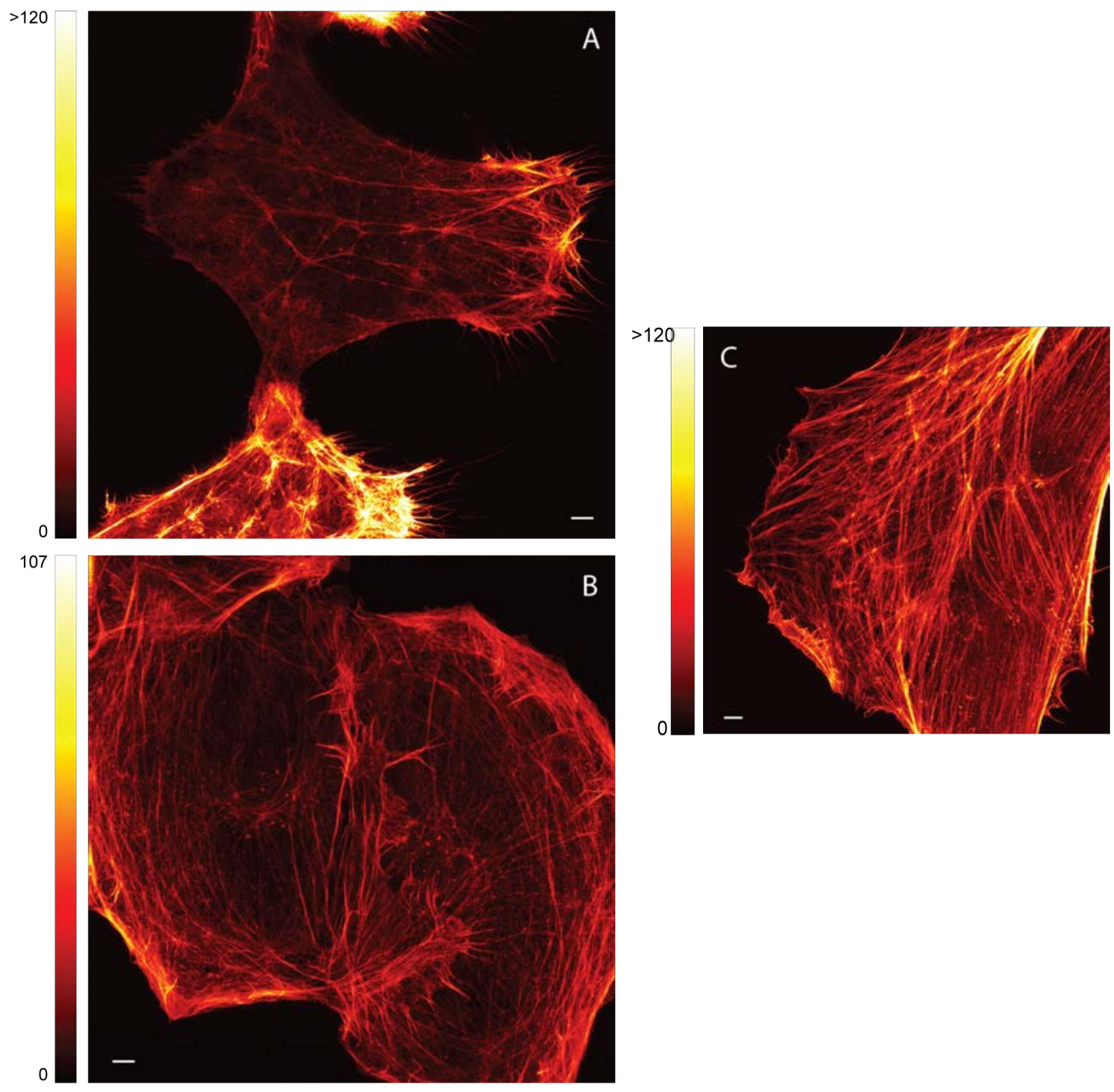

Figure 3.6: Arp 2/3 depleted cells - (A, B) Representative STED images of F-actin labelled with Abberior STAR 635 phalloidin in CK-666-treated IA32 cells and 2xKD cells, respectively. (C) Untreated IA32 cell, for comparison. Scale bars are $3 \mu \mathrm{m}$. 


\section{LIPID COMPARTMENTALIZATION AT THE PLASMA MEMBRANE}
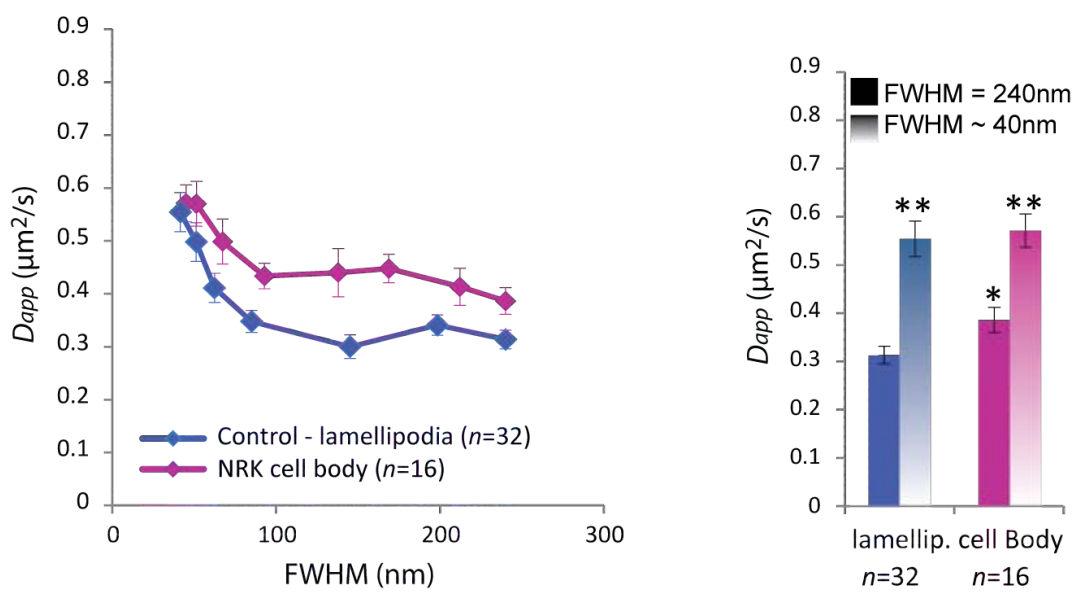

Figure 3.7: Different patterns of compartmentalized diffusion in NRK cells Diffusion in lamellipodia and in the cell body, as observed by STED-FCS. ${ }^{\star \star} P<0.01,{ }^{\star} P$ $<0.05$ (two-tailed unpaired $t$ test). Error bars are standard error of the mean. Results of the statistical test on top of the columns representing FWHM $\sim 40 \mathrm{~nm}$ refer to comparison with the precedent column $(\mathrm{FWHM}=240 \mathrm{~nm})$. Results of the statistical test on top of the columns representing FWHM $=240 \mathrm{~nm}$ refer to comparison with the control (FWHM $=240 \mathrm{~nm}) . n$ stands for the number of cells.

\subsubsection{Cholesterol depletion and myosin II inhibition do not affect lipid compartmentalized diffusion}

Inhibition of myosin II with blebbistatin and cholesterol depletion using cholesterol oxidase (COase) had no effect on DPPE diffusion, in neither NRK nor IA32 cells (Fig.3.8). Myosin II works as a regulator for actin dynamics, allowing the cell to tune its cortical actin mesh disposition. Blebbistatin is a small molecule which specifically inhibits myosin II. The result that diffusion was unaffected by blebbistatin treatment rules out myosin-based contractility as a reason for the observed compartmentalized diffusion. In addition, the observation that DPPE compartmentalized diffusion was unaltered after cholesterol depletion with COase indicates that compartmentalization of lipids is not due to cholesterol-mediated interactions. It has been reported that cholesterol depletion may lead to detachment of the plasma membrane from the actin cytoskeleton as a secondary effect ${ }^{5963}$. However, the level of cholesterol depletion used in our experiments was not high enough in order to make this side effect significant.

\subsection{Discussion}

The fact that cytoskeleton-modulated, compartmentalized lipid diffusion could be clearly observed by STED-FCS in NRK and in IA32 cells, but not in previously investigated PtK2 cells ${ }^{44,47}$, poses the question of whether the membrane compartmentalization 
NRK cells
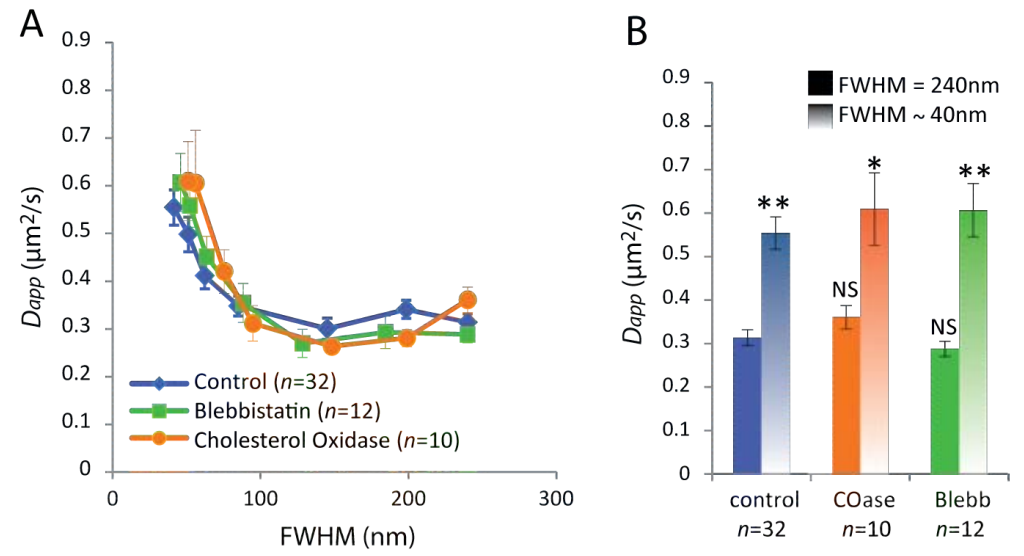

IA32 cells
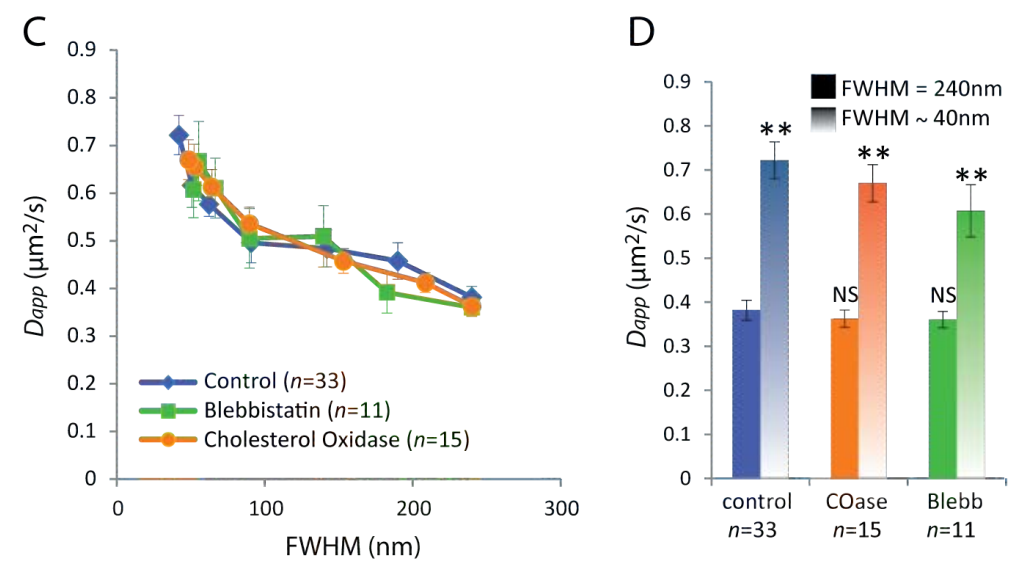

Figure 3.8: Cholesterol depletion and myosin II inhibition do not alter lipid compartmentalization - (A, C) Cholesterol depletion and myosin II inhibition in NRK and IA32 cells, respectively. No significant change in diffusion pattern occurred after treatment with Cholesterol Oxidase and Blebbistatin. (C, D) Summary of the data. ${ }^{\star \star} P$ $<0.01,{ }^{\star} P<0.05$, NS, not significant (two-tailed unpaired $t$ test). Error bars are standard error of the mean. Results of the statistical test on top of the columns representing FWHM $\sim 40 \mathrm{~nm}$ refer to comparison with the precedent column $(\mathrm{FWHM}=240 \mathrm{~nm})$. Results of the statistical test on top of the columns representing FWHM $=240 \mathrm{~nm}$ refer to comparison with the control $(\mathrm{FWHM}=240 \mathrm{~nm})$. Here, $n$ stands for the number of cells. 


\section{LIPID COMPARTMENTALIZATION AT THE PLASMA MEMBRANE}

exerted by the cytoskeleton is inherently cell type-specific, or a generic phenomenon. To answer this question, we notice that compartmentalized and free diffusion is only distinguishable by STED-FCS in cases where the average compartment size falls within the size range assessed by this technique, and further that the confinement strength is sufficiently large. In addition, compartmentalized diffusion combined with trapped diffusion might further fade the observation of that diffusion mode ${ }^{53}$.

A combination of these factors can explain the observation of apparent free diffusion for PtK2 cells, as our simulation model successfully describes DPPE diffusion on PtK2 cells ${ }^{47}$ with $L=25 \mathrm{~nm}, \mathrm{P}_{\text {hop }}=0.25$ and $\mathrm{D}_{\text {free }}=0.7 \mu \mathrm{m}^{2} / \mathrm{s}$ (Fig.3.9). This explanation is further experimentally supported by the fact that CK-666-treated PtK2 cells show significantly faster diffusion of DPPE, as compared to untreated cells (average diffusion coefficient of $0.63 \mu \mathrm{m}^{2} / \mathrm{s}$, contrasting to $0.40 \mu \mathrm{m}^{2} / \mathrm{s}$ in untreated PtK2 cells), indicating that also in this cell type lipid diffusion is modulated by the Arp2/3-branched actin networks.

A

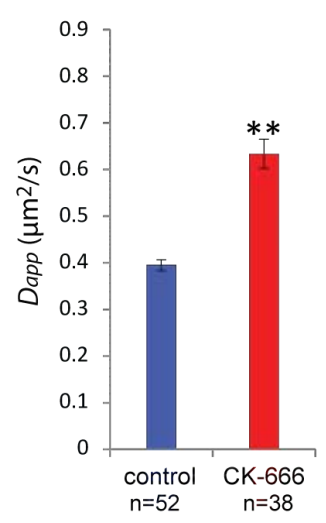

B

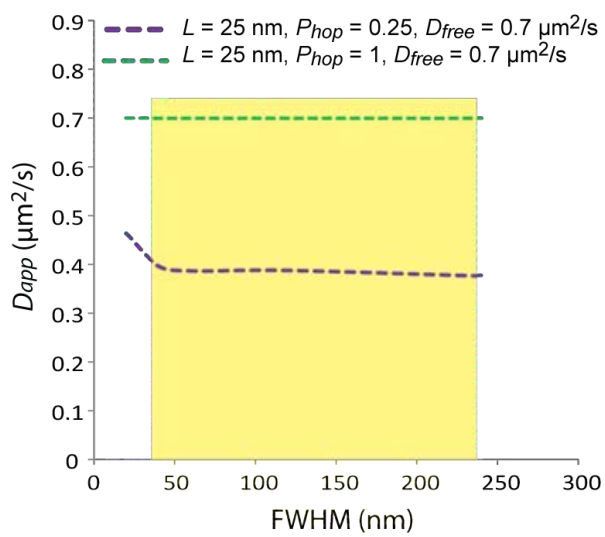

Figure 3.9: Simulation model for phospholipid diffusion in PtK2 cells - (A) Experimental DPPE diffusion coefficient for diffusion in the plasma membrane of untreated and CK-666-treated PtK2 cells (FWHM $=240 \mathrm{~nm})$. ${ }^{\star \star} P<0.01$ (two-tailed unpaired $t$ test). (B) In silico STED-FCS experiments for an average compartment size $L=25 \mathrm{~nm}$, free diffusion coefficient $\mathrm{D}_{\text {free }}=0.7 \mu \mathrm{m}^{2} / \mathrm{s}$ and hopping probability $\mathrm{P}_{\text {hop }}=0.25$ and $=1$. The in silico data describes accurately the experimental FCS data for untreated and CK-666treated PtK2 cells. The curve correspondent to $\mathrm{P}_{h o p}=1$ can also be obtained by setting $L \gg 240 \mathrm{~nm}$. The region in yellow corresponds to the currently accessible FWHM range for STED-FCS experiments. As can be observed, very small compartment sizes preclude the observation of compartmentalized diffusion.

Previous studies by SPT using gold particles ${ }^{27}$ have shown that phospholipids (DOPE) are compartmentalized in the plasma membrane of NRK cells in compartments with a mean diameter of $230 \mathrm{~nm}$, within which they diffuse freely with a median diffusion coefficient of $5.4 \mu \mathrm{m}^{2} / \mathrm{s}$. Also, previous studies by SPT using QDs ${ }^{60}$ have 
shown that most of DPPE molecules are confined in IA32 cells within compartments with a mean diameter of $150 \mathrm{~nm}$, within which they diffuse freely with a median diffusion coefficient of $0.6 \mu \mathrm{m}^{2} / \mathrm{s}$. Now, for the first time, those studies are validated with a different technique, utilizing a small organic dye as a lipid label. Differences in the compartment sizes and diffusion coefficient within compartments, as determined by different techniques, may stem from the different probes utilized in each case. Nevertheless, our data is not satisfactorily described by our simulation model if the diffusion coefficient within compartments is fixed to $5.4 \mu \mathrm{m}^{2} / \mathrm{s}$ (Fig.3.10), as reported by Kusumi and colleagues ${ }^{27}$. This suggests that the observation of such fast diffusion might be induced by the large gold particle utilized in that experiment, which wobbles in the extra-cellular medium where the viscosity is lower than in the plasma membrane by two orders of magnitude ${ }^{31}$.

A

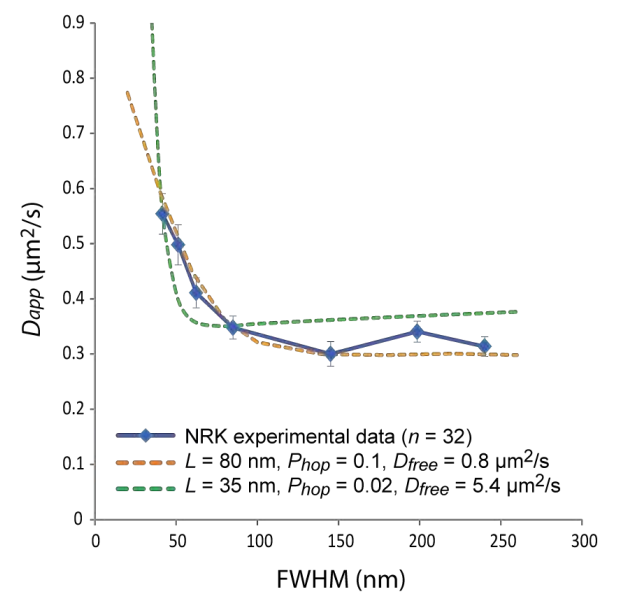

B

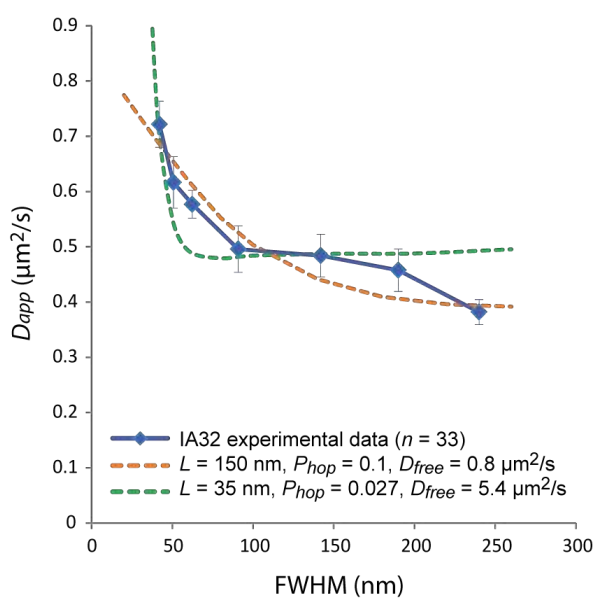

Figure 3.10: Comparing simulation parameters for phospholipid diffusion in cells - (A, B) Experimental DPPE diffusion coefficient for diffusion in the plasma membrane of NRK and IA32 cells, respectively, and in-silico experiments based on diffusion through a heterogeneously compartmentalized lattice. In both cases, the blue solid line corresponds to the experimental data, the orange dashed line corresponds to the curve that best fitted our experimental data (without pre-defined constraints), and the green dashed line corresponds to the best fit obtained with the constraint $\mathrm{D}_{\text {free }}=5.4 \mu \mathrm{m}^{2} / \mathrm{s}$. In both plots, $n$ stands for the number of measured cells.

In summary, STED-FCS has allowed a minimally invasive observation of lipid compartmentalized diffusion on the plasma membrane of living cells and has revealed with unprecedented detail the underlying mechanisms that regulate such compartmentalization. In particular, this study conclusively pinpoints the Arp2/3-complex as a regulator of the compartmentalization observed in the plasma membrane. In fact, Arp $2 / 3$ is a major component in the orchestration of the numerous tasks performed by the actin cytoskeleton, being involved in polymerization, organization and recycling of the 


\section{LIPID COMPARTMENTALIZATION AT THE PLASMA MEMBRANE}

actin networks ${ }^{69}$. Furthermore, recent studies using the Arp2/3 inhibitor CK-666 and Arp2/3-depleted mammalian cells have revealed new roles of the Arp2/3-dependent cortical cytoskeleton in a variety of cellular processes including matrix sensing, cytoplasmic streaming, spindle positioning and cell-cell junction regulation ${ }^{66,70-72}$. Here, we demonstrate for the first time the influence of Arp2/3 regulation on lipid diffusion in living cells.

Following these results, we are compelled to infer that compartmentalization of lipids by the actin cytoskeleton is a fundamental cellular process, independent of cell type. Partial compartmentalization of membrane lipids and proteins may be associated, for example, with localized signalling ${ }^{28}$. In this context, yet another form of confinement promoted by the cytoskeleton, i.e., one-dimensional diffusion of integral membrane proteins along actin filaments, was shown to enhance the interaction probability of less abundant proteins, thereby potentially triggering important cellular events ${ }^{73}$. The determination of the precise structural and molecular mechanisms by which the cytoskeleton performs lipid compartmentalization, as well as the mechanisms by which it may be implied in fundamental processes such as cell signalling, are exciting open questions that emerge from this evolving picture of the plasma membrane.

Concluding, a model for membrane organization that does not acknowledge the symbiosis between the plasma membrane and the cytoskeleton will be biologically oversimplified. Supporting the picket-fence model, our findings extend the organizing principles proposed by the fluid mosaic model by incorporating the underlying mechanism that governs the "fluidity" of the mosaic. 


\section{Transient trapping of plasma membrane molecules}

A remarkable feature of plasma membrane dynamics is that the diffusion of many molecules is marked by trapping events ${ }^{44,47,74-76}$, which are characterized by transient confinement of molecules in small regions of the plasma membrane. This diffusion pattern is discernible from free and compartmentalized diffusion by STED-FCS via the assessment of the apparent diffusion coefficient, which for trapping diffusion decreases as the observation diameter is decreased ${ }^{52,53}$ (Fig.2.4 B). The precise nature of these trapping events, i.e., the broad principles that govern this type of anomalous diffusion, are poorly understood. Trapping diffusion has been attributed to, for example, transient and cholesterol-assisted binding to other less mobile molecules or molecular complexes (often referred to as nanodomains), interactions of membrane molecules with the cortical cytoskeleton and long-range attraction between membrane proteins ${ }^{44,47,77,78}$. Furthermore, transient confinement of membrane molecules happens on spatio-temporal scales that challenge currently available biophysical methods ${ }^{22,25}$. Theoretical works can recreate trapping events based on a construct of nano-sized regions of higher viscosity ${ }^{52,53}$, centers of short-range, weak attraction distributed across the plane of diffusion ${ }^{79}$, as well as centers of repulsion ${ }^{80}$. This variety of possibilities illustrate the diversity of physico-chemical backgrounds on which trapping diffusion may rely. In this chapter, we examine two biological systems where trapping events (or their absence) play a crucial role in plasma membrane function. Next, the theoretical principles underlying the characteristic diffusion law for trapping diffusion are briefly discussed. 


\subsection{Lipid diffusion in myelin sheath}

The myelin sheath is a modified extension of the plasma membrane of specific cells, which insulates the axons of neurons by enwrapping them in a multilayered fashion. While Schwann cells originate myelin sheath in the peripheral nervous system, oligodendrocytes are responsible for providing the central nervous system with this special membrane. Each myelin-generating cell envelops only one segment of the axon. Between adjacent myelin-wrapped parts of the axon, short portions are left uncovered, the so-called nodes of Ranvier ${ }^{81}$. The combination of myelinated segments of axon interspersed among nodes of Ranvier enables saltatory conduction, allowing high-speed nerve impulse propagation ${ }^{82}$.

\subsubsection{Myelin membrane sheets: a unique biological membrane}

The history of myelin research is interconnected with the history of plasma membrane investigation. The unit membrane model, proposed by J.D. Robertson, was inspired by electron micrographs featuring a remarkable continuity of the laminar myelin sheath, forming a spiral around cross-sectional images of axons ${ }^{5}$. When Singer and Nicolson proposed the fluid mosaic structure of biological membranes, they considered myelin as a possible exception of their model, for which the fluid character of membranes would not apply ${ }^{6}$.

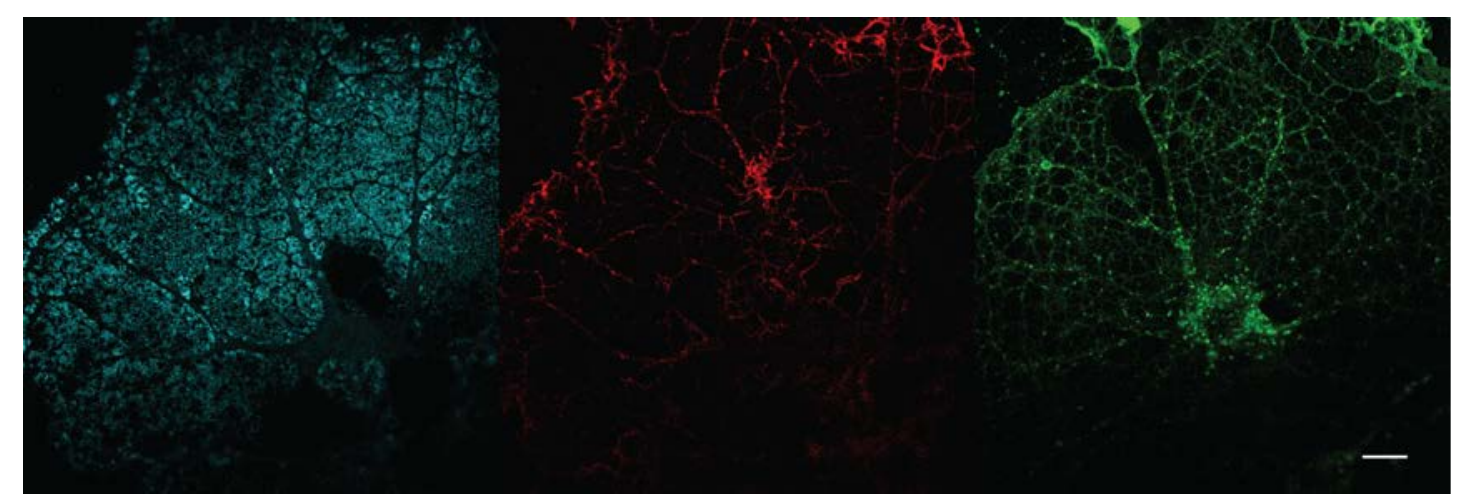

Figure 4.1: Oligodendrocytes - Cultured oligodendrocytes fixed with PFA 4\% + GTA $0.25 \%$ on day 5 and immunostained for MBP (cyan), rhodamine-phalloidin (red) and WGA488 leptin (green), for imaging of compact myelin sheets, actin and glycoproteins, respectively. Glycoproteins and actin are absent in oligodendrocyte compact sheets. Scale bar is $10 \mu \mathrm{m}$. Images with courtesy of Olena Steshenko (Max Planck Institute for Experimental Medicine, Göttingen).

Many factors contribute to the distinction of myelin membrane sheets from ordinary plasma membranes: high abundance of lipids ( $80 \%$ of its dry weight) and low amount of proteins, as well as a peculiar lipid composition (enrichment in long-chain 
fatty acids, cholesterol and galactosylceramides ${ }^{83}$ ) are characteristics of myelin membrane. Importantly, myelin membranes are not supported by the sub-membranous actin meshwork, as are plasma membranes in general. Instead, a meshwork comprising myelin basic protein (MBP) underlies this membrane, defining the morphology of its architecture ${ }^{84,85}$. In this context, myelin emerges as a differential system in the investigation of membrane-organizing mechanisms.

Here, we observe for the first time lipid dynamics in myelin membrane, as well as address the influence of the underlying MBP meshwork on lipid mobility.
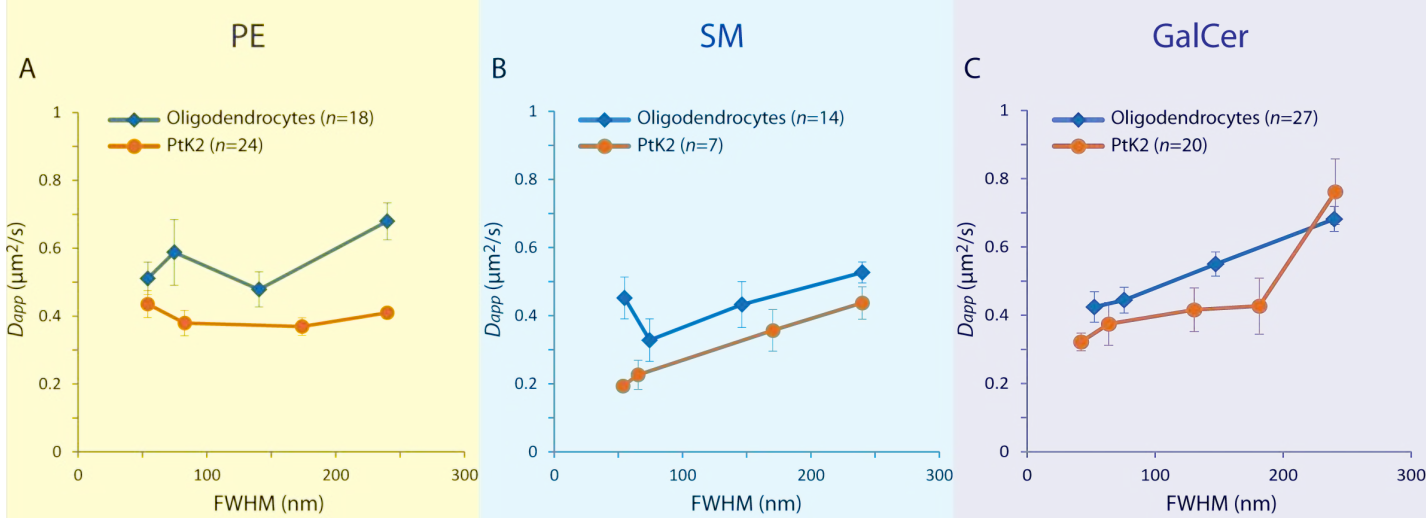

D

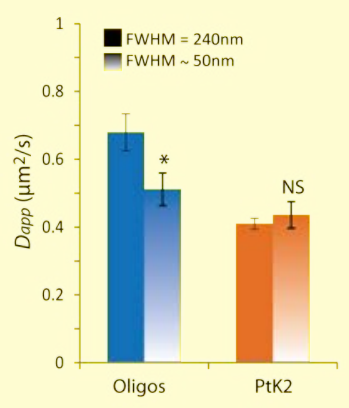

$E$

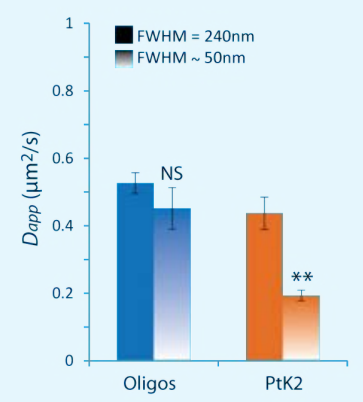

$\mathrm{F}$

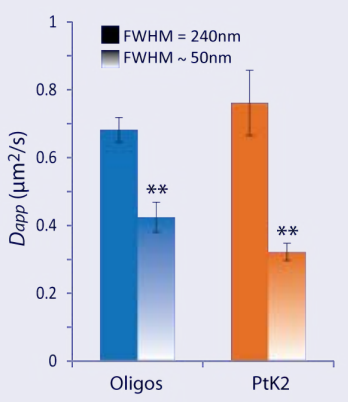

Figure 4.2: Lipid diffusion in oligodendrocyte membrane sheets from oligodendrocytes and in PtK2 cells - (A-C) $D_{a p p}(\mathrm{FWHM})$ dependencies for respectively PE, SM and GalCer diffusion in oligodendrocytes and PtK2 cells $(n$ stands for the number of cells). (D-F) Summary of the data. ${ }^{\star \star} P<0.01,{ }^{\star} P<0.05$, NS, not significant (two-tailed unpaired $t$ test). Error bars are standard error of the mean. Results of the statistical test on top of the columns representing FWHM $\sim 50 \mathrm{~nm}$ refer to comparison with the precedent column $(\mathrm{FWHM}=240 \mathrm{~nm})$.

In order to assess lipid dynamics in myelin, we investigated lipid diffusion in primary cultures of oligodendrocytes, which form prominent myelin membrane sheets (Fig.4.1). Upon culturing on the glass cover slips these cells reproduce two major domains of in vivo myelin: flat compact sheets that recapitulate myelin sheath, and non-compact 


\section{TRANSIENT TRAPPING OF PLASMA MEMBRANE MOLECULES}

membrane processes. We used fluorescent analogues of the saturated sphingolipids sphingomyelin (SM) and galactosylceramide (GalCer), for that the correspondent endogenous lipids are abundant in myelin and therefore may be assumed to play an important role in myelin function. For comparison with a different lipid group, we as well utilized a fluorescent analogue of the saturated phosphoglycerolipid phosphatidylethanolamine (DPPE, here denoted by PE). The diffusion of these lipids has been previously investigated by STED-FCS in the epithelial PtK2 cell line, where free diffusion of PE and prominent trapping diffusion of $\mathrm{SM}^{44}$ and GalCer ${ }^{47}$ was observed.

Lipid diffusion was observed to be in general faster in myelin membranes of oligodendrocytes than in PtK2 cells (Fig.4.2). The phosphoglycerolipid PE was observed to freely diffuse in both oligodendrocytes and PtK2 cells, with a small variation of the measured apparent diffusion coefficients for different observation diameters (Fig.4.2 A,D). However, contrasting to lipid diffusion observed in PtK2 cells, sphingolipids did not undergo strong trapping diffusion in oligodendrocyte membranes. In fact, SM diffusion was observed to be much less hindered in this system (Fig.4.2 B,E). GalCer featured trapping diffusion in myelin, however slightly less prominent than in PtK2 cells. A possible measure for the assessment of the significance of trapping events in diffusion is the comparison between apparent diffusion coefficients measured at different observation diameters. In this context, GalCer trapping in oligodendrocytes is shown to be weaker than in PtK2 cells for that the difference in $D_{a p p}$ at FWHM=240 nm and at FWHM $50 \mathrm{~nm}$ is greater for PtK2 cells. These results suggest less sources of confinement affecting lipid diffusion in compact membranes sheets of oligodendrocytes than in the epithelial PtK2 cell.

\subsubsection{Myelin basic protein modulates lipid diffusion in myelin mem- brane sheets}

In the previous chapter it was demonstrated how the sub-membranous actin cortex effectively compartmentalizes the plasma membrane, modulating lipid diffusion. In this context, a system comprising a plasma membrane which is not bound to the actin cortex, as myelin membrane sheets, may be expected to feature special characteristics. In compact myelin membrane sheets, the underlying MBP meshwork possibly allows membrane-organizing mechanisms distinct from the organizing principles of plasma membranes supported by the actin cortex. In order to selectively investigate the influence of the underlying MBP meshwork on lipid diffusion, we observed lipid diffusion in PtK2 cells transfected with a chimeric construct consisting of MBP fused to GFP-label and transmembrane domain (MBP-TM-GFP) ${ }^{84}$. In the transfected cells, MBP gets anchored within the endoplasmatic reticulum membrane, and upon interactions with the negatively charged lipids in the opposed plasmalemma, it creates MBP-positive $(\mathrm{MBP}+)$ islands, highlighted by GFP. As in oligodednrocytes, MBP+ islands are de- 
prived of cortical actin and glycoproteins ${ }^{84}$ (Fig.4.3). The portions of plasma membrane of PtK2 cells within these platforms are henceforth referred to as "PtK2 MBP+".
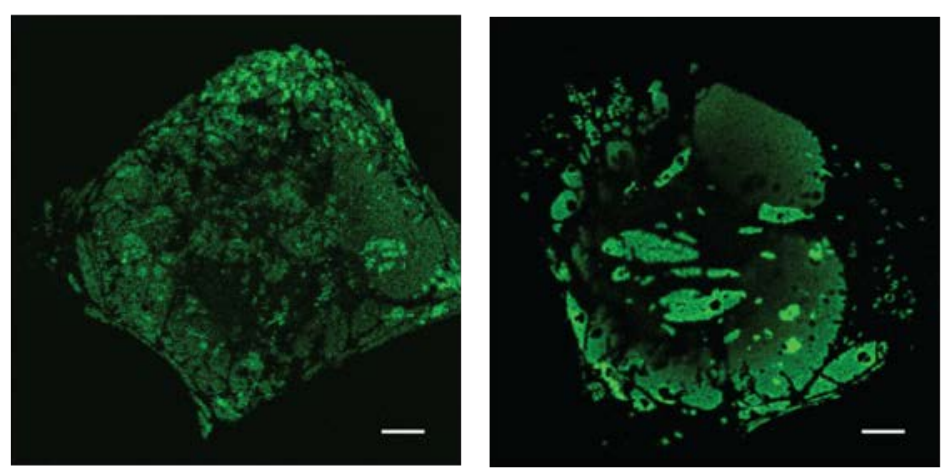

Figure 4.3: PtK2 cells transfected with the chimeric construct MBP-TM-GFP - Visualization of platforms of MBP meshwork between the inner leaflet of the plasma membrane and the endoplasmatic reticulum in PtK2 cells. Scale bars are $10 \mu \mathrm{m}$. Images with courtesy of Olena Steshenko (Max Planck Institute for Experimental Medicine, Göttingen).

For each lipid type under investigation, diffusion in PtK2 MBP + regions was significantly faster than diffusion in both oligodendrocytes and control PtK2 cells (Fig.4.4). In addition, PE, SM and GalCer were observed to undergo free diffusion in PtK2 MBP+ regions, where trapping was completely eliminated. This result strongly indicates that the insertion of MBP meshwork platforms onto the inner leaflet of the plasma membrane decreases the sources of membrane lateral heterogeneities, promoting free diffusion.

\subsubsection{Discussion}

The fact that lipid diffusion is unconfined and remarkably faster in PtK2 MBP + regions, as compared to control PtK2 cells, is in line with less confined and faster lipid diffusion in oligodendrocytes, as compared to PtK2 cells. Moreover it indicates that lipid diffusion is modulated by the underlying MBP meshwork. In myelin membrane sheets of oligodendrocytes, fast diffusion may be hindered by the abundance of long-chain fatty acids, which might increase membrane viscosity. The role of MBP in promoting fast, unconfined diffusion is not assumed here to be necessarily an active one. Possibly, the simple extrusion of the actin cytoskeleton facilitates unconfined diffusion. In fact, the actin cytoskeleton, besides promoting compartmentalization in the plasma membrane, has also been observed to be involved in trapping events. For example, SM trapping diffusion in PtK2 cells was shown to be cytoskeleton-assisted, since treatment of cells with Latrunculin B suppressed trapping ${ }^{47}$. Nonetheless, trapping of lipids with large sugar head-groups such as gangliosides (or GalCer) were found to be less dependent on 

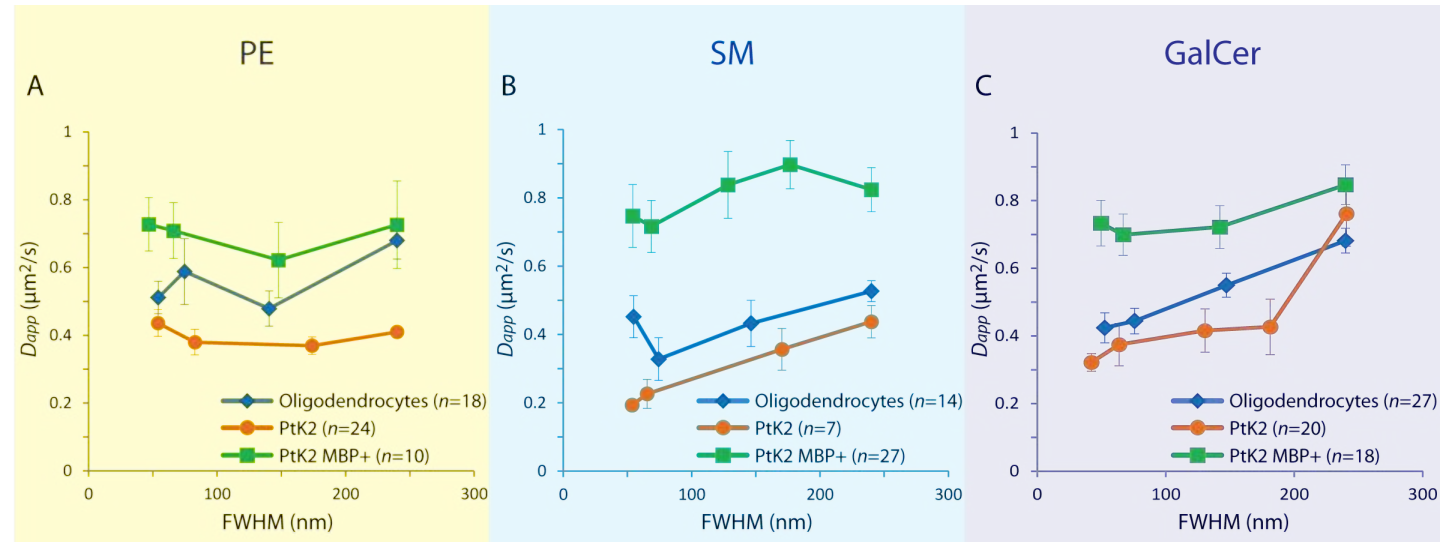

D

E

$\mathrm{F}$
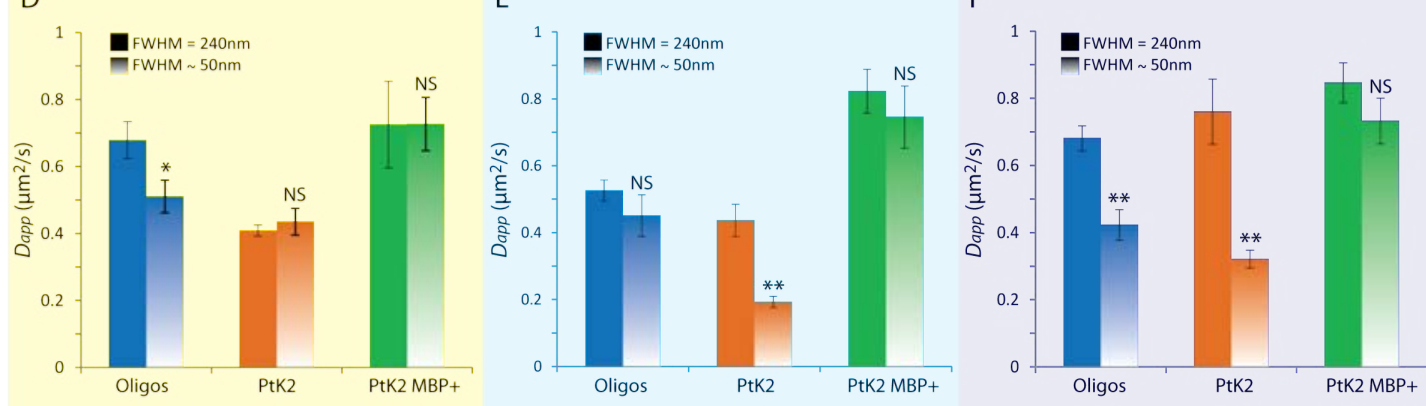

Figure 4.4: Lipid diffusion modulated by MBP - (A-C) $D_{a p p}(\mathrm{FWHM})$ dependencies for respectively PE, SM and GalCer diffusion in oligodendrocytes, PtK2, and PtK2 MBP+ cells ( $n$ stands for the number of cells). (D-F) Summary of the data. ${ }^{\star \star} P<0.01,{ }^{\star} P<0.05$, NS, not significant (two-tailed unpaired $t$ test). Error bars are standard error of the mean. Results of the statistical test on top of the columns representing FWHM $\sim 50 \mathrm{~nm}$ refer to comparison with the precedent column $(\mathrm{FWHM}=240 \mathrm{~nm})$. 
the cytoskeleton ${ }^{47}$.

Interestingly, in PtK2 $\mathrm{MBP}+$ regions, all lipids investigated here diffuse (freely) with approximately the same diffusion coefficient. Thus, in these regions, distinctions in diffusion characteristics that were before inherent to lipid types are not present any more, with different lipids diffusing similarly. Yet in this context, it is worth noting that in PtK2 MBP+ regions, lipids diffuse at a diffusion coefficient of approximately $0.8 \mu \mathrm{m}^{2} / \mathrm{s}$. This value corresponds also to the fitted free diffusion coefficient $\left(\mathrm{D}_{\text {free }}\right)$ for PE diffusion within compartments, in NRK and IA32 cells (Fig. 3.4). Furthermore, as demonstrated in the previous chapter, upon CK-666 treatment PE diffuses freely in NRK and IA32 cells with an apparent diffusion coefficient of approximately $0.65 \mu \mathrm{m}^{2} / \mathrm{s}$. All that provides compelling indications that the underlying cortical cytoskeleton plays a critical role in membrane lateral heterogeneities, since either its depletion or extrusion result in free diffusion at similar diffusion coefficients.

However, these results also suggest the existence of other sources of confinement in the plasma membrane, additionally to the actin cytoskeleton, since also in myelin membrane sheets of oligodendrocytes GalCer trapping diffusion was observed, although slightly less prominent than in PtK2 cells. Since this lipid is abundant in myelin, it may be speculated that the interaction of GalCer with other lipids, and possibly with proteins, plays a special role in the insulating and protective functions ascribed to myelin. The diffusion in myelin membrane sheets of lipids with long-chain fatty acids (21-24C) in their structure is an important question to be addressed in future research, due to their high abundance in this membrane. Here, we have employed fluorescent lipid analogues with short-chain fatty acids (16-18C) in their structures, since the incorporation of long-chain lipid analogues into the plasma membrane of cells is technically challenging. New protocols and methods facilitating this incorporation may further help to shed light on membrane dynamics in this important biological system.

\subsection{Nanodomain assembly of Ras proteins}

Ras is a family of proteins related to many signal transduction pathways. The malfunction or abnormalities in these pathways may compromise numerous activities necessary for cell survival and replication, possibly allowing the abnormal cell proliferation that leads to cancer. Due to that fact, many signalling proteins were first discovered by the identification of their encoding genes, whose mutation was associated with cancer $^{86}$. Ras were among the first oncogenes to be discovered, and in fact about $20 \%$ of all tumours present mutations in one of the Ras genes ${ }^{87}$. In humans, three Ras genes encode four distinct proteins: H-Ras, N-Ras, K-Ras4A and K-Ras4B, where KRas4 $\mathrm{A}$ and K-Ras4B are originated from alternative splicing ${ }^{88}$. In its activated state, 


\section{TRANSIENT TRAPPING OF PLASMA MEMBRANE MOLECULES}

Ras binds to guanosine triphosphate, whereas in its neutral state it binds to guanosine diphosphate. These two distinct states are in addition marked by conformational changes. Oncogenic mutations make Ras proteins to be in a perpetual activated state and consequently impair cell replication ${ }^{86}$.

Although interaction mechanisms of Ras proteins have been studied in detail ${ }^{89}$, relatively little is known about specific interactions of these proteins in their native membranous environment. In the first steps of signal transduction, Ras is attached to the inner leaflet of the plasma membrane by a 15 -carbon farnesyl lipid tail ${ }^{90}$. Membranebound Ras forms transient nanoclusters ${ }^{91}$, whose stability was reported to be regulated by nanocluster-scaffolding proteins such as galectin- ${ }^{92}$. Importantly, nanoclustering of Ras proteins was identified as a necessary step in Ras-mediated signal transduction ${ }^{93}$.

Mutants that represent different conformers of Ras on the membrane were observed to have different affinities to the nanocluster-scaffolding protein galectin- ${ }^{94}$. In order to make a direct assessment of whether Ras conformers feature different signalling characteristics, we investigated the impact of galectin-1 regulation on dynamics of three H-Ras conformers in living cells. In this study, we used H-RasG12V and the orientation-mutants (mutants that feature a different conformation) H-RasG12V R169A/K170A and H-RasG12V R128A/R135A. Previous studies indicated that the mutant H-RasG12V R128A/R135A was less reactive to galectin-1, while the mutant H-RasG12V R169A/K170A was more reactive ${ }^{94}$.

For endogenous levels of galectin-1, STED-FCS revealed trapping diffusion for all three conformers, consistent with anomalous diffusion experienced by these proteins upon transient clustering (Fig. 4.5). Upon down-regulation of galectin-1, trapping was suppressed in the diffusion of all three conformers. In particular, the orientationmutant H-RasG12V R169A/K170A featured a completely different diffusion pattern upon galectin-1 depletion, resembling compartmentalized diffusion. This is consistent with galectin-1 regulation affecting more significantly the dynamics of this orientationmutant than the other conformers. Upon up-regulation of galectin-1, trapping diffusion was observed in all conformers, slightly more prominently for the mutant H-RasG12V R169A/K170A.

In summary, STED-FCS evidenced that the diffusion of Ras proteins is regulated by galectin-1, consistent with previous studies which suggested this protein to stabilize Ras nanoclusters. Diffusion of the orientation-mutant H-RasG12V R169A/K170A was observed to be more affected by the cellular concentration of galectin- 1 than the diffusion of the other conformers. Since Ras-mediated signal transduction relies on nanocluster assemblies, these results indicate that mutations that affect only the conformation of Ras proteins may significantly impact their ability to mediate signalling events. 


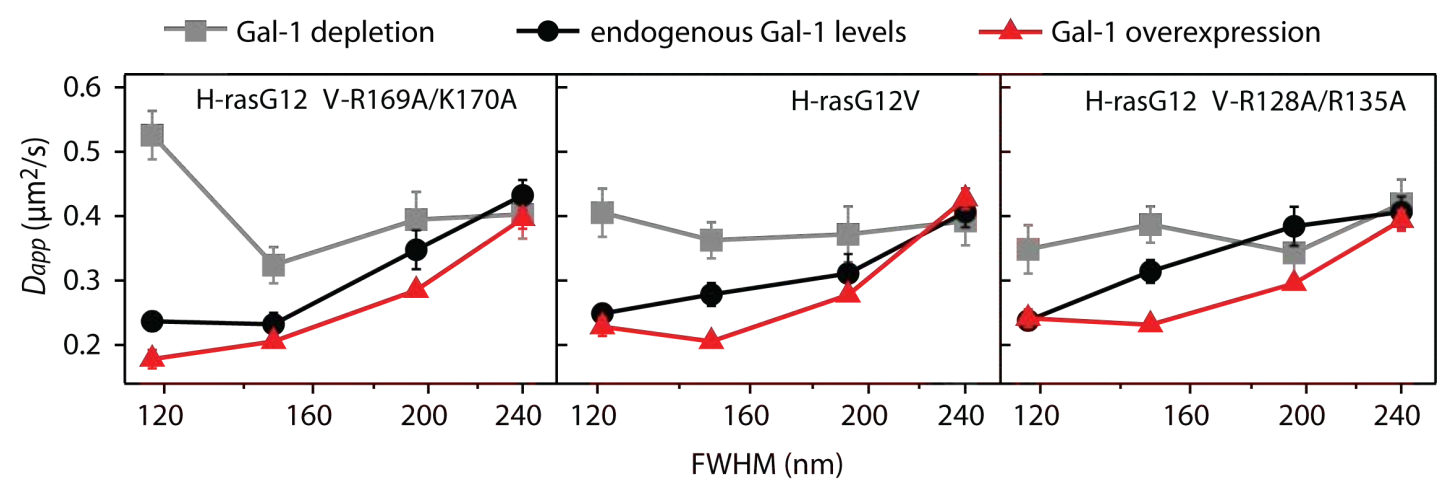

Figure 4.5: Dynamics of three distinct H-Ras orientation-mutants $D_{a p p}(\mathrm{FWHM})$ dependencies for H-Ras orientation-mutants show that galectin-1 (Gal-1) modulates the trapping strength of Ras diffusion. Furthermore, different conformers have different sensitivities to Gal-1 regulation.

\subsection{What is trapping? - Nanodomains and the diffusion law}

STED-FCS is the diffraction-unlimited version of a general approach named spotvariation $\mathrm{FCS}^{53}$. In this approach, the apparent diffusion coefficient obtained through systematic FCS measurements in different observation diameters allows discerning compartmentalized, free and trapping diffusion ${ }^{52,53}$. Trapping diffusion is characterized in this method by a decrease in apparent diffusion coefficient as the observation area is decreased. Intuitively this dependency seems questionable, contrarily to the characteristic diffusion laws for free and compartmentalized diffusion, whose physical intuition seems straightforward. Otherwise stated, one could ask the following important question: How to compare the FCS data provided by a confocal measurement and the collection of data provided by several sub-diffraction FCS measurements? If these two sets of data are in principle different, we would have a clear statistical perception that the experiments carried out at sub-diffraction areas and those carried out at diffraction-limited areas possibly assess information of a different nature, given the boundary conditions of our system. For free diffusion, these two sets of data assess the same information, whereas for compartmentalized diffusion they assess (averaged) diffusion across reflecting barriers in the first case and diffusion within compartments in the second case. From this, their characteristic diffusion laws can be empirically deduced. Henceforth, a possible mathematical visualization of the diffusion law for trapping diffusion is described.

Let $S$ be a two-dimensional heterogeneous surface with area $A$ where identical particles can diffuse. Namely, $S$ has certain regions where the viscosity is $v_{1}$, others where viscosity is $v_{2}$ and so on. Because the diffusing particles are identical, their diffusion 


\section{TRANSIENT TRAPPING OF PLASMA MEMBRANE MOLECULES}

coefficient will depend only on the viscosity of the specific region where they diffuse, acquiring corresponding diffusion coefficients $D_{1}, D_{2}$, and so on. Possible schemes for $S$ are as follows:
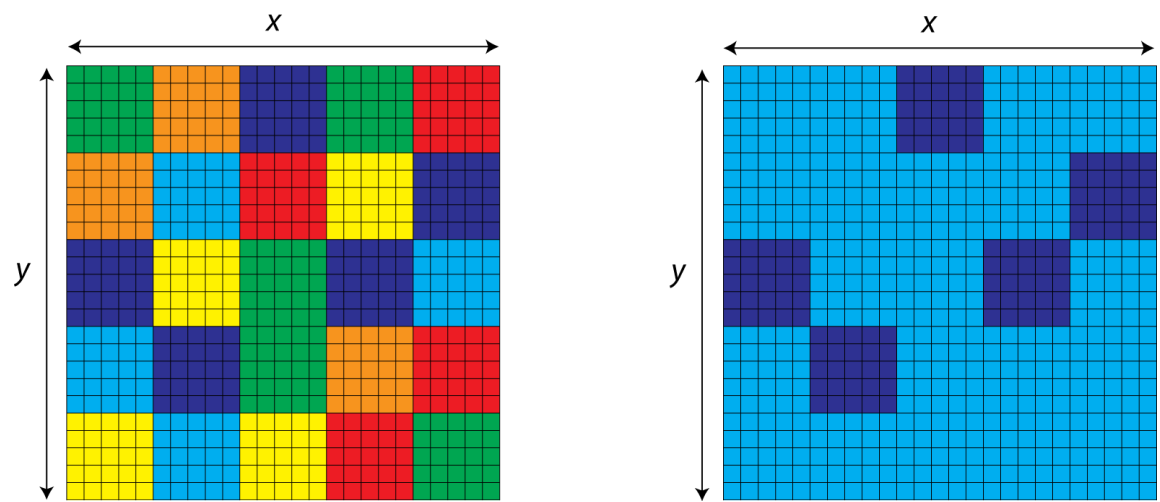

Figure 4.6: Possible partitions for a two-dimensional heterogeneous surface The surface, of total area $A$, is subdivided in small squares of area $a$, where different colors stand for different viscosities.

We assume here that the domains with different viscosities in $S$ are immobile. Let us suppose now that we can perform FCS measurements in areas which are much smaller than the domains, i.e., each one of these small areas has homogeneous viscosity. Let $a$ stand for such small area, such that $A / a=m$. All the correspondent FCS experiments would then only probe free diffusion, though providing different lateral diffusion times, due to different viscosities. The average of the lateral diffusion time over the whole area then would be calculated as

$$
\left\langle\tau_{a}\right\rangle \propto \frac{1}{m} \sum_{x} \sum_{y}\left(\frac{a}{D(x, y)}\right)
$$

where the diffusion coefficient $D$ depends only on the coordinates $\mathrm{x}$, $\mathrm{y}$ of the center of the square being measured. Each measurement corresponds to each small square, which is the step size of this "scanning" procedure. Eq. (4.1) can also be written as

$$
\left\langle\tau_{a}\right\rangle \propto\left\langle\frac{a}{D(x, y)}\right\rangle_{x, y}
$$

where the average over the space was defined as below

$$
\langle f(x, y)\rangle_{x, y} \equiv \frac{1}{m} \sum_{x} \sum_{y} f(x, y)
$$

In order to form a perspective and compare two limiting situations, let us examine now 
the case where the probed area is much larger than the domains. In this case, the FCS experiment "sees" the probed area as a homogeneous surface, not being able to detect small islands of heterogeneity. If we then model this situation as a process where the viscosity, and therefore effective diffusion coefficient through the probed area is being averaged out, we should expect the lateral diffusion time to be

$$
\tau_{A} \propto \frac{A}{\left(\frac{\sum_{x} \sum_{y} D(x, y)}{m}\right)}=\frac{A}{\langle D(x, y)\rangle_{x, y}}=\frac{m a}{\langle D(x, y)\rangle_{x, y}} .
$$

Eqs. (4.2) and (4.4) provide two results for the average transit time through the area $S$, calculated by two different types of experiment. In order to bridge a connection between them, we would like to compare $\tau_{A}$ and $m\left\langle\tau_{a}\right\rangle$. For that purpose, we make use of the Jensen inequality, which can be stated as follows ${ }^{95}$ : if $F(f(\varepsilon))$ is a functional of a function $f(\varepsilon)$, then

$$
\langle F(f(\varepsilon))\rangle_{\varepsilon} \geq F\left(\langle f(\varepsilon)\rangle_{\varepsilon}\right)
$$

if and only if $F$ is a convex functional of $f$ within the interval in which the average is being calculated. Looking at the dependence of the transit diffusion time on the diffusion coefficient, and keeping in mind that the convexity of a curve depends exclusively on the sign of its second derivative, we note that

$$
\frac{\partial^{2}}{\partial D(x, y)^{2}}\left(\frac{1}{D(x, y)}\right)=\frac{2}{D(x, y)^{3}}>0 \forall D(x, y)>0
$$

meaning that the transit time is a convex function of the diffusion coefficient for all possible values of $D(x, y)$. Therefore the inequality (4.5) is valid in any interval, where we identify $F$ as $\tau$ and $f$ as $D$. Hence,

$$
\left\langle\frac{a}{D(x, y)}\right\rangle_{x, y} \geq \frac{a}{\langle D(x, y)\rangle_{x, y}}
$$

from where we have $\tau_{A} \leq m\left\langle\tau_{a}\right\rangle$ and therefore $D_{a p p}(A) \geq\left\langle D_{a p p}(a)\right\rangle$, as indicated by the trapping diffusion law.

Of much importance here is noting that the decrease in the average apparent diffusion coefficient observed in small areas is not a consequence of focusing the probed area on the domains of hindered diffusion. Considering very small domains homogeneously distributed, it would be possible that the percentage of the probed area occupied by the domains is the same for the STED and conventional FCS experiments. What accounts for the difference in the relative transit times and in the shape of the FCS curves is the sensitivity of the experiment to different degrees of heterogeneity. 
4. TRANSIENT TRAPPING OF PLASMA MEMBRANE MOLECULES 


\section{5}

\section{Conclusions and outlook}

In his ingenious "Allegory of the Cave" 96 Plato suggests two sources of delusional perception of reality: artefactual perceived images (stemming from the method of observation) and attribution of substantial meaning and functions to irrelevant effects. This idea finds direct resonance in the problems faced in plasma membrane research, where the dynamic organization and manifold vital functions ascribed to the plasma membrane are sought to be understood.

The investigation of molecular interactions in their native biological environment is fundamentally limited by the method of observation, analogously to the uncertainty principle in quantum mechanics. In this context, the observation of molecular interactions in biological systems may always feature an artefactual component. This limitation can be overcome by observing the same effects by means of different methods. In this way, the genuine character of a phenomenon can be separated from artefactual components. By observing lipid compartmentalized diffusion with a minimally invasive probe via STED-FCS, the research presented in the chapter 3 constitutes the very first validation of the prominent picket fence model for plasma membrane organization proposed by A. Kusumi about a decade ago ${ }^{26,27}$.

This model hypothesizes that lipids and proteins in the plasma membrane are partially segregated into membrane compartments. It has been the subject of intense debate due to the fact that besides high-speed SPT, no other method has until now provided supporting evidence for this hypothesis ${ }^{29}$. Even worse, the SPT experiments reporting lipid compartmentalization employed exclusively artefact-prone lipid probes (gold particles ${ }^{27}$ and quantum dots ${ }^{60}$ ) in order to achieve the required temporal resolution. The artefactual character of these probes stems from the difficulty in validation of their valence towards the target molecule, possibly inducing oligomerization. In the early days of high-speed SPT, this problem was not regarded to be significant ${ }^{97}$, since the Saffman-Delbrück hydrodynamic model predicts that mobility in the plasma membrane has a weak, logarithmic dependence on the radius of the diffusing molecule ${ }^{10}$. A 


\section{CONCLUSIONS AND OUTLOOK}

corollary from the Saffman-Delbrück model is that oligomerization of molecules would only slightly affect their diffusion coefficient. The very significance of that hydrodynamic model was however questioned in 2006, when an experimental study led by Y. Gambin remarkably concluded that diffusion in membranes is not satisfactorily described by the the Saffman-Delbrück equation ${ }^{98}$ and that oligomerization significantly impacts membrane diffusion. These findings cast serious doubt on the results of studies on membrane dynamics which employ polyvalent probes suchs as gold particles and quantum dots, and consequently render unfounded the initial evidence for the picket fence model. Using STED-FCS, we were able to redeem the picket fence model by observing compartmentalized diffusion of lipids with an alternative technique, utilizing fluorescent lipid analogues.

The picket fence model postulates the cortical actin networks to be ultimately responsible for lipid and protein compartmentalized diffusion. These cell networks currently lack well-established proteic markers, such that even the determination of their specific localization within a cell constitutes a challenging task ${ }^{99}$. Interestingly, our pioneer assessment of lipid diffusion upon Arp2/3 modulation pinpoints this protein as a major regulator of cortical actin networks, as compartmentalized diffusion of lipids is specifically suppressed by Arp2/3 inhibition.

Furthermore, lipid diffusion was assessed for the first time in myelin sheets (see chapter 4). Myelin sheets constitute a unique biological membrane which allows the rapid impulse transmission in vertebrates. Having a peculiar lipid composition and being supported by a meshwork of myelin basic protein, this membrane lacks cortical actin networks. In this study, myelin sheets were found to exhibit peculiar diffusion characteristics, where lipids tend to diffuse more freely than in the plasma membrane of the epithelial cell PtK2. As it was shown in chapter 2, the actin cortex in PtK2 cells modulates lipid diffusion through Arp2/3-dependent networks. Importantly, upon extrusion of cortical actin in PtK2 cells (by the introduction of platforms comprising a meshwork of myelin basic protein) lipid diffusion was modulated by this new meshwork instead. This suggests myelin basic protein to be a modulator of lipid diffusion in myelin membrane sheets. Moreover, it corroborates the symbiotic character of the plasma membrane with respect to the proteinaceous sub-membranous structure that defines its morphology.

In another set of experiments, we observed Ras proteins, which are involved in numerous important signalling pathways, and whose nanocluster properties are fundamental to their signalling function. Ras proteins were found to undergo diffusion in the plasma membrane marked by trapping events. This diffusion pattern, consistent with transient nanoclustering, was shown to be regulated by the nanocluster-scaffold protein galectin-1. Furthermore, different conformations of the Ras proteins allowed different susceptibilities to galectin-1 regulation. We assessed diffusion of different conformers of 
Ras in the plasma membrane, and observed specific nanocluster characteristics. From these results, it follows that mutations affecting the conformation of these signalling proteins may also significantly impact their function.

\subsection{Plasma membrane dynamics: perspectives for future research}

Concerning the theoretical basis for the assessment of diffusion by STED-FCS, or spotvariation FCS in general, the development of new theoretical strategies could broaden the discernment of diffusion patterns by this method. In fact, the mathematical analogue of diffusion, i.e. the general problem of random walk, has been intensively studied $^{49}$. A recent study featured mathematically sophisticated implementations of randomly placed barriers, partially inspired by the picket fence model ${ }^{100}$. FCS is the time-domain counterpart of SPT, and is analogous to the time-domain counterpart of the random walk, namely, the classical gambler's ruin problem from game theory ${ }^{101}$. In this classical problem, the expected duration of the game is analogous to the average transit time through the focal spot in FCS experiments. Analytical studies of spotvariation FCS in this background could shed light on potential further applications of this method. In particular, the current spot-variation FCS analysis (based on the diffusion law ${ }^{52}$ ) is analogous to the assessment of the expected duration of the game only. New insights could come from the assessment of the variance of the duration of the game, for example.

A pitfall in plasma membrane research is the premature attribution of pivotal functions to observed effects, which may delay further scientific progress. For example, one reason why the structural model of Danielli and Davson prevailed for such a long time was because it was believed that the monolayer of proteins adsorbed onto both sides of the lipid bilayer was essential to confer the required surface tension to the plasma membrane. The lipid rafts hypothesis may have suffered the same fate, despite being a milestone in plasma membrane research. Countless functions and fundamental implications have been postulated related to these lipid domains, with drug resistance ${ }^{23}$ and increased levels of rafts in cancer cells ${ }^{102}$ being examples. Future research must clarify whether or not these attributions were premature.

The validation of the picket fence model demonstrated in this work raises a new important question, namely, the determination of the precise structural mechanisms by which Arp2/3-dependent cortical actin networks exert compartmentalization in the plasma membrane. The adhesion between the cortical cytoskeleton and the plasma membrane is provided by specific lipids and proteins, in a cell type-dependent manner ${ }^{103}$. Investigation of lipid and protein diffusion upon modulation of membranecytoskeleton adhesion may bring new insights into our understanding of plasma mem- 


\section{CONCLUSIONS AND OUTLOOK}

brane organization.

The currently evolving picture of the plasma membrane will surely undergo many interesting and exciting turns of events in the years to follow, especially concerning the role of membrane dynamics in signal transduction pathways. This is because the first steps of signalling events take place at the plasma membrane, and deciphering their details is fundamental to the understanding of some of the most important questions concerning biological systems.

A highly complex system with intricate membrane-organizing principles, possibly operating symbiotically with cortical actin networks and orchestrating dynamic transient assemblies of lipids and proteins shall emerge as an increasingly more comprehensive concept. 


\section{References}

[1] Gorter, E. And Grendel, F. On bimolecular layers of lipoids on the chromocytes of the blood. The Journal of Experimental Medicine, 41(4):439-443, March 1925.

[2] Luzio, J.P. And Thompson, R.J. Molecular Medical Biochemistry. Cambridge University Press, February 1990.

[3] Danielli, J.F. And Davson, H. A contribution to the theory of permeability of thin films. Journal of Cellular and Comparative Physiology, 5(4):495-508, 1935.

[4] Bernal, J.D. And Crowfoot, D. X-Ray Photographs of Crystalline Pepsin. Nature, 133:794-795, May 1934.

[5] Robertson, J.D. The Membrane of the Living Cell. Scientific American, 206(4):64-72, April 1962.

[6] Singer, S.J. And Nicolson, G.L. The fluid mosaic model of the structure of cell membranes. Science (New York, N.Y.), 175(4023):720-731, February 1972.

[7] Frye, L.D. And Edidin, M. The Rapid Intermixing of Cell Surface Antigens After Formation of Mouse-Human Heterokaryons. Journal of Cell Science, 7(2):319-335, September 1970 .

[8] Pohl, S.L., Birnbaumer, L. and Rodbell, M. The Glucagon-sensitive Adenyl Cyclase System in Plasma Membranes of Rat Liver I. PROPERTIES. Journal of Biological Chemistry, 246(6):1849-1856, March 1971.

[9] Einstein, A. Über die von der molekularkinetischen Theorie der Wärme geforderte Bewegung von in ruhenden Flüssigkeiten suspendierten Teilchen. Annalen der Physik, 17(8):549-560, May 1905.

[10] Saffman, P.G. And DelbrüCK, M. Brownian motion in biological membranes. Proceedings of the National Academy of Sciences, 72(8):3111-3113, August 1975.

[11] Magde, D., Elson, E. And Webb, W.W. Thermodynamic Fluctuations in a Reacting System-Measurement by Fluorescence Correlation Spectroscopy. Physical Review Letters, 29(11):705-708, September 1972.

[12] Axelrod, D., Koppel, D.E., Schlessinger, J., Elson, E. And WebB, W.W. Mobility measurement by analysis of fluorescence photobleaching recovery kinetics. Biophysical Journal, 16(9):1055-1069, September 1976. 
[13] BARAK, L.S. AND WEBB, W.W. Fluorescent low density lipoprotein for observation of dynamics of individual receptor complexes on cultured human fibroblasts. The Journal of Cell Biology, 90(3):595-604, September 1981.

[14] de Brabander, M., Nuydens, R., Ishihara, A., Holifield, B., Jacobson, K. and Geerts, $\mathrm{H}$. Lateral diffusion and retrograde movements of individual cell surface components on single motile cells observed with Nanovid microscopy. The Journal of cell biology, 112(1):111-124, January 1991.

[15] Edidin, M., Kuo, S.C. And Sheetz, M.P. Lateral movements of membrane glycoproteins restricted by dynamic cytoplasmic barriers. Science (New York, N.Y.), 254(5036):1379-1382, November 1991.

[16] Kusumi, A., Sako, Y. and Yамамото, M. Confined lateral diffusion of membrane receptors as studied by single particle tracking (nanovid microscopy). Effects of calciuminduced differentiation in cultured epithelial cells. Biophysical Journal, 65(5):2021-2040, November 1993.

[17] Venkatakrishnan, G., McKinnon, C.A., Pilapil, C.G., Wolf, D.E. and Ross, A.H. Nerve growth factor receptors are preaggregated and immobile on responsive cells. Biochemistry, 30(11):2748-2753, January 1991.

[18] Meer, G.V. Lipid Traffic in Animal Cells. Annual Review of Cell Biology, 5(1):247-275, 1989.

[19] Rodriguez-Boulan, E. And Nelson, W.J. Morphogenesis of the polarized epithelial cell phenotype. Science (New York, N.Y.), 245(4919):718-725, 1989.

[20] Simons, K. And Ikonen, E. Functional rafts in cell membranes. Nature, 387(6633):569572 , June 1997.

[21] HANCOCK, J.F. Lipid rafts: contentious only from simplistic standpoints. Nature Reviews Molecular Cell Biology, 7(6):456-462, June 2006.

[22] Lingwood, D. And Simons, K. Lipid Rafts As a Membrane-Organizing Principle. Science, 327(5961):46-50, January 2010.

[23] Leslie, M. Do Lipid Rafts Exist? Science, 334(6059):1046-1047, November 2011.

[24] Simons, K. and Ehehalt, R. Cholesterol, lipid rafts, and disease. Journal of Clinical Investigation, 110(5):597-603, September 2002.

[25] Munro, S. Lipid Rafts: Elusive or Illusive? Cell, 115(4):377-388, November 2003.

[26] Kusumi, A., Nakada, C., Ritchie, K., Murase, K., Suzuki, K., Murakoshi, H., Kasai, R.S., Kondo, J. And Fujiwara, T. Paradigm shift of the plasma membrane concept from the two-dimensional continuum fluid to the partitioned fluid: high-speed singlemolecule tracking of membrane molecules. Annual Review of Biophysics and Biomolecular Structure, 34(1):351-378, 2005.

[27] Fujiwara, T., Ritchie, K., Murakoshi, H., Jacobson, K. and Kusumi, A. Phospholipids undergo hop diffusion in compartmentalized cell membrane. The Journal of Cell Biology, 157(6):1071-1082, June 2002. 
[28] Kusumi, A., Fujiwara, T.K., Chadda, R., Xie, M., Tsunoyama, T.A., Kalay, Z., Kasai, R.S. And Suzuki, K.G. Dynamic Organizing Principles of the Plasma Membrane that Regulate Signal Transduction: Commemorating the Fortieth Anniversary of Singer and Nicolson's Fluid-Mosaic Model. Annual Review of Cell and Developmental Biology, 28(1):215-250, 2012.

[29] Аввотт, A. Cell biology: Hopping fences. Nature, 433(7027):680-683, February 2005.

[30] Jacobson, K., Sheets, E.D. and Simson, R. Revisiting the fluid mosaic model of membranes. Science, 268(5216):1441-1442, June 1995.

[31] Clausen, M.P. and Lagerholm, B.C. The probe rules in single particle tracking. Current protein $\& 5$ peptide science, 12(8):699-713, December 2011.

[32] Kusumi, A., Shirai, Y.M., Koyama-Honda, I., Suzuki, K.G. and Fujiwara, T.K. Hierarchical organization of the plasma membrane: Investigations by single-molecule tracking vs. fluorescence correlation spectroscopy. FEBS Letters, 584(9):1814-1823, May 2010 .

[33] Gowrishankar, K., Ghosh, S., Saha, S., C., R., Mayor, S. and Rao, M. Active Remodeling of Cortical Actin Regulates Spatiotemporal Organization of Cell Surface Molecules. Cell, 149(6):1353-1367, June 2012.

[34] Born, M. And Wolf, E. Principles of Optics. Cambridge University Press, Cambridge, 2002.

[35] Hell, S.W. and Wichmann, J. Breaking the diffraction resolution limit by stimulated emission: stimulated-emission-depletion fluorescence microscopy. Optics letters, 19(11):780-782, June 1994.

[36] Hell, S.W. Toward fluorescence nanoscopy. Nature Biotechnology, 21(11):1347-1355, November 2003.

[37] HeLl, S.W. Microscopy and its focal switch. Nature Methods, 6(1):24-32, January 2009.

[38] Harke, B., Keller, J., Ullal, C.K., Westphal, V., Schönle, A. And Hell, S.W. Resolution scaling in STED microscopy. Optics Express, 16(6):4154-4162, March 2008.

[39] Hell, S.W. Far-Field Optical Nanoscopy. Science, 316(5828):1153-1158, May 2007.

[40] Willig, K.I., Kellner, R.R., Medda, R., Hein, B., Jakobs, S. and Hell, S.W. Nanoscale resolution in GFP-based microscopy. Nature Methods, 3(9):721-723, September 2006.

[41] Donnert, G., Keller, J., Medda, R., Andrei, M.A., Rizzoli, S.O., Lührmann, R., Jahn, R., Eggeling, C. And Hell, S.W. Macromolecular-scale resolution in biological fluorescence microscopy. Proceedings of the National Academy of Sciences, 103(31):11440-11445, August 2006.

[42] Berning, S., Willig, K.I., Steffens, H., Dibaj, P. and Hell, S.W. Nanoscopy in a Living Mouse Brain. Science, 335(6068):551-551, February 2012.

[43] Göttfert, F., Wurm, C.A., Mueller, V., Berning, S., Cordes, V.C., Honigmann, A. AND Hell, S.W. Coaligned Dual-Channel STED Nanoscopy and Molecular Diffusion Analysis at 20 nm Resolution. Biophysical Journal, 105(1):L01-L03, July 2013. 


\section{REFERENCES}

[44] Eggeling, C. ET AL. Direct observation of the nanoscale dynamics of membrane lipids in a living cell. Nature, 457(7233):1159-1162, February 2009.

[45] Kastrup, L., Blom, H., Eggeling, C. And Hell, S.W. Fluorescence Fluctuation Spectroscopy in Subdiffraction Focal Volumes. Physical Review Letters, 94(17):178104, May 2005.

[46] Ringemann, C., Harke, B., Middendorff, C.v., Medda, R., Honigmann, A., Wagner, R., Leutenegger, M., Schönle, A., Hell, S.W. and Eggeling, C. Exploring singlemolecule dynamics with fluorescence nanoscopy. New Journal of Physics, 11(10):103054, October 2009 .

[47] Mueller, V., Ringemann, C., Honigmann, A., Schwarzmann, G., Medda, R., Leutenegger, M., Polyakova, S., Belov, V., Hell, S. And Eggeling, C. STED Nanoscopy Reveals Molecular Details of Cholesterol- and Cytoskeleton-Modulated Lipid Interactions in Living Cells. Biophysical Journal, 101(7):1651-1660, October 2011.

[48] Mueller, V., Honigmann, A., Ringemann, C., Medda, R., Schwarzmann, G. And Eggeling, C. Chapter One - FCS in STED Microscopy: Studying the Nanoscale of Lipid Membrane Dynamics. In Sergey Y. Tetin, editor, Methods in Enzymology, Volume 519 of Fluorescence Fluctuation Spectroscopy (FFS), Part B, pages 1-38. Academic Press, 2013.

[49] Chandrasekhar, S. Stochastic Problems in Physics and Astronomy. Reviews of Modern Physics, 15(1):1-89, January 1943.

[50] Elson, E.L. And Magde, D. Fluorescence correlation spectroscopy. I. Conceptual basis and theory. Biopolymers, 13(1):1-27, 1974.

[51] Van Orden, A., Fogarty, K. and Jung, J. Fluorescence fluctuation spectroscopy: a coming of age story. Applied spectroscopy, 58(5):122A-137A, May 2004.

[52] Wawrezinieck, L., Rigneault, H., Marguet, D. and Lenne, P.F. Fluorescence Correlation Spectroscopy Diffusion Laws to Probe the Submicron Cell Membrane Organization. Biophysical Journal, 89(6):4029-4042, December 2005.

[53] He, H.T. and Marguet, D. Detecting Nanodomains in Living Cell Membrane by Fluorescence Correlation Spectroscopy. Annual Review of Physical Chemistry, 62(1):417436, 2011.

[54] Murase, K., Fujimara, T., Umemura, Y., Suzuki, K., Iino, R., Yamashita, H., Saito, M., Murakoshi, H., Ritchie, K. And Kusumi, A. Ultrafine Membrane Compartments for Molecular Diffusion as Revealed by Single Molecule Techniques. Biophysical Journal, 86(6):4075-4093, June 2004.

[55] Mennicke, U. and Salditt, T. Preparation of Solid-Supported Lipid Bilayers by SpinCoating. Langmuir, 18(21):8172-8177, October 2002.

[56] Xu, K., Babcock, H.P. And Zhuang, X. Dual-objective STORM reveals threedimensional filament organization in the actin cytoskeleton. Nature Methods, 9(2):185188, February 2012. 
[57] LukinaviČIUs, G. ET AL. A near-infrared fluorophore for live-cell super-resolution microscopy of cellular proteins. Nature Chemistry, 5(2):132-139, February 2013.

[58] Engelman, D.M. Membranes are more mosaic than fluid. Nature, 438(7068):578-580, December 2005.

[59] Kwik, J., Boyle, S., Fooksman, D., Margolis, L., Sheetz, M.P. and Edidin, M. Membrane cholesterol, lateral mobility, and the phosphatidylinositol 4,5-bisphosphatedependent organization of cell actin. Proceedings of the National Academy of Sciences, 100(24):13964-13969, November 2003.

[60] Clausen, M.P. And Lagerholm, B.C. Visualization of Plasma Membrane Compartmentalization by High-Speed Quantum Dot Tracking. Nano Letters, 13(6):2332-2337, June 2013.

[61] Heinemann, F., Vogel, S.K. And Schwille, P. Lateral Membrane Diffusion Modulated by a Minimal Actin Cortex. Biophysical Journal, 104(7):1465-1475, April 2013.

[62] Lillemeier, B.F., Pfeiffer, J.R., Surviladze, Z., Wilson, B.S. And Davis, M.M. Plasma membrane-associated proteins are clustered into islands attached to the cytoskeleton. Proceedings of the National Academy of Sciences, 103(50):18992-18997, December 2006.

[63] SACHS, F. Stretch-Activated Ion Channels: What Are They? Physiology, 25(1):50-56, February 2010.

[64] Wieser, S., Moertelmaier, M., Fuertbauer, E., Stockinger, H. and Schütz, G.J. (Un)Confined Diffusion of CD59 in the Plasma Membrane Determined by HighResolution Single Molecule Microscopy. Biophysical Journal, 92(10):3719-3728, May 2007.

[65] Adler, J., Shevchuk, A.I., Novak, P., Korchev, Y.E. And Parmryd, I. Plasma membrane topography and interpretation of single-particle tracks. Nature Methods, 7(3):170171, March 2010.

[66] Wu, C., Asokan, S.B., Berginski, M.E., Haynes, E.M., Sharpless, N.E., Griffith, J.D., Gomez, S.M. and Bear, J.E. Arp2/3 Is Critical for Lamellipodia and Response to Extracellular Matrix Cues but Is Dispensable for Chemotaxis. Cell, 148(5):973-987, March 2012.

[67] Nolen, B.J., Tomasevic, N., Russell, A., Pierce, D.W., Jia, Z., McCormick, C.D., Hartman, J., Sakowicz, R. And Pollard, T.D. Characterization of two classes of small molecule inhibitors of Arp2/3 complex. Nature, 460(7258):1031-1034, August 2009.

[68] Rotty, J.D., Wu, C. AND BeAR, J.E. New insights into the regulation and cellular functions of the ARP2/3 complex. Nature Reviews Molecular Cell Biology, 14(1):7-12, January 2013.

[69] Goley, E.D. And Welch, M.D. The ARP2/3 complex: an actin nucleator comes of age. Nature Reviews Molecular Cell Biology, 7(10):713-726, October 2006.

[70] Yi, K., Unruh, J.R., Deng, M., Slaughter, B.D., Rubinstein, B. and Li, R. Dynamic maintenance of asymmetric meiotic spindle position through Arp2/3-complex-driven cytoplasmic streaming in mouse oocytes. Nature Cell Biology, 13(10):1252-1258, October 2011. 
[71] Chaigne, A. ET AL. A soft cortex is essential for asymmetric spindle positioning in mouse oocytes. Nature Cell Biology, 15(8):958-966, August 2013.

[72] Zhou, K., Muroyama, A., Underwood, J., Leylek, R., Ray, S., Soderling, S.H. and LECHLER, T. Actin-related protein2/3 complex regulates tight junctions and terminal differentiation to promote epidermal barrier formation. Proceedings of the National Academy of Sciences, page 201308419, September 2013.

[73] Jaqaman, K., Kumata, H., Touret, N., Collins, R., Trimble, W.S., Danuser, G. And Grinstein, S. Cytoskeletal Control of CD36 Diffusion Promotes Its Receptor and Signaling Function. Cell, 146(4):593-606, August 2011.

[74] Suzuki, K.G.N., Fujiwara, T.K., Sanematsu, F., Iino, R., Edidin, M. and Kusumi, A. GPI-anchored receptor clusters transiently recruit Lyn and G $\alpha$ for temporary cluster immobilization and Lyn activation: single-molecule tracking study 1 . The Journal of Cell Biology, 177(4):717-730, May 2007.

[75] Sahl, S.J., Leutenegger, M., Hilbert, M., Hell, S.W. And Eggeling, C. Fast molecular tracking maps nanoscale dynamics of plasma membrane lipids. Proceedings of the National Academy of Sciences, 107(15):6829-6834, April 2010.

[76] Suzuki, K.G.N., Kasai, R.S., Hirosawa, K.M., Nemoto, Y.L., Ishibashi, M., Miwa, Y., Fujiwara, T.K. And Kusumi, A. Transient GPI-anchored protein homodimers are units for raft organization and function. Nature Chemical Biology, 8(9):774-783, September 2012.

[77] Suzuki, K.G.N., Fujiwara, T.K., Edidin, M. and Kusumi, A. Dynamic recruitment of phospholipase $\mathbf{C} \gamma$ at transiently immobilized GPI-anchored receptor clusters induces IP3-Ca2+ signaling: single-molecule tracking study 2. The Journal of Cell Biology, 177(4):731-742, May 2007.

[78] Daumas, F., Destainville, N., Millot, C., Lopez, A., Dean, D. and Salomé, L. Confined Diffusion Without Fences of a G-Protein-Coupled Receptor as Revealed by Single Particle Tracking. Biophysical Journal, 84(1):356-366, January 2003.

[79] Mueller, V.J. Nanoscale studies of membrane dynamics via STED-Fluorescence Correlation Spectroscopy. Dissertation, July 2012.

[80] Chepizhro, O. and Peruani, F. Diffusion, Subdiffusion, and Trapping of Active Particles in Heterogeneous Media. Physical Review Letters, 111(16):160604, October 2013.

[81] Morell, P. and Quarles, R.H. The Myelin Sheath, 1999.

[82] Hartline, D.K. What is myelin? Neuron Glia Biology, 4(02):153-163, 2008.

[83] Baumann, N. and Pham-Dinh, D. Biology of Oligodendrocyte and Myelin in the Mammalian Central Nervous System. Physiological Reviews, 81(2):871-927, April 2001.

[84] Aggarwal, S. et Al. Myelin Membrane Assembly Is Driven by a Phase Transition of Myelin Basic Proteins Into a Cohesive Protein Meshwork. PLoS Biol, 11(6):e1001577, June 2013.

[85] Aggarwal, S. et Al. A Size Barrier Limits Protein Diffusion at the Cell Surface to Generate Lipid-Rich Myelin-Membrane Sheets. Developmental Cell, 21(3):445-456, September 2011. 
[86] Goodsell, D.S. The Molecular Perspective: The ras Oncogene. The Oncologist, 4(3):263-264, June 1999.

[87] Downward, J. Targeting RAS signalling pathways in cancer therapy. Nature Reviews Cancer, 3(1):11-22, January 2003.

[88] Pylayeva-Gupta, Y., Grabocka, E. and Bar-Sagi, D. RAS oncogenes: weaving a tumorigenic web. Nature Reviews Cancer, 11(11):761-774, November 2011.

[89] Vetter, I.R. and Wittinghofer, A. The Guanine Nucleotide-Binding Switch in Three Dimensions. Science, 294(5545):1299-1304, November 2001.

[90] BAKer, N.M. AND Der, C.J. Cancer: Drug for an 'undruggable' protein. Nature, 497(7451):577-578, May 2013.

[91] Janosi, L., Li, Z., Hancock, J.F. And Gorfe, A.A. Organization, dynamics, and segregation of Ras nanoclusters in membrane domains. Proceedings of the National Academy of Sciences, 109(21):8097-8102, May 2012.

[92] Belanis, L., Plowman, S.J., Rotblat, B., Hancock, J.F. and Kloog, Y. Galectin-1 Is a Novel Structural Component and a Major Regulator of H-Ras Nanoclusters. Molecular Biology of the Cell, 19(4):1404-1414, April 2008.

[93] Tian, T., Harding, A., Inder, K., Plowman, S., Parton, R.G. and Hancock, J.F. Plasma membrane nanoswitches generate high-fidelity Ras signal transduction. Nature Cell Biology, 9(8):905-914, August 2007.

[94] Abankwa, D., Gorfe, A.A., Inder, K. and Hancock, J.F. Ras membrane orientation and nanodomain localization generate isoform diversity. Proceedings of the National Academy of Sciences, 107(3):1130-1135, January 2010.

[95] Andrade, D.M. And Hussein, M.S. Jensen inequalities for tunneling probabilities in complex systems. Physical Review C, 80(3):034610, September 2009.

[96] Plato. The Republic. Penguin, London, 2007.

[97] Damjanovich, S., Edidin, M., Szollosi, J. And Tron, L. Mobility and Proximity in Biological Membranes. Taylor \& Francis, March 1994.

[98] Gambin, Y., Lopez-Esparza, R., Reffay, M., Sierecki, E., Gov, N.S., Genest, M., Hodges, R.S. And URBACH, W. Lateral mobility of proteins in liquid membranes revisited. Proceedings of the National Academy of Sciences of the United States of America, 103(7):2098-2102, February 2006.

[99] Salbreux, G., Charras, G. and Paluch, E. Actin cortex mechanics and cellular morphogenesis. Trends in Cell Biology, 22(10):536-545, October 2012.

[100] Novikov, D.S., Fieremans, E., Jensen, J.H. and Helpern, J.A. Random walks with barriers. Nature Physics, 7(6):508-514, June 2011.

[101] FeLleR, W. An introduction to probability theory and its applications. Volume I. J. Wiley \& sons, New York; London; Sydney, 1968. 
[102] Li, Y.C., PARK, M.J., Ye, S.K., Kim, C.W. And Kim, Y.N. Elevated Levels of CholesterolRich Lipid Rafts in Cancer Cells Are Correlated with Apoptosis Sensitivity Induced by Cholesterol-Depleting Agents. The American Journal of Pathology, 168(4):1107-1118, April 2006.

[103] Sheetz, M.P., Sable, J.E. And Döbereiner, H.G. Continuous Membrane-Cytoskeleton Adhesion Requires Continuous Accommodation to Lipid and Cytoskeleton Dynamics. Annual Review of Biophysics and Biomolecular Structure, 35(1):417-434, 2006. 


\section{Appendix A}

\begin{tabular}{|c|c|c|}
\hline & $\begin{array}{l}P \text { value for com- } \\
\text { parison between } \\
(\mathrm{FWHM}=40 \mathrm{~nm}) \\
\text { and }(\mathrm{FWHM}=240 \\
\mathrm{nm}) \text { data in the same } \\
\text { experiment }\end{array}$ & $\begin{array}{l}P \text { value for compari- } \\
\text { son between (FWHM } \\
=240 \mathrm{~nm} \text { ) data and } \\
\text { control experiment } \\
(\mathrm{FWHM}=240 \mathrm{~nm} \\
\text { data) }\end{array}$ \\
\hline Untreated NRK cells (control) & $<0.0001$ & $\mathrm{~N} / \mathrm{A}$ \\
\hline COase-treated NRK cells & $=0.0105$ & $=0.1871$ \\
\hline Blebbistatin-treated NRK cells & $<0.0001$ & $=0.4113$ \\
\hline LatB-treated NRK cells & $=0.0563$ & $<0.0001$ \\
\hline CK-666-treated NRK cells & $=0.8741$ & $<0.0001$ \\
\hline Untreated NRK cells (cell body) & $=0.0002$ & $=0.0224$ \\
\hline
\end{tabular}

Table A.1: List of $P$ values for comparisons between results from STED-FCS experiments in NRK cells, as determined by two-tailed unpaired $t$ test 
A.

\begin{tabular}{|c|c|c|}
\cline { 2 - 3 } \multicolumn{1}{l|}{} & $\begin{array}{l}P \text { value for com- } \\
\text { parison between } \\
(\mathrm{FWHM}=40 \mathrm{~nm}) \\
\text { and }(\mathrm{FWHM}=240 \\
\text { nm) data in the same } \\
\text { experiment }\end{array}$ & $\begin{array}{l}P \text { value for compari- } \\
\text { son between (FWHM } \\
=240 \mathrm{~nm} \text { ) data and } \\
\text { control experiment } \\
(\mathrm{FWHM}=240 \mathrm{~nm} \\
\text { data) }\end{array}$ \\
\hline Untreated IA32 cells (control) & $<0.0001$ & $=0.6046$ \\
\hline COase-treated IA32 cells & $<0.0001$ & $=0.6048$ \\
\hline Blebbistatin-treated IA32 cells & $=0.0005$ & $=0.0210$ \\
\hline LatB-treated IA32 cells & $=0.0582$ & $<0.0001$ \\
\hline CK-666-treated IA32 cells & $=0.4195$ & $=0.0190$ \\
\hline Untreated IA32 2xKD cells & $=0.1486$ & \\
\hline
\end{tabular}

Table A.2: List of $P$ values for comparisons between results from STED-FCS experiments in IA32 cells, as determined by two-tailed unpaired $t$ test 


\section{Acknowledgements}

I would like to thank Prof. Stefan W. Hell for having given me the fantastic opportunity to work in the Nanobiophotonics Department at the Max Planck Institute for Biophysical Chemistry, from whose outstanding scientific environment I have benefited so much. I am also very thankful to him for fully supporting and encouraging my projects throughout the years of my $\mathrm{PhD}$.

I would like to thank Dr. Christian Eggeling for co-supervising my PhD. I am deeply grateful for everything I have learned with this outstanding scientist, scientific-wise or otherwise; to whom I will always be indebted and who will always have my deepest respect.

I am very thankful to Prof. Jörg Enderlein and Prof. Erwin Neher for composing my thesis committee, for being supportive and providing fruitful discussions during the thesis committee meetings.

I thank as well the additional members of the examination board, Prof. Helmut Grubmüller and Prof. Mikael Simons, for showing interest in my work.

I am greatly indebted to all my collaborators, who added so much knowledge and wisdom to my life. Working with these various persons, from various countries and with diverse scientific backgrounds, was certainly one of the things I enjoyed most during my PhD. My special thanks to Mathias Clausen, Olena Steshenko, Camilo Guzmán, Virgínia Borroni, B. Christoffer Lagerholm and Jan Keller-Findeisen, who have changed the way I see science and life in general.

Special thanks also to Marcel Leutenegger, who has shared with me a small part of his great knowledge of so many things, and who was always willing to help.

Thanks to Francisco Balzarotti, for being the best optical table-mate ever.

Thanks to Veronika Mueller and Alf Honigmann, for sharing with me part of their knowledge and experience with STED-FCS, membrane dynamics, general optics, etc, which certainly enriched my research. 
Among the fundamentals to which the Nanobiophotonics group owes its excellence is the outstanding team of engineers and technicians who make unimaginable things possible. I am especially thankful to Tanja Gilat, Ellen Rothermel, Jaydev Jethwa and Marco Roose, for bringing solutions to almost everything.

Thanks to Sebastian Schubert, Nicolai Urban, Christian Eggeling, Olena Steshenko, Jaydev Jethwa and Alf Honigmann for proofreading parts of this thesis.

I am especially indebted to my future husband, Sebastian Schubert, for having supported me unconditionally through the time of my $\mathrm{PhD}$, in all possible ways. Para você, o meu sim.

Finally, I would like to deeply thank my family, especially my parents and grandparents, to whom this thesis is dedicated, for having paved my way for the search of knowledge. 


\section{Curriculum Vitae}

Name:

Address:

Telephone:

Email:

Date and place of birth:

Nationality:

Marital status:

Education

05/2002 - 05/2006

$08 / 2006-09 / 2009$

$10 / 2007-12 / 2007$

Since 07/2010

\section{Fellowships}

$2004-2006$

$2006-2010$

Since $07 / 2010$
Débora Machado Andrade

Zur Hohen Warte 21, 37077 Göttingen, Germany

+49-551-201-2515

dmachad@gwdg.de

22.05.1984, Aracaju, Brazil

Brazilian

single

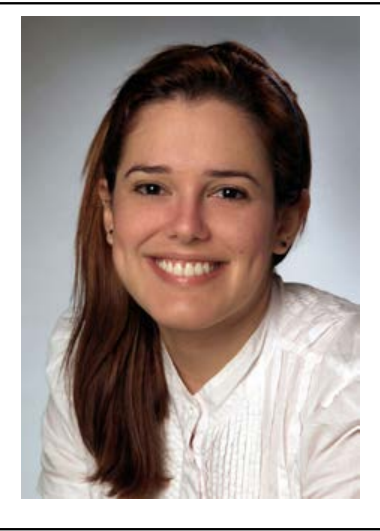

University of Sergipe, São Cristóvão, Sergipe, Brazil

Bachelor of Science in Physics

Bachelor's thesis: "On the two-dimensional Hubbard model in different lattice geometries" Supervisor: Prof. Dr. Cláudio Andrade Macedo

University of São Paulo (USP), São Paulo, Brazil

Master of Science in Physics

Master's thesis: "Jensen's inequality applied to probabilities of nuclear fusion" Supervisor: Prof. Dr. Mahir Saleh Hussein

Max Planck Institute for Physics of Complex Systems (MPI-PKS), Dresden, Germany Research: Effects of Medium on Quantum Tunneling, under the supervision of Prof. Dr. Mahir Saleh Hussein (Martin Gutzwiller Fellow at MPI-PKS in 2007-2008).

International Max Planck Research School for Physics of Biological and Complex Systems of the Max Planck Institute for Biophysical Chemistry (MPIbpc) and the University of Göttingen, Göttingen, Germany

PhD Student at the Department of NanoBiophotonics at the MPIbpc (Prof. Dr. Stefan W. Hell)

\section{Awards}

1999

2009
University of Sergipe, São Cristóvão, Sergipe, Brazil

Undergraduate Research Fellowship of the Institute of Physics

University of São Paulo (USP), São Paulo, Brazil

Graduate Research Fellowship of the Department of Mathematical Physics

Max Planck Institute for Biophysical Chemistry, Göttingen, Germany

PhD Fellowship (from 11/2010 to 10/2012: Max Planck Society IMPRS-PBCS fellowship)

\section{Brazilian National Physics Olympiad for High School Students Gold Medal}

\section{Brazilian Society of Physics}

Poster Prize at Jorge André Swieca Summer School on Theoretical Nuclear Physics for the poster "Jensen inequality applied to the probability of quantum tunneling and to the fusion of heavy ions"

\section{British Biophysical Society}

Poster Prize at Faraday Discussion 161 - Lipids and Membrane Biophysics for the poster

"Lipid compartmentalization at the plasma membrane" 
Débora Machado Andrade

\section{Further Practical Experience}

$09 / 2004-12 / 2004$

University of Sergipe, São Cristóvão, Sergipe, Brazil

Teaching assistant of Prof. Dr. André Maurício Conceição de Souza at the Institute of Physics

Task: Preparing the teaching material and teaching classes to the course "Introduction to Physics" as part of a graduation program on agronomy especially developed by the University of Sergipe for landless workers.

04/2010 - 05/2010 Institute for Research in Biomedicine (IRB), Bellinzona, Switzerland Internship

Task: Supporting cell biology/immunology researchers in conducting experiments.

\section{Other abilities}

Languages

Portuguese (mother tongue), English (fluent in written and spoken; TOEFL IBT: 103 out of 120), Spanish (basics), French (basics), German (basics)

Computer

Fortran (good knowledge), Latex (basics), Microsoft Office (MS Word, MS Excel, MS PowerPoint), Adobe Illustrator (good knowledge)

\section{Publications}

2009

Submitted

In preparation

In preparation
Andrade, D.M.; Hussein, M.S.: Jensen inequalities for tunneling probabilities in complex systems. Phys. Rev. C80 034610, 2009.

Guzmán, C.; Šolman, M.; Ligabue, A.; Blaževitš, O.; Andrade, D.M.; Reymond, L.; Eggeling, C.; Abankwa, D.: H-ras membrane-conformers allosterically couple to differentially Rafrecruitment through nanoclustering.

Andrade, D.M.; Clausen, M.P.; Keller, J.; Mueller, V.; Göttfert, F.; Wu, C.; Bear, J.E.; Hell, S.W.; Lagerholm, B.C.; Eggeling, C.: Lipids are compartmentalized at the plasma membrane by Arp2/3-dependent cortical actin networks.

Steshenko, O.; Andrade, D.M.; Mueller, V.; Hell, S.W.; Eggeling, C.; Simons, M. Myelin-basic protein modulates lipid diffusion in myelin membrane sheets.

\section{Conferences / Workshops}

$01 / 2009$

09/2009

$06 / 2012$

09/2012

$02 / 2013$
Andrade, D.M.; Hussein, M.S.: Jensen inequality applied to the probability of quantum tunneling and to the fusion of heavy ions. Poster at XIV Jorge André Swieca Summer School on Theoretical Nuclear Physics. Itaipava, Brazil

Andrade, D.M.; Hussein, M.S.: Jensen inequalities for tunneling probabilities in complex systems. Poster at the International Workshop and Seminar on Tunneling and Scattering in Complex Systems. Dresden, Germany

Andrade, D.M.; Hell, S.W.; Eggeling, C.: Cell membrane inhomogeneity unraveled by STEDFCS. Oral presentation at $7^{\text {th }}$ Annual Biphysics PhD Meeting. Holbæk, Denmark

Andrade, D.M.; Clausen, M.P.; Lagerholm, C.B.; Hell, S.W.; Eggeling, C.: Lipid compartmentalization at the plasma membrane. Poster at the Faraday Discussion 161 Lipids and Membrane Biophysics. London, England

Andrade, D.M.; Clausen, M.P.; Lagerholm, C.B.; Hell, S.W.; Eggeling, C.: Lipid hop diffusion on the plasma membrane - a STED-FCS investigation. Oral presentation at Biophysical Society $57^{\text {th }}$ Annual Meeting. Philadelphia, United States

Göttingen, November 27th, 2013

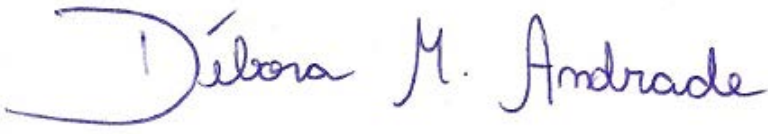

